\author{
Universidade de São Paulo \\ Instituto de Física
}

\title{
Buracos negros cosmológicos
}

\author{
Fabian Ruiz Firavitova
}

Orientador: Prof. Dr. Carlos Molina Mendes

Dissertação de mestrado apresentada ao Instituto de Física da Universidade de São Paulo, como requisito parcial para a obtenção do título de Mestre em Ciências.

Banca Examinadora:

Prof. Dr. Carlos Molina Mendes (EACH/USP)

Prof. Dr. José Ademir Sales de Lima (IAG/USP)

Prof. Dr. Jeferson de Oliveira (UFMT) 


\section{FICHA CATALOGRÁFICA \\ Preparada pelo Serviço de Biblioteca e Informação do Instituto de Física da Universidade de São Paulo}

Ruiz Firavitova, Fabian

Buracos negros cosmológicos. São Paulo, 2018.

Dissertação (Mestrado) - Universidade de São Paulo. Instituto de Física. Depto. de Física Matemática.

Orientador: Prof. Dr. Carlos Molina Mendes.

Área de Concentração: Física.

Unitermos: 1. Relatividade (Física); 2. Gravidade; 3. Cosmologia; 4. Buracos negros. 


\title{
University of São Paulo \\ Physics Institute
}

\section{Cosmological black holes}

\author{
Fabian Ruiz Firavitova
}

Supervisor: Prof. Dr. Carlos Molina Mendes

Dissertation submitted to the Physics Institute of the University of São Paulo in partial fulfillment of the requirements for the degree of Master of Science.

Examining Committee:

Prof. Dr. Carlos Molina Mendes (EACH/USP)

Prof. Dr. José Ademir Sales de Lima (IAG/USP)

Prof. Dr. Jeferson de Oliveira (UFMT) 



\section{Comunión Plenaria}

Los nervios se me adhieren

al barro, a las paredes, abrazan los ramajes, penetran en la tierra, se esparcen por el aire, hasta alcanzar el cielo. El mármol, los caballos tienen mis propias venas. Cualquier dolor lastima mi carne, mi esqueleto.

¡Las veces que me he muerto al ver matar un toro!...

Si diviso una nube debo emprender el vuelo. Si una mujer se acuesta yo me acuesto con ella.

Cuántas veces me he dicho: ¿Seré yo esa piedra? Nunca sigo un cadáver sin quedarme a su lado. Cuando ponen un huevo, yo también cacareo. Basta que alguien me piense para ser un recuerdo.

\section{Oliverio Girondo}





\section{Agradecimentos}

Agradeço profundamente a Mar, quem me motivou a começar essa nova etapa da minha vida aqui no Brasil desde que surgiu a possibilidade quando compartilhamos vidas lá na Venezuela. Quem me acompanhou boa parte do tempo e de quem recebi imponderáveis conselhos. E que ainda hoje, apesar das dificuldades, está ali me apoiando.

A meu amigo Antonio, quem me ajudou demais para chegar ao Brasil, a São Paulo. Pelas ideias trocadas e os conhecimentos que me deixou não apenas da física. Pelos cafés bebidos, pelos cigarros fumados. E sobretudo por aquele voto de confiança que me deu. Agradeço.

A meu amigo Juancito, quem me estendeu a sua mão sem me conhecer quando cheguei, com quem tive inúmeras conversas interessantes de diversas temáticas. Foram muitos bolos integrais e almoços nos finais de semana que compartilhamos. Muitas horas de trabalho e noites sem dormir quando fomos sócios. Agradeço por tudo.

Ao Professor Dr. Carlos Molina Mendes, de quem aprendo em cada reunião, quem teve muita paciência comigo e me ajudou na materialização do presente trabalho. Valoro muito seus conselhos. Agradeço professor.

Finalmente, agradeço ao Conselho Nacional de Desenvolvimento Científico e Tecnológico CNPq pelo financiamento desta pesquisa. 



\section{Resumo}

No contexto da teoria da relatividade geral de Einstein, se estudam espaço-tempos dinâmicos do tipo buraco negro em um ambiente cosmológico, em particular, uma generalização de massa variável do espaço-tempo de McVittie. São apresentados alguns espaço-tempos resultantes de limites da solução de McVittie generalizada. Se discutem as definições padrão de buraco negro e horizonte de eventos assim como as dificuldades de aplicá-las em situações dinâmicas, outras definições para horizontes são exploradas. É usado o conceito de horizonte aparente para localizar buracos negros e horizontes cosmológicos. Soluções para a busca dos horizontes aparentes são encontradas nas geometrias de Schwarzschild-de Sitter, McVittie e McVittie generalizado.

Palavras-chave: buracos negros, cosmologia, geometrias de McVittie generalizadas. 



\section{Abstract}

Black hole-like spacetimes in a cosmological background are studied within the Einstein's general relativity theory framework, in particular a mass varying generalization of the McVittie's spacetime. We exhibit some of the resulting spacetimes as limit cases of the generalized McVittie solution. We discuss the standard definition of black hole and event horizon, and we address the difficulties in aplying it in dynamical situations, another definitions for horizons are explored. The apparent horizon concept is used to locate black holes and cosmological horizons. Solutions for apparent horizons in Schwarzschild-de Sitter, McVittie, and generalized McVittie geometries are shown.

Keywords: black holes, cosmology, generalized McVittie geometries. 



\section{Sumário}

1 Introdução 1

2 Gravidade e relatividade geral $\quad 5$

2.1 Espaço-tempo na relatividade especial . . . . . . . . . . . . . 5

2.2 Espaço-tempo na relatividade geral . . . . . . . . . . . . . . 9 9

2.2 .1 Princípio de equivalência . . . . . . . . . . . . . 9

2.2.2 Princípio de covariância geral . . . . . . . . . . . . 10

2.2.3 Alguns aspectos geométricos . . . . . . . . . . . . . 10

2.2.4 Derivada covariante e curvatura . . . . . . . . . . . . . 16

2.3 Equações de campo . . . . . . . . . . . . . . . . . . . . . . . . . . . . 19

3 Buracos negros e horizontes $\quad 23$

3.1 Espaço-tempo de Schwarzschild e extensão maximal . . . . . . . . . . . . . 25

3.2 Algumas caracterizações para horizontes . . . . . . . . . . . . . . . 31

4 Geometrias de McVittie $\quad 37$

$4.1 \quad$ Limite $a(t) \rightarrow a_{0}, k \rightarrow 0$ (Schwarzschild) . . . . . . . . . . 38

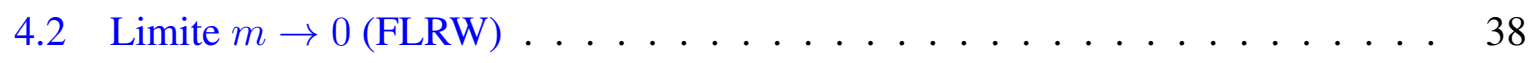

4.2.1 Distâncias e parâmetro de Hubble . . . . . . . . . . . . . . 39

4.2.2 Fluidos perfeitos e as equações de Friedmann . . . . . . . . . . . . . 41

4.3 Métrica de McVittie generalizada . . . . . . . . . . . . . . . . . . . 43

4.3.1 Expansões de congruências geodésicas nulas . . . . . . . . . . . 48

5 Buracos negros cosmológicos $\quad 53$

5.1 Caso $s=0, \sigma=0($ Schwarszchild-de Sitter $) \ldots \ldots \ldots \ldots$

5.2 Casos dinâmicos . . . . . . . . . . . . . . . . . 56

5.2.1 Caso $s=0, \sigma \neq 0($ McVittie $) \ldots \ldots \ldots \ldots$ 
5.2.2 Caso $s \neq 0, \sigma=0$ (McVittie generalizado em fundo de Sitter) . . . . 60

5.2.3 Caso $s \neq 0, \sigma \neq 0($ McVittie generalizado em fundo FLRW) . . . 63

6 Conclusões $\quad 67$

$\begin{array}{ll}\text { A Variedades diferenciais } & 71\end{array}$

B Mapas entre variedades e derivadas de Lie $\quad 77$

B.1 Pullbacks e pushforwards . . . . . . . . . . . . . . 77

B.2 Derivadas de Lie . . . . . . . . . . . . . . . . 79

C McVittie generalizado e as equações de campo de Einstein 81

$\begin{array}{ll}\text { Referências bibliográficas } & 83\end{array}$ 


\section{Capítulo 1}

\section{Introdução}

Depois de 102 anos de serem publicadas as equações de Einstein, e quase 100 anos depois da observação da deflexão da luz proveniente de estrelas distantes pelo campo gravitacional do Sol, [1], ainda surgem novas observações que confirmam a validade da relatividade geral de uma ou outra forma. Entre as mais recentes podemos mencionar as detecções de ondas gravitacionais pelo Laser Interferometer Gravitational-Wave Observatory (LIGO) anunciadas a partir de fevereiro de 2016 [2] e a detecção do redshift gravitacional da luz proveniente da estrela S2 na sua órbita entorno ao buraco negro supermassivo Sagittarius A* no centro da Via Láctea [3]. Cada vez temos mais confiança nas predições da teoria da relatividade de Einstein.

Por outro lado, dentre as teorias que explicam a origem e evolução do universo, temos o modelo cosmológico padrão $\Lambda$-CDM, que apesar de considerado como a melhor descrição da história cósmica, possui elementos que ainda não são muito bem entendidos, como é o caso da matéria e energia escura $[4,5]$. Teorias têm sido construídas para explicar os mecanismos pelos quais essas formas de energia se fixam nos modelos que descrevem a estrutura da matéria. Existe um conjunto recente de propostas que sugerem, que a matéria escura pode estar associada a buracos negros primordiais $[6,7,8]$, que são buracos negros hipotéticos que teriam sido formados em épocas pré-estelares [9]. Surge então a motivação de estudar a formação e evolução de buracos negros em um contexto cosmológico primitivo.

Dentre o conjunto de soluções exatas das equações de Einstein, existem soluções que podem ser interpretadas como espaço-tempos gerados por objetos compactos imersos em fluidos cosmológicos. São generalizações da métrica de Schwarzschild para as equações de Einstein com constante cosmológica diferente de zero. Cabe mencionar a solução de Kottler também 
conhecida como de Schwarzschild-(Anti)de Sitter [10] e a solução de McVittie [11]. A solução de McVittie resolve as equações de Einstein com constante cosmológica e tensor de energia momento de fluido perfeito no exterior de uma inomogeneidade central. Possui simetria esférica e não é assintoticamente plana, além disso, não é estática em geral. A interpretação física de espaço-tempos de McVittie tem sido debatida amplamente desde o momento que surgiu em 1933 [12]. De fato, houve alguma controvérsia na interpretação dessa geometria (ver por exemplo $[13,14,15,16])$. Porém mostrou-se que num espaço cosmológico tipo Einstein-de Sitter [17] a métrica de McVittie se reduz à métrica de Schwarzschild-de Sitter, e o objeto central descrito por tal métrica é um buraco negro.

Existem generalizações dos espaço-tempos de McVittie, como um objeto central carregado [18] e uma massa central dependente do tempo [19]. Foi mostrado que essa última generalização é uma solução exata das equações de Einstein para um campo escalar particular acoplado à gravidade, que pode também ser interpretada como um fluido imperfeito em que um fluxo de calor radial responde pelo aumento na massa do objeto central [20].

Para estabelecer a existência de buracos negros e horizontes cosmológicos na geometria de McVittie generalizada, será necessária uma caracterização dos horizontes em geometrias dinâmicas. A definição geral de buraco negro (uma região do espaço-tempo que não forma parte do passado do infinito futuro nulo do espaço-tempo [21, 22]), precisa do conhecimento da história inteira das geodésicas tipo luz, conhecimento global que nem sempre é garantido quando a geometria é dinâmica. Foram propostas definições mais locais baseadas nas propriedades que esperam-se tenham os horizontes, por exemplo é o caso das trapped surfaces e horizontes aparentes [22, 23, 24, 21], que definem regiões do espaço-tempo nas quais existem famílias de geodésicas nulas que ficam confinadas.

Levando-se em conta os aspectos discutidos sobre a caracterização de buracos negros, abordaremos nas próximas páginas o problema de encontrar horizontes em generalizações da métrica de McVittie, quando a dependência temporal da geometria é controlada por meio de funções associadas com acreção no objeto central e expansão do universo no contexto do modelo cosmológico padrão. Em particular, em [20] se propõe um tipo de função massa e de parâmetro de Hubble que tomamos como referência. Temos interesse nas características originadas na dinâmica e nos seus limites estáticos.

Com tal fim, elaborou-se o seguinte esquema para esta dissertação. No capítulo 2 é apresentado um breve resumo sobre as ideias de espaço e tempo. Comentamos o princípio de equiva- 
lência e de covariância geral que repousam nas bases da teoria da relatividade geral. Expomos a seguir conceitos geométricos que servem para apresentar a linguagem usada. As equações de Einstein na forma usual são introduzidas como as equações de campo da teoria. Se discute também a importância das simetrias na busca de soluções das mesmas.

A continuação, o capítulo 3 foca na solução de Schwarzschild como protótipo. São apresentadas algumas transformações de coordenadas que deixam em evidência a possibilidade de estender o espaço-tempo de Schwarzschild. Se discute a ideia de buraco negro e se introduz a definição usual em termos globais. Outras caracterizações mais geométricas como os horizontes de Killing, trapped surfaces e horizontes aparentes são expostas no final do capítulo.

A geometria de McVittie, a sua generalização com massa variável e os casos limite são abordadas no capítulo 4. É mostrada a vinculação da métrica de McVittie com a expansão cósmica por meio do limite de massa do objeto central nula. Considera-se o papel dos fluidos imperfeitos na descrição dinâmica dos buracos negros cosmológicos. Finalmente são calculadas as expansões de geodésicas nulas em dois sistemas de coordenadas diferentes e é obtida a condição para a existência de horizontes aparentes.

No capítulo 5 apresentam-se soluções para a equação que define os horizontes aparentes, as quais incluem como caso particular as geometrias de Schwarzschild-de Sitter e McVittie. Uma análise qualitativa dos buracos negros cosmológicos obtidos é feita.

Finalmente os apêndices A e B contêm material de apoio para o capítulo 2. Apresenta-se uma definição resumida de variedade diferencial autocontida no apêndice A. No apêndice B mostra-se uma definição para a derivada de Lie baseada na literatura usual [24, 25]. O apêndice C por outro lado, contém as componentes diferentes de zero das equações de Einstein para a métrica de McVittie generalizada, com tensor de energia-momento do fluido imperfeito.

Ao longo desse trabalho o sistema de unidades usado é tal que $G=c=1$, salvo indicação em contrário. Vetores, 1-formas e tensores em geral serão denotados por letras em negrita se quisermos considerar o carácter geral como mapa linear ou multilinear, por exemplo no cálculo da norma de vetores. Porém, a notação com índices será amplamente usada. A tabela 1.1 faz um resumo das convenções na notação de objetos geométricos usadas nesse texto. A tabela 1.2 mostra convenções em sinais que acompanham a notação usada em [42]. 
Tabela 1.1: Alguns exemplos da notação usada nos objetos geométricos que poderia aparecer ao longo do texto.

\begin{tabular}{|c|c|c|c|c|c|}
\hline & $\begin{array}{c}\text { Notação } \\
\text { abstrata }\end{array}$ & $\begin{array}{c}\text { Notação } \\
\text { indexada }\end{array}$ & $\begin{array}{c}\text { Componentes } \\
\text { espaciais }\end{array}$ & $\begin{array}{c}\text { Índice fixo } \\
\text { arbitrário }\end{array}$ & $\begin{array}{c}\text { Índice fixo } \\
\text { particular* }\end{array}$ \\
\hline Vetores & $\boldsymbol{U}, \boldsymbol{V}, \boldsymbol{W}, \boldsymbol{\ell}, \boldsymbol{n}$ & $U^{\mu}, V^{\nu}, W^{\lambda}, \ell^{\alpha}, n^{\beta}$ & $U^{i}, V^{j}, W^{k}, \ell^{m}, n^{l}$ & $U^{\sigma *}, V^{\mu *}, W^{\nu *}$ & $U^{r}, V^{\theta}, W^{t}$ \\
\hline 1-formas & $\boldsymbol{d} f, \boldsymbol{\omega}, d T$ & $\omega_{\mu}, U_{\mu}, V_{\nu}, \ell_{\alpha}, n_{\beta}$ & $\omega_{i}, U_{j}, V_{k}$ & $\omega_{\sigma *}, U_{\mu *}, V_{\nu *}$ & $\omega_{t}, U_{r}, V_{\theta}$ \\
\hline Tensores & $\boldsymbol{T}, \boldsymbol{g}, \boldsymbol{\nabla}$ & $\begin{array}{l}T^{\mu \nu}, g_{\mu \nu}, G^{\alpha}{ }_{\beta}, \nabla_{\sigma} \\
R^{\lambda}{ }_{\mu \sigma \rho}, J_{\alpha}^{\mu}, \delta_{\nu}^{\mu}\end{array}$ & $G^{i}{ }_{j}, T^{j}{ }_{k}$ & $\delta_{\sigma *}^{\mu}$ & $g_{r r}, G^{t}{ }_{t}, T^{t}{ }_{r}$ \\
\hline Objetos \\
não tensoriais
\end{tabular}

Tabela 1.2: Convenções em sinais nas definições de alguns tensores e escalares.

\begin{tabular}{|c|c|}
\hline & Definição \\
\hline $\begin{array}{l}\text { Vetor dirigido } \\
\text { ao futuro }\end{array}$ & $\boldsymbol{V}=\left(V^{0}, V^{1}, V^{2}, V^{3}\right) \operatorname{com} V^{0}>0$ \\
\hline $\begin{array}{l}\text { Norma de } \\
\text { vetores** }\end{array}$ & $+\boldsymbol{g}(\boldsymbol{V}, \boldsymbol{V})=-\left(V^{0}\right)^{2}+\left(V^{1}\right)^{2}+\left(V^{2}\right)^{2}+\left(V^{3}\right)^{2}$ \\
\hline $\begin{array}{l}\text { Tensor de } \\
\text { Riemann }\end{array}$ & $+R_{\sigma \mu \nu}^{\rho}=\partial_{\mu} \Gamma_{\nu \sigma}^{\rho}-\partial_{\nu} \Gamma_{\mu \sigma}^{\rho}+\Gamma_{\mu \lambda}^{\rho} \Gamma_{\nu \sigma}^{\lambda}-\Gamma_{\nu \lambda}^{\rho} \Gamma_{\mu \sigma}^{\lambda}$ \\
\hline $\begin{array}{l}\text { Tensor de } \\
\text { Ricci }\end{array}$ & $+R_{\mu \nu}=R_{\mu \alpha \nu}^{\alpha}$ \\
\hline $\begin{array}{l}\text { Eqs. de } \\
\text { Einstein }\end{array}$ & $G_{\mu \nu}=R_{\mu \nu}-\frac{1}{2} R g_{\mu \nu}=+8 \pi T_{\mu \nu}$ \\
\hline
\end{tabular}




\section{Capítulo 2}

\section{Gravidade e relatividade geral}

\subsection{Espaço-tempo na relatividade especial}

As concepções de espaço e de tempo têm tido muitas modificações ao longo da história [26]. Idealmente e em termos da matemática moderna, para Aristóteles o espaço era euclidiano tridimensional e o tempo um espaço euclidiano unidimensional. Ambas as noções prescindem de uma origem privilegiada por razões de simetria [27]. Nesses espaços euclidianos existe a noção de distância, podendo-se falar de intervalos de separação entre pontos no espaço e no tempo.

Galileu introduziu o agora chamado princípio da relatividade galileana, caracterizado pela invariância de leis dinâmicas sob transformações de coordenadas entre sistemas em movimento relativo uniforme. Não faz sentido falar de um ponto fixo no espaço à medida que o tempo passa. Um observador poderia considerar o ponto fixo e outro observador considerá-o em movimento. Não faz sentido falar de um espaço euclidiano para o tempo todo mas de um espaço euclidiano para cada instante de tempo, um tempo absoluto para todo observador inercial [27].

As leis da mecânica de Newton, junto com a lei de gravitação universal, descreveram com grande sucesso uma variedade de fenômenos observados na natureza. Mas com a formulação posterior das equações de Maxwell para o eletromagnetismo encontrou-se que a luz viaja com a velocidade $c$ independentemente do estado de movimento da fonte e do observador inercial que a mede. Isto sugere que distâncias e intervalos de tempo entre dois eventos sejam diferentes para dois observadores inerciais diferentes $\mathcal{O}$ e $\mathcal{O}^{\prime}$. Se define o intervalo espaço temporal $\mathcal{I}$ 
entre dois eventos como sendo

$$
\mathcal{I}=-c^{2} \Delta t^{2}+\Delta l^{2}
$$

em que $c$ é a velocidade da luz em um sistema de unidades em que $c$ tem dimensões de distância sobre tempo; $\Delta t=t_{2}-t_{1}$ e $\Delta l^{2}=\sum_{i=1}^{3}\left(\Delta \xi^{i}\right)^{2}$ é a separação entre pontos no espaço euclidiano usual em coordenadas cartesianas. Seja um pulso de luz emitido no ponto $P_{1}$ e recebido no ponto $P_{2}$. Os observadores inerciais $\mathcal{O}$ e $\mathcal{O}^{\prime}$ atribuem coordenadas espaço temporais $\left(t_{1}, \xi_{1}^{i}\right),\left(t_{2}, \xi_{2}^{i}\right)$ e $\left(t_{1}^{\prime}, \xi_{1}^{i^{\prime}}\right),\left(t_{2}^{\prime}, \xi_{2}^{i^{\prime}}\right)$. A velocidade da luz é $c$ para ambos e portanto os intervalos espaço temporais $\mathcal{I}$ e $\mathcal{I}^{\prime}$ são iguais, apesar de ser diferentes as medidas de comprimentos e intervalos de tempo de cada observador,

$$
\mathcal{I}=-c^{2} \Delta t^{2}+\Delta l^{2}=0=-c^{2} \Delta t^{\prime 2}+\Delta l^{\prime 2}=\mathcal{I}^{\prime}
$$

Fisicamente, isto implica uma mudança nos conceitos de espaço e tempo, e uma generalização para o intervalo de separação entre dois eventos em dois pontos do espaço-tempo. Aliás, encontrou-se a invariância do intervalo $\mathcal{I}$ para qualquer dois sistemas de coordenadas inercias e qualquer dois eventos. No caso de envolver propagação de luz o intervalo será nulo (2.2).

O intervalo pode ser escrito em termos de uma representação matricial do tensor métrico ou métrica (seção 2.2.3) da relatividade especial, que contém a informação de como combinar as separações espaciais e temporais para obter um intervalo,

$$
\mathcal{I}=\eta_{\mu \nu} \Delta \xi^{\mu} \Delta \xi^{\nu}
$$

Em (2.3) $\mu$ e $\nu$ são índices com valores $0,1,2,3$ de forma que $\xi^{0}=c t$ ( $c$ está em unidades de distância sobre tempo) e $\xi^{1}, \xi^{2}, \xi^{3}$ são coordenadas espaciais. Também foi usada a convenção de Einstein de índices repetidos e será usada ao longo do texto salvo indicação em contrário. Explicitamente em coordenadas cartesianas na convenção de assinatura [24] $(-+++)$ temos,

$$
\eta_{\mu \nu}=\left(\begin{array}{cccc}
-1 & 0 & 0 & 0 \\
0 & 1 & 0 & 0 \\
0 & 0 & 1 & 0 \\
0 & 0 & 0 & 1
\end{array}\right)
$$

As medidas de espaço e tempo de eventos feitas por dois observadores inerciais estão rela- 
cionadas por uma transformação linear como foi mostrado por Einstein em 1905 [28]. Aquelas são as bem conhecidas transformações de Lorentz que podem ser representadas matricialmente com a notação $\Lambda_{\alpha}^{\mu}$. Decorre da invariância do intervalo sob transformações de Lorentz que o tensor métrico satisfaz a relação

$$
\eta_{\alpha \beta}=\eta_{\mu \nu} \Lambda_{\alpha}^{\mu} \Lambda_{\beta}^{\nu}
$$

Na relatividade especial um evento é descrito por um quadrivetor evento $\vec{\xi}=(t, x, y, z)$ cujas componentes $\xi^{\mu}$, em algum sistema de coordenadas, têm a propriedade de transformar da seguinte forma sob uma transformação de Lorentz $\Lambda^{\mu}{ }_{\alpha}$,

$$
\xi^{\mu}=\Lambda_{\alpha}^{\mu} \xi^{\alpha}
$$

Ao longo desse texto será usada uma outra notação útil em alguns casos e que mostra explicitamente a independência das coordenadas em objetos como vetores, matrizes e tensores. Assim, as equações (2.3), (2.5) e (2.6) podem ser expressas em termos da matriz da métrica $\boldsymbol{\eta}$, a matriz de Lorentz $\Lambda$ e o vetor $\boldsymbol{\xi}$, como

$$
\begin{gathered}
\mathcal{I}=\Delta \xi^{T} \eta \Delta \xi \\
\eta=\Lambda^{T} \eta \Lambda \\
\xi^{\prime}=\Lambda \xi
\end{gathered}
$$

Aquelas ideias são parte da justificativa para a construção do espaço de Minkowski $\left(\mathcal{M}_{0}, \boldsymbol{\eta}\right)$. Existe a noção de escalar, no espaço de Minkowski, como aquele invariante sob transformações de Lorentz. O produto escalar de dois quadrivetores fica definido por meio do tensor métrico. Um exemplo de produto escalar está na definição do intervalo espaço temporal (2.3) ou (2.7) que é o produto escalar de $\Delta \xi$ com ele mesmo.

Pode-se falar de curvas no espaço-tempo. Uma forma útil de expressá-las é considerar uma parametrização com um parâmetro $\lambda$ das coordenadas do quadrivetor evento que define a curva. Para cada valor de $\lambda$ em que a curva é bem comportada, está definido o vetor tangente,

$$
V^{\mu}(\lambda)=\frac{d \xi^{\mu}(\lambda)}{d \lambda}
$$

o qual transforma da mesma forma que o quadrivetor evento $\xi^{\mu}$. O produto escalar do vetor 
tangente com ele mesmo pode ser positivo, negativo ou nulo:

$$
\eta_{\mu \nu} V^{\mu} V^{\nu}\left\{\begin{aligned}
>0 & \text { vetor tangente e curva nesse ponto tipo espaço, } \\
<0 & \text { vetor tangente e curva nesse ponto tipo tempo, } \\
=0 & \text { vetor tangente e curva nesse ponto tipo luz ou nulo. }
\end{aligned}\right.
$$

Um escalar importante é o tempo próprio $\tau$, onde $\Delta \tau$, é o intervalo de tempo medido num sistema inercial, onde os eventos inicial e final a ser considerados estão no mesmo ponto espacial do sistema

$$
\Delta \tau=\sqrt{-\mathcal{I}}=\sqrt{-\eta_{\mu \nu} \Delta \xi^{\mu} \Delta \xi^{\nu}}
$$

Suponha-se a existência de eventos $A$ e $B$ unidos por um caminho arbitrário de tipo tempo, parametrizado com o parâmetro $\lambda$. O elemento de linha conectando um par de eventos infinitesimalmente próximos em algum lugar do caminho é expresso com a relação

$$
d s^{2}=\eta_{\mu \nu} d \xi^{\mu} d \xi^{\nu}
$$

em um sistema de coordenadas $\left\{\xi^{\mu}\right\}$. Podemos aplicar uma transformação de Lorentz para um sistema $\left\{\xi^{\prime \mu}\right\}$ em que $d \xi^{\prime i}=0$ para $i=1,2,3$. Assim, $d s^{2}=-\left(d \xi^{\prime 0}\right)^{2}=-d \tau^{2}$. Se tiver um observador no sistema $\left\{\xi^{\prime} \mu\right\}$, ele registraria o tempo próprio $d \tau$ nesse elemento de linha. O procedimento pode ser feito para cada um dos elementos. Logo, pode-se somar os tempos próprios registrados para obter o tempo total ao longo do caminho. Devido à invariância do intervalo sob transformações de Lorentz, temos que o tempo próprio total ao longo da curva vai ser

$$
\Delta \tau=\int \sqrt{-\eta_{\mu \nu} \frac{d \xi^{\mu}}{d \lambda} \frac{d \xi^{\nu}}{d \lambda}} d \lambda
$$

Se o caminho for tipo espaço não existe um observador capaz de acompanhar o caminho e também não existe o tempo próprio. Mas o comprimento do caminho pode ser calculado com uma relação similar

$$
\Delta s=\int \sqrt{\eta_{\mu \nu} \frac{d \xi^{\mu}}{d \sigma} \frac{d \xi^{\nu}}{d \sigma}} d \sigma
$$

Sendo o parâmetro $\sigma$ algum parâmetro apropriado diferente de $\lambda$. No caso de curvas de tipo luz, o comprimento total do caminho vai ser nulo. 


\subsection{Espaço-tempo na relatividade geral}

\subsubsection{Princípio de equivalência}

Uma nova concepção sobre o espaço e o tempo surgiu com o desenvolvimento da relatividade geral ao estudar e generalizar o princípio de equivalência fraco (ou de Galileu), o qual estabelecia inicialmente (através da experiência) a universalidade da queda livre de partículas materiais em um campo gravitacional, ou seja, que toda partícula material em um campo gravitacional uniforme e estático, sente a mesma aceleração devido a gravidade independente de sua estrutura e composição [29]. Matematicamente, isto implica a igualdade entre as massas gravitacional $\left(m_{g}\right)$ e inercial $\left(m_{i}\right)$ :

$$
\vec{a}=-\frac{m_{g}}{m_{i}} \nabla \phi=-\nabla \phi
$$

Assim, uma experiência cinemática (não gravitacional) em uma caixa selada, em queda livre em relação a uma fonte de campo gravitacional uniforme e estático, ou com velocidade uniforme afastado da influência de campos gravitacionais, são equivalentes. A afirmação anterior ainda será válida quando a caixa for pequena o suficiente em comparação com eventuais variações na uniformidade do campo gravitacional e quando as experiencias mecânicas acontecessem em intervalos de tempos curtos o suficiente em comparação com eventuais variações temporais do campo gravitacional.

A generalização chamada princípio de equivalência de Einstein [29], além da equivalência entre experiências mecânicas clássicas (nas condições expressas acima) com ou sem gravitação, precisa que as leis da física sejam localmente invariantes de Poincaré (grupo de Lorentz restrito mais grupo de traslações), ou seja, que localmente se satisfaz a relatividade restrita.

Na sua forma forte, pode ser estabelecido da forma seguinte: através de uma escolha apropriada de sistema (sistema em queda livre), as leis da física descrevem-se localmente da mesma forma que na relatividade restrita [30]. O princípio sugere que os efeitos da gravidade podem ser descritos matematicamente por uma mudança do tensor métrico [31], e podem ser anulados localmente. 


\subsubsection{Princípio de covariância geral}

Em relatividade geral assume-se que o princípio de equivalência de Einstein na sua forma forte é válido e o campo gravitacional é representado por um tensor métrico no espaço-tempo, de forma que sua ação é igual para toda partícula. O que diz o princípio de covariância geral é que, em virtude do princípio de equivalência de Einstein, uma equação física vai se manter em um campo gravitacional arbitrário, [21] se:

1. A forma da equação mantém-se na ausência de gravidade, ou seja, quando o tensor métrico é o de Minkowski.

2. A equação é covariante, ou seja, preserva sua forma sob uma transformação geral de coordenadas.

O espaço-tempo da relatividade geral é descrito por uma variedade diferenciável (ver apêndice A) $\mathcal{M}$ de 4 dimensões junto com um tensor métrico $\boldsymbol{g}$, e uma conexão $\boldsymbol{\nabla}$ simétrica e compatível com o tensor métrico (será abordado na próxima seção) [27, 25]. Decorre de aqui que cada região do espaço-tempo pode ser descrita em $\mathbb{R}^{4}$ por meio de uma carta (sistema de coordenadas sobre um conjunto aberto em $\mathbb{R}^{n}$ ) e duas de estas regiões que se sobrepõem devem ter uma relação suave entre elas [24].

\subsubsection{Alguns aspectos geométricos}

É útil pensar nos vetores ou campos vetoriais de uma maneira independente das coordenadas. Recorremos portanto ao conceito de espaço tangente ao ponto $p \in \mathcal{M}$, um espaço vetorial denotado $T_{p}$ [24]. Consideremos o espaço $\mathcal{F}$ de mapas de $\mathscr{C}^{\infty}$, por exemplo, $f: \mathcal{M} \rightarrow \mathbb{R}$. Cada curva passando pelo ponto $p$ define um operador derivada direcional em $p, d f / d \lambda$. O espaço tangente pode ser identificado com o espaço de operadores derivada direcional ao longo de curvas que passam por $p$. É um espaço vetorial que tem a mesma dimensão de $\mathcal{M}$. Ao invocar uma carta cada derivada direcional pode ser expressa em termos de derivadas ao longo de cada $x^{\mu}$

$$
\frac{d f}{d \lambda}=\frac{d x^{\mu}}{d \lambda} \frac{\partial f(\boldsymbol{x})}{\partial x^{\mu}}
$$

o que é válido para todo mapa $f$ ao menos uma vez diferenciável. Logo

$$
\frac{d}{d \lambda}=\frac{d x^{\mu}}{d \lambda} \partial_{\mu}=V^{\mu} \partial_{\mu}=V^{\mu} \hat{e}_{\mu}
$$


As componentes do vetor são as componentes do vetor tangente à curva que define o operador derivada direcional. Na carta escolhida, $\partial_{\mu}$ representa uma base para o espaço tangente. Esta definição é independente das coordenadas. Assim, um vetor $\boldsymbol{V}$ pode ser escrito em uma outra base

$$
\boldsymbol{V}=V^{\mu} \partial_{\mu}=V^{\mu^{\prime}} \partial_{\mu^{\prime}}=V^{\mu^{\prime}} \frac{\partial x^{\mu}}{\partial x^{\mu^{\prime}}} \partial_{\mu}
$$

em que se fez uso da regra da cadeia. Segue que as componentes dos vetores transformam sob uma mudança de carta como

$$
V^{\mu^{\prime}}=\frac{\partial x^{\mu^{\prime}}}{\partial x^{\mu}} V^{\mu}
$$

que é compatível com a transformação de Lorentz usual.

Pode-se definir também um campo vetorial que atribui um vetor para cada um dos pontos da variedade, tomando funções suaves e retornando funções suaves. Por outro lado, se define o comutador de dois campos vetoriais $\boldsymbol{X}$ e $\boldsymbol{Y}$ por meio de sua ação sobre um mapa $f$ como

$$
[\boldsymbol{X}, \boldsymbol{Y}](f)=\boldsymbol{X}(\boldsymbol{Y}(f))-\boldsymbol{Y}(\boldsymbol{X}(f))
$$

sendo de novo um campo vetorial que satisfaz as seguintes propriedades:

- $[\boldsymbol{X}, \boldsymbol{Y}](a f+b g)=a[\boldsymbol{X}, \boldsymbol{Y}](f)+b[\boldsymbol{X}, \boldsymbol{Y}](g), \quad$ (linearidade)

- $[\boldsymbol{X}, \boldsymbol{Y}](f g)=f[\boldsymbol{X}, \boldsymbol{Y}](g)+g[\boldsymbol{X}, \boldsymbol{Y}](f), \quad$ (regra de Leibniz)

- $[\boldsymbol{X}, \boldsymbol{Y}]^{\mu}=X^{\nu} \partial_{\nu} Y^{\mu}-Y^{\nu} \partial_{\nu} X^{\mu}$.

As 1-formas definidas como elementos de um espaço dual a $T_{p}$ denotado $T_{p}^{*}$ vão ser o conjunto de mapas lineares $\boldsymbol{\omega}: T_{p} \rightarrow \mathbb{R}$ também chamados vetores duais. Recorremos agora ao conceito de gradiente e de derivada direcional. Para uma escolha arbitrária de carta temos

$$
d f=\partial_{\mu} f d x^{\mu}
$$

que já é um objeto invariante sob transformações de coordenadas. Ao contrário de $f, d f$ existe apenas num ponto (na mesma forma que os vetores) e não dependem da informação em outros pontos de $\mathcal{M}$. É útil pensar em objetos do tipo de $d f$ como vetores duais. Neste caso expressado 
em uma base $d x^{\mu}$, e a sua ação sobre um vetor como a derivada direcional de $f$,

$$
\boldsymbol{d} f\left(\frac{d}{d \lambda}\right)=\partial_{\mu} f d x^{\mu}\left(\frac{d x^{\nu}}{d \lambda} \partial_{\nu}\right)=\partial_{\mu} f \frac{d x^{\nu}}{d \lambda} d x^{\mu}\left(\partial_{\nu}\right)=\frac{d f}{d \lambda}
$$

em que se define a operação $d x^{\mu}\left(\partial_{\nu}\right)=\delta_{\nu}^{\mu}$, onde $\delta_{\nu}^{\mu}$ é o símbolo de Kronecker invariante sob transformações de carta que vale 1 se $\mu=\nu$ e 0 se $\mu \neq \nu$. Logo, $d x^{\mu}$ é uma base apropriada nas coordenadas $\left\{x^{\mu}\right\}$ para o espaço $T_{p}^{*}$. Para transformar 1-formas assume-se que elas são independentes da escolha da carta,

$$
\boldsymbol{\omega}=\omega_{\mu} d x^{\mu}=\omega_{\mu^{\prime}} d x^{\mu^{\prime}}=\omega_{\mu^{\prime}} \frac{\partial x^{\mu^{\prime}}}{\partial x^{\mu}} d x^{\mu}
$$

em que se fez uso da regra da cadeia. Decorre que as componentes das 1-formas transformam sob uma mudança de carta, assim

$$
\omega_{\mu^{\prime}}=\frac{\partial x^{\mu}}{\partial x^{\mu^{\prime}}} \omega_{\mu}
$$

Um tensor $(k, l)$ pode ser entendido como um mapa multilinear que toma $k 1$-formas e $l$ vetores e os leva para $\mathbb{R}[24]$,

$$
\boldsymbol{T}: \underbrace{T_{p}^{*} \times \ldots \times T_{p}^{*}}_{k-\text { vezes }} \times \underbrace{T_{p} \times \ldots \times T_{p}}_{\text {l-vezes }} \rightarrow \mathbb{R} .
$$

As suas componentes em uma base de coordenadas podem ser obtidas aplicando o tensor sobre bases de 1-formas e vetores conforme

$$
T_{\nu_{1}, \ldots, \nu_{l}}^{\mu_{1}, \ldots, \mu_{k}}=\boldsymbol{T}\left(d x^{\mu_{1}}, \ldots, d x^{\mu_{k}}, \partial_{\nu_{1}}, \ldots, \partial_{\nu_{l}}\right) .
$$

Assim, um tensor expandido em uma base (embora não seja o mais natural quando opera-se com eles) será

$$
\boldsymbol{T}=T^{\mu_{1}, \ldots, \mu_{k}}{ }_{\nu_{1}, \ldots, \nu_{l}} \partial_{\mu_{1}} \otimes \ldots \otimes \partial_{\mu_{k}} \otimes d x^{\nu_{1}} \otimes \ldots \otimes d x^{\nu_{l}}
$$

Com um argumento igual que no caso de vetores e 1-formas segue-se que as componentes dos tensores transformam sob uma mudança de carta, assim

$$
T_{1, \ldots, \mu_{k}^{\prime}}^{\mu_{\nu}^{\prime}, \ldots, \nu_{l}^{\prime}}=\frac{\partial x^{\mu_{1}^{\prime}}}{\partial x^{\mu_{1}}} \cdots \frac{\partial x_{k}^{\mu_{k}^{\prime}}}{\partial x^{\mu_{k}}} \frac{\partial x^{\nu_{1}}}{\partial x_{1}^{\nu_{1}^{\prime}}} \cdots \frac{\partial x^{\nu_{l}}}{\partial x^{\nu_{l}^{\prime}}} T^{\mu_{1}, \ldots, \mu_{k}}{ }_{\nu_{1}, \ldots, \nu_{l}}
$$


Um tensor tipo $(k, l)$ pode agir sobre um conjunto de $q$ 1-formas e $p$ vetores com $q \leq k$ e $p \leq l$, definindo assim um novo tensor tipo $(k-q, l-p)$. No caso em que $q=k$ e $p=l$, obtémse um escalar. Também, um tensor tipo $(k, l)$ pode agir sobre um tensor tipo $(m, n)$ tornando-se um tensor $(k-n, l-m)$ se for $k \geq n$ e $l \geq m$, um tensor $(m-l, n-k)$ se for $k<n$ e $l<m$, um tensor $(0, n-k+l-m)$ se for $k<n$ e $l \geq m$ e um tensor $(k-n+m-l, 0)$ se for $k \geq n$ e $l<m$. Aliás, existem múltiplas formas na escolha das entradas do tensor. E novamente, no caso em que $l=m$ e $k=n$ o resultado será um escalar. Combinação das operações anteriores é uma outra possibilidade.

Um tensor que vai ser importante no desenvolvimento deste trabalho é o tensor métrico $\boldsymbol{g}$, normalmente denotado em componentes $g_{\mu \nu}$ e dotado com certas propriedades:

- É um tensor simétrico, ou seja $g_{\mu \nu}=g_{\nu \mu}$.

- Tem determinante não nulo, $g=\left|g_{\mu \nu}\right| \neq 0$.

Desse modo pode-se garantir a existência da sua inversa $\boldsymbol{g}^{-1}$, normalmente denotada em componentes $g^{\mu \nu}$ tal que $g^{\mu \nu} g_{\nu \sigma}=\delta_{\sigma}^{\mu}$. A simetria da métrica vai implicar a simetria da sua inversa [21].

O tensor métrico em relatividade geral está associado com uma série de conceitos e ideias. Por exemplo, define o produto escalar entre vetores e portanto a norma, é usado para construir uma classe de conexões $\nabla$ compatíveis com o tensor métrico que por vez define a derivada covariante permitindo calcular geodésicas. Em particular, geodésicas tipo luz definem em cada ponto da variedade cones de luz que dão a noção de passado e futuro localmente.

Os campos gravitacionais afetam a forma em que se medem comprimentos e tempos, efeitos que podem ser levados em conta com um tensor métrico. O tensor métrico será o campo apropriado na generalização do potencial gravitacional newtoniano e os sistemas de referência em queda livre devem ter tensores métricos de Minkowski, ao menos localmente.

O produto interno de dois vetores é construído usando o tensor métrico ou métrica da forma seguinte

$$
\begin{aligned}
\boldsymbol{V} \cdot \boldsymbol{W}=\boldsymbol{g}(\boldsymbol{V}, \boldsymbol{W}) & =\left(g_{\mu \nu} d x^{\mu} \otimes d x^{\nu}\right)\left(V^{\sigma} \partial_{\sigma}, W^{\rho} \partial_{\rho}\right) \\
& =g_{\mu \nu} V^{\sigma} W^{\rho}\left(d x^{\mu} \otimes d x^{\nu}\right)\left(\partial_{\sigma}, \partial_{\rho}\right) \\
& =g_{\mu \nu} V^{\mu} W^{\nu}
\end{aligned}
$$

em que $\boldsymbol{g}(\boldsymbol{V}, \boldsymbol{W})$ é a ação do tensor $\boldsymbol{g}$ sobre os vetores $\boldsymbol{V}$ e $\boldsymbol{W}$. Existe a possibilidade do 
tensor métrico $\boldsymbol{g}$ agir apenas sobre um vetor $\boldsymbol{V}$ (de componentes $V^{\mu}$ ), o que gera um vetor dual geralmente denotado em componentes como $V_{\mu}$. Similarmente $\boldsymbol{g}^{-1}$ agindo sobre uma 1forma $\boldsymbol{\omega}$ (de componentes $\omega_{\mu}$ ) gera um vetor geralmente denotado em componentes $\omega^{\mu}$. Dessa forma se definem as operações para subir e baixar índices sobre vetores e 1-formas e pode ser estendido a tensores arbitrários.

O produto escalar de um vetor tangente com ele mesmo também pode ser negativo, positivo ou nulo, da mesma forma que na seção 2.1 para curvas no espaço de Minkowski, definindo assim nesse ponto se a curva e o vetor tangente são tipo tempo, tipo espaço ou tipo luz.

A forma canônica do tensor métrico está dada por uma matriz diagonal com entradas $+1,-1,0$. Se algum autovalor for nulo, o determinante do tensor métrico também será nulo e a inversa dele não estará definida. Se tiver vários elementos com sinal menos se diz pseudo-riemanniano. Se tiver só um elemento com sinal menos também se diz lorentziano. O espaço-tempo da relatividade geral tem tensores métricos lorentzianos.

Sempre é possível escrever o tensor métrico na sua forma canônica ao menos em um ponto, mas não necessariamente na sua vizinhança [24]. Resulta que em qualquer ponto $p \in \mathcal{M}$ existe um sistema de coordenadas $\left\{x^{\hat{\mu}}\right\}$ no qual $g_{\hat{\mu} \hat{\nu}}$ se escreve na sua forma canônica de forma que as suas primeras derivadas são nulas $\partial_{\hat{\sigma}} g_{\hat{\mu} \hat{\nu}}=0$ enquanto suas derivadas segundas não (ver por exemplo [21, 24]), em primeira ordem,

$$
g_{\hat{\mu} \hat{\nu}}=\eta_{\hat{\mu} \hat{\nu}}
$$

As coordenadas $\left\{x^{\hat{\mu}}\right\}$ são chamadas coordenadas localmente inerciais. A base associada constitui um sistema local de Lorentz. Isto formaliza a ideia de que pequenas regiões do espaço-tempo têm aparência plana.

Um aspecto que pode ser considerado é o uso do princípio de covariância geral para generalizar equações conhecidas do espaço plano a espaços mais gerais. A equação de movimento de partícula livre massiva em coordenadas cartesianas $\left\{\xi^{\alpha}\right\}$ no espaço de Minkowski, $d^{2} \xi^{\alpha} / d \tau^{2}=0$ pode-se escrever em um sistema de coordenadas geral $\left\{x^{\mu}\right\}$, ao usar o jacobiano da transformação $J_{\mu}^{\alpha}=\partial \xi^{\alpha} / \partial x^{\mu}$ assumido como invertível

$$
\frac{d^{2} \xi^{\alpha}}{d \tau^{2}}=\frac{\partial \xi^{\alpha}}{\partial x^{\mu}}\left[\frac{d^{2} x^{\mu}}{d \tau^{2}}+\frac{\partial x^{\mu}}{\partial \xi^{\beta}} \frac{\partial^{2} \xi^{\beta}}{\partial x^{\nu} \partial x^{\lambda}} \frac{d x^{\nu}}{d \tau} \frac{d x^{\lambda}}{d \tau}\right]=0
$$


Logo a equação de uma linha reta nas novas coordenadas vai ser o que acompanha à matriz jacobiana igualado a zero. No segundo termo de (2.33) aparece um fator que faz a diferença real entre a equação nas coordenadas gerais e nas coordenadas cartesianas. Fisicamente esse termo contém informação sobre pseudo-forças que aparecem nas novas coordenadas. Se define (não é a definição mais rigorosa),

$$
\Gamma_{\nu \lambda}^{\mu}=\frac{\partial x^{\mu}}{\partial \xi^{\beta}} \frac{\partial^{2} \xi^{\beta}}{\partial x^{\nu} \partial x^{\lambda}}
$$

como os símbolos de Christoffel. Pode-se observar que o jacobiano de uma transformação de Lorentz não depende das coordenadas. Logo, os símbolos de Christoffel são todos nulos para esse caso. Decorre, usando o princípio de covariância geral, seção 2.2.2, que a equação adequada para uma partícula massiva em um campo gravitacional arbitrário em qualquer sistema de coordenadas, vai ser,

$$
\frac{d^{2} x^{\mu}}{d \tau^{2}}+\Gamma_{\nu \lambda}^{\mu} \frac{d x^{\nu}}{d \tau} \frac{d x^{\lambda}}{d \tau}=0
$$

A equação (2.35) é conhecida como equação geodésica para o caso em que a partícula for massiva. Existe uma relação entre o jacobiano de uma transformação de coordenadas $\left\{\xi^{\alpha}\right\} \rightarrow$ $\left\{x^{\mu}\right\}$ e o tensor métrico [21],

$$
\partial_{\lambda} g_{\mu \nu}=\eta_{\alpha \beta}\left(J_{\mu}^{\alpha} \partial_{\lambda} J_{\nu}^{\beta}+J_{\nu}^{\beta} \partial_{\lambda} J_{\mu}^{\alpha}\right)
$$

em que o simbolo $\partial_{\lambda}$ denota derivação respeito de $x^{\lambda}$. Com isto, pode-se mostrar que os símbolos de Christoffel podem ser escritos como

$$
\Gamma_{\nu \lambda}^{\mu}=\frac{1}{2} g^{\mu \rho}\left(\partial_{\lambda} g_{\rho \nu}+\partial_{\nu} g_{\rho \lambda}-\partial_{\rho} g_{\nu \lambda}\right)
$$

a qual torna-se a definição usual dos símbolos de Christoffel. Também é importante levar em conta que $\Gamma_{\mu \nu}^{\lambda}$ não são tensores. Sob uma transformação de coordenadas eles satisfazem uma lei de transformação dada por

$$
\Gamma_{\nu \rho}^{\mu}=J_{\alpha}^{\mu} J_{\nu}^{\beta} J_{\rho}^{\gamma} \Gamma_{\beta \gamma}^{\alpha}+J_{\alpha}^{\mu} \partial_{\rho} J_{\nu}^{\alpha}
$$

Uma outra forma de definir os símbolos de Christoffel parte da definição de derivada covariante (na próxima seção será abordada), um objeto que generaliza a derivada parcial usual mas 
preserva sua forma sob transformações locais de coordenadas. Na construção da derivada covariante segundo [24], a covariância requer um termo somado à derivada parcial. Esse termo adicional é proporcional a um objeto com três índices, são os chamados símbolos da conexão. Na referência [24] são denotados como $\widehat{\Gamma}_{\nu \lambda}^{\mu}$ e transformam segundo a equação (2.38). Em geral não estão definidos por (2.37).

Cada derivada covariante se caracteriza pelos seus símbolos da conexão. Os símbolos de Christoffel são os símbolos da chamada conexão afim $\Gamma_{\nu \lambda}^{\mu}$. Eles são simétricos em $\nu$ e $\lambda$. De forma que uma troca de índices não define uma nova derivada covariante. A diferença $\Gamma_{\nu \lambda}^{\mu}-\Gamma_{\lambda \nu}^{\mu}=0$.

No entanto os símbolos de uma conexão qualquer não necessariamente são simétricos. Pode se mostrar com a equação de transformação dos símbolos da conexão (2.38) que a diferença entre símbolos de conexões diferentes $\widehat{\Gamma}_{\nu \lambda}^{\mu}$ e $\tilde{\Gamma}_{\nu \lambda}^{\mu}$ é um tensor. Quando $\tilde{\Gamma}_{\nu \lambda}^{\mu}$ for simplesmente o resultado de trocar índices inferiores na conexão (escolhida arbitrariamente) $\widehat{\Gamma}_{\nu \lambda}^{\mu}$, a diferença entre símbolos será um tensor antissimétrico chamado tensor de torção

$$
T_{\nu \lambda}^{\mu}=\widehat{\Gamma}_{\nu \lambda}^{\mu}-\widehat{\Gamma}_{\lambda \nu}^{\mu}
$$

Se observa que a conexão definida por meio dos símbolos de Christoffel tem torção nula por ter símbolos simétricos. A relatividade geral de Einstein em que está baseada esse trabalho usa a conexão afim na sua geometria.

\subsubsection{Derivada covariante e curvatura}

A derivada ordinária $\partial_{\mu}$ depende do sistema de coordenadas usado. Considere-se uma transformação geral de coordenadas do sistema $\left\{y^{\alpha}\right\}$ para $\left\{x^{\mu}\right\}$ [21] e a consequente mudança na derivada ordinária de um vetor

$$
\partial_{\alpha} V^{\beta}=J_{\alpha}^{\mu} \partial_{\mu}\left(J_{\nu}^{\beta} V^{\nu}\right)
$$

Um pouco de manipulação algébrica e o uso da relação (2.38) levam a

$$
\partial_{\alpha} V^{\beta}=J_{\alpha}^{\mu} J_{\nu}^{\beta}\left(\partial_{\mu} V^{\nu}+\Gamma_{\mu \lambda}^{\nu} V^{\lambda}\right)-\Gamma_{\alpha \gamma}^{\beta} J_{\nu}^{\gamma} V^{\nu}
$$


onde aparece no lado direito um termo com símbolos de Christoffel no sistema de partida $\left\{y^{\alpha}\right\}$. Esse termo colocado no lado esquerdo consegue estabelecer uma estrutura que não muda de forma sob transformações de coordenadas. Logo, se define aquela operação como a derivada covariante (associada à conexão afim) do vetor

$$
\nabla_{\alpha} V^{\beta} \equiv \partial_{\alpha} V^{\beta}+\Gamma_{\alpha \gamma}^{\beta} V^{\gamma}=J_{\alpha}^{\mu} J_{\nu}^{\beta}\left(\partial_{\mu} V^{\nu}+\Gamma_{\mu \lambda}^{\nu} V^{\lambda}\right)
$$

Com argumentos similares aos anteriores, pode-se construir a derivada covariante de escalares, 1-formas e em geral de qualquer tensor,

$$
\begin{aligned}
& \text { escalar } \quad \nabla_{\mu} \phi=\partial_{\mu} \phi \\
& \text { 1-forma } \nabla_{\mu} \omega_{\nu}=\partial_{\mu} \omega_{\nu}-\Gamma_{\mu \nu}^{\lambda} \omega_{\lambda}, \\
& \text { tensor }(k, l) \quad \nabla_{\sigma} T_{\nu_{1} \ldots \nu_{l}}^{\mu_{1} \ldots \mu_{k}}=\partial_{\sigma} T_{\nu_{1} \ldots \nu_{l}}^{\mu_{1} \ldots \mu_{k}}+\Gamma_{\sigma \lambda}^{\mu_{1}} T_{\nu_{1} \ldots \nu_{l}}^{\lambda \mu_{2} \ldots \mu_{k}}+\cdots \\
& -\Gamma_{\sigma \nu_{1}}^{\lambda} T_{\lambda \nu_{2} \ldots \nu_{l}}^{\mu_{1} \ldots \mu_{k}}-\ldots
\end{aligned}
$$

ou seja, para um tensor $(k, l)$ por cada índice acima aparece um símbolo $+\Gamma_{\sigma \lambda}^{\mu_{n}}$ e por cada índice baixo aparece um símbolo $-\Gamma_{\sigma \nu_{m}}^{\lambda}$.

Como se mencionou ao final de 2.2.3, com um enfoque axiomático na definição da derivada covariante (por exemplo [24]) os símbolos que aparecem na derivada em geral não são os de Christoffel (2.37). Porém, a conexão afim podem ser obtida impondo simetria e que a derivada covariante do tensor métrico $g_{\mu \nu}$ seja nula, caso no qual se diz que a derivada covariante é compatível com o tensor métrico.

Dentre as possibilidades que abre o conceito de derivada covariante está a definição de transporte paralelo sobre a variedade. Em um espaço plano o transporte de um vetor de um ponto para outro pode ser feito mantendo-o paralelo, isto pode ser feito em espaços mais gerais. A derivada covariante (geralmente denotada $\nabla_{\sigma}$ ) quantifica a mudança imediata de um campo tensorial em comparação com o que seria se ele for transportado paralelamente. Em um espaço curvo o transporte paralelo vai depender da escolha do caminho. Se define [24] a equação de transporte paralelo do tensor $\boldsymbol{T}$ de tipo $(k, l)$ ao longo do caminho $x^{\mu}(\lambda)$ por meio da solução da equação

$$
\left(\frac{D}{d \lambda} \boldsymbol{T}\right)_{\nu_{1} \ldots \nu_{l}}^{\mu_{1} \ldots \mu_{k}} \equiv \frac{d x^{\sigma}}{d \lambda} \nabla_{\sigma} T_{\nu_{1} \ldots \nu_{l}}^{\mu_{1} \ldots \mu_{k}}=0
$$

ou seja, anulando a derivada direcional covariante $D / d \lambda$ do tensor de forma que as suas com- 
ponentes não mudem ao longo do caminho. Assim, o transporte paralelo de um vetor vai ser

$$
\frac{d V^{\mu}}{d \lambda}+\frac{d x^{\sigma}}{d \lambda} \Gamma_{\sigma \rho}^{\mu} V^{\rho}=0
$$

Se a conexão for compatível com o tensor métrico, este será transportado paralelamente ao longo de qualquer caminho. Por outro lado, o produto interno de vetores transportados paralelamente é conservado, ou seja, se preserva a norma.

Associado com o transporte paralelo está o conceito de geodésica. A geodésica é a generalização no espaço curvo de uma linha reta no espaço euclidiano. Também pode-se pensar como aquele caminho que transporta paralelamente os seus vetores tangentes. Aqueles serão os mesmos conceitos se a conexão for a afim. O transporte paralelo de vetores tangentes é dado por

$$
\frac{d^{2} x^{\mu}}{d \lambda^{2}}+\Gamma_{\sigma \rho}^{\mu} \frac{d x^{\sigma}}{d \lambda} \frac{d x^{\rho}}{d \lambda}=0
$$

e a equação (2.48) é a equação geodésica geral com parâmetro afim arbitrário. Mudando em (2.35) o tempo próprio por $\sigma=a \tau+b$ pode-se notar que a equação da geodésica fica invariante. Qualquer $\sigma$ relacionado com o tempo próprio ou com o parâmetro $\lambda$ de (2.48) assim, é conhecido como parâmetro afim. Com um parâmetro mais geral $\alpha(\lambda)$ vai-se ter

$$
\frac{d^{2} x^{\mu}}{d \alpha^{2}}+\Gamma_{\sigma \rho}^{\mu} \frac{d x^{\sigma}}{d \alpha} \frac{d x^{\rho}}{d \alpha}=f(\alpha) \frac{d x^{\mu}}{d \alpha}
$$

onde

$$
f(\alpha)=-\left(\frac{d^{2} \alpha}{d \lambda^{2}}\right)\left(\frac{d \alpha}{d \lambda}\right)^{-2}
$$

Se partirmos de uma equação como (2.49), sempre podemos conseguir um parâmetro afim $\lambda(\alpha)$ para o qual a equação da geodésica na forma (2.48) é satisfeita.

O transporte paralelo de um vetor ao longo de um caminho fechado pode medir a curvatura do conjunto limitado pelo caminho ao observar a mudança em dito vetor depois de completar o ciclo ao voltar ao ponto $p$. Essa mudança pode ser considerada como uma transformação do vetor no ponto $p$ e deve estar caracterizada por um tensor antissimétrico nos índices que caracterizam o caminho, já que um intercâmbio de eles sugere uma viagem na direção oposta [24]. Em um espaço sem torção o tensor de curvatura de Riemann é dado por

$$
R_{\sigma \mu \nu}^{\rho}=\partial_{\mu} \Gamma_{\nu \sigma}^{\rho}-\partial_{\nu} \Gamma_{\mu \sigma}^{\rho}+\Gamma_{\mu \lambda}^{\rho} \Gamma_{\nu \sigma}^{\lambda}-\Gamma_{\nu \lambda}^{\rho} \Gamma_{\mu \sigma}^{\lambda}
$$


Se o tensor de Riemann é nulo em algum ponto sempre podemos construir um sistema de coordenadas no qual as componentes do tensor métrico sejam constantes. As vezes vai ser conveniente trabalhar com um tensor que tenha todos os seus índices abaixo

$$
R_{\rho \sigma \mu \nu}=g_{\rho \lambda} R_{\sigma \mu \nu}^{\lambda}
$$

Associado com o tensor de curvatura existe uma identidade importante, a identidade de Bianchi

$$
\nabla_{[\lambda} R_{\rho \sigma] \mu \nu}=0
$$

Pode-se também tomar contrações no tensor de Riemann (2.51) mas apenas existe uma independente [24], o tensor de Ricci, o qual é simétrico

$$
R_{\mu \nu}=R_{\mu \lambda \nu}^{\lambda}
$$

Uma outra quantidade que vai ser importante é o traço do tensor de Ricci conhecido como escalar de Ricci

$$
R=R_{\mu}^{\mu}=g^{\mu \nu} R_{\mu \nu} .
$$

Tanto (2.54) como (2.55) têm toda a informação sobre a parte com traço do tensor de curvatura de Riemann [24]. Em termos de (2.54) e (2.55), a identidade (2.53) pode ser escrita como

$$
\nabla^{\mu} R_{\rho \mu}=\frac{1}{2} \nabla_{\rho} R
$$

\subsection{Equações de campo}

O princípio de covariância geral 2.2.2 sugere que as equações da física tem que ser escritas na linguagem tensorial. Na busca de uma generalização da equação de Poisson $\nabla^{2} \Phi=4 \pi \rho$, em que $\Phi$ é o potencial gravitacional e $\rho$ é a densidade de energia associada à massa que gera o campo, vai ser importante conseguir um tensor que leve em conta a densidade de energia associada à massa em uma das suas componentes, e além disso o caráter relativístico da equivalência nas formas de energia e a massa. Na generalização da equação de Poisson o tensor de energia momento $T_{\mu \nu}$ se considera como a fonte de campo gravitacional e de aí a sua importância em relatividade geral. Existem várias definições para o tensor de energia momento [21], mas todas 
concordam com o fato que a conservação da energia obtém-se com a equação

$$
\nabla_{\mu} T^{\mu \nu}=0 .
$$

O tensor de energia momento depende do sistema em particular sob estudo. No capítulo 4 se tratam alguns sistemas de interesse neste trabalho.

As equações de Einstein não são deduzíveis de um princípio básico. Elas são postuladas e aceitas válidas. Indutivamente, levando em conta os princípios de equivalência e de covariância geral e partindo da equação de Poisson, pode-se conseguir a forma que o conjunto de equações que descrevem o campo gravitacional deve ter a menos de uns parâmetros livres. Considerando a concordância que tem que existir com o limite clássico (velocidades baixas, campos gravitacionais fracos, campos estacionários) e a conservação da energia se consegue fixar os parâmetros em termos de constantes físicas e numéricas.

Baseado no sucesso que tem tido a mecânica clássica, e o princípio de mínima ação, um enfoque variacional postulando uma ação é uma forma de conseguir as equações. Nesta forma também se considera o princípio de equivalência e de covariância geral. A métrica será o objeto que se deseja conhecer para um sistema de fontes especificado pelo tensor de energia momento e daí pode-se conseguir informação sobre as trajetórias de corpos no espaço-tempo. Quanto ao enfoque variacional, a ação tem duas contribuições, a primeira é chamada ação de Hilbert, ou de Einstein-Hilbert [24, 25, 21]

$$
S_{H}\left[g_{\alpha \beta}\right]=\int \sqrt{-g} R d^{4} x,
$$

em que, $R$ é o escalar de Ricci (2.55), e $g$ é o determinante da métrica, a raiz faz que o elemento de volume seja invariante sob transformações gerais de coordenadas. A variação da ação de Einstein-Hilbert com respeito a métrica conduz as equações de campo de Einstein no vácuo

$$
\frac{1}{\sqrt{-g}} \frac{\delta S_{H}}{\delta g^{\mu \nu}}=R_{\mu \nu}-\frac{1}{2} R g_{\mu \nu}=0
$$

O tensor $R_{\mu \nu}$ envolve derivadas segundas da métrica. São dez equações não lineares e independentes para achar as dez componentes do tensor métrico.

A segunda contribuição para a ação tem a ver com o tensor de energia momento. No caso de uma descrição da matéria por meio de uma teoria de campos, existe uma Lagrangiana $L_{M}=$ 
$L_{M}\left(\phi, \partial_{\alpha} \phi, \ldots, g_{\alpha \beta}, \ldots\right)$ que acopla a matéria com o campo gravitacional [21]. A variação da ação correspondente respeito da métrica vai ser proporcional ao tensor de energia momento para o campo $\phi$. O mesmo pode ser mostrada para outros campos [21]. Logo, se assume que uma boa definição do tensor de energia momento é dada por

$$
T_{\alpha \beta}=-\frac{2}{\sqrt{-g}} \frac{\delta}{\delta g^{\alpha \beta}} S_{M}\left[\phi, g_{\alpha \beta}\right]
$$

Assim, a ação que considera o campo gravitacional e o seu acoplamento com a matéria se escreve

$$
S=\frac{1}{16 \pi} S_{H}+S_{M}
$$

e a variação da ação com respeito a métrica implica as equações de Einstein, com fontes dadas pelo tensor de energia momento $T_{\alpha \beta}[21,25,24]$

$$
\frac{1}{\sqrt{-g}} \frac{\delta S}{\delta g^{\mu \nu}}=0 \Rightarrow R_{\mu \nu}-\frac{1}{2} R g_{\mu \nu}=8 \pi T_{\mu \nu}
$$

que são as equações de Einstein na sua forma usual. Mais um termo pode ser incluso na ação e pode-se interpretar como associado à densidade de energia do vácuo e inclui a famosa constante cosmológica $\Lambda$. A ação total para descrever a dinâmica do campo com fontes e constante cosmológica, se escreve conforme

$$
S=\frac{1}{16 \pi} \int \sqrt{-g}(R-2 \Lambda) d^{4} x+S_{M},
$$

as equações de Einstein com a constante cosmológica são

$$
R_{\mu \nu}-\frac{1}{2} R g_{\mu \nu}+\Lambda g_{\mu \nu}=8 \pi T_{\mu \nu}
$$

Por causa da simetria dos tensores envolvidos em ambos lados são dez equações para achar dez componentes do tensor métrico. Mas existem as quatro relações de Bianchi (2.56) que reduzem a seis as equações independentes. O número de incógnitas se reduz a seis como esperado por causa da liberdade no tensor métrico na escolha das quatro coordenadas espaço-temporais.

O tensor $g_{\mu \nu}$ solução das equações de Einstein (2.64) define a geometria do espaço-tempo na relatividade geral com constante cosmológica $\Lambda$ e fonte de campo $T_{\mu \nu}$. As simetrias que possam vir a existir na geometria do espaço-tempo estão relacionadas com o tensor métrico. Portanto, a 
busca de simetrias na geometria do espaço-tempo é uma ferramenta valiosa na busca de soluções das equações de Einstein. No próximo capítulo serão apresentadas soluções das equações de Einstein com simetrias especias.

A caracterização das simetrias que possui um espaço-tempo dado podem ser expressas em termos da existência de campos vetoriais ao longo dos quais a derivada de Lie do tensor métrico para esse espaço-tempo é nula. Usando a equação (B.12) e o fato de ser a conexão afim compatível com o tensor métrico, temos que

$$
\mathcal{L}_{K} g_{\mu \nu}=\nabla_{\mu} K_{\nu}+\nabla_{\nu} K_{\mu}=0
$$

é a condição para que exista uma isometria. O campo vetorial $K$ é chamado campo vetorial de Killing e a equação (2.65) equação de Killing para o campo $\boldsymbol{K}$. Caso seja considerado um sistema de coordenadas no qual o parâmetro que define a curva integral do campo $\boldsymbol{K}$ for uma das coordenadas, por exemplo $x^{\sigma_{*}}=\lambda$ ( $\sigma_{*}$ é um índice fixo) a equação (2.65) pode ser expressa de uma forma particularmente simples

$$
\mathcal{L}_{K} g_{\mu \nu}=\frac{\partial g_{\mu \nu}}{\partial x^{\sigma_{*}}}=0
$$

e o vetor $\boldsymbol{K}$ é dito ser o gerador da isometria [24]. Sempre é possível encontrar um sistema de coordenadas adaptado ao campo vetorial $\boldsymbol{K}$ em cujo caso se escreve $\boldsymbol{K}=\delta_{\sigma_{*}}^{\mu} \partial_{\mu}$. Da mesma forma, se existir algum sistema de coordenadas em que o tensor métrico for independente de alguma coordenada, então deve existir uma isometria e um campo vetorial de Killing associado. 


\section{Capítulo 3}

\section{Buracos negros e horizontes}

A primeira solução do conjunto de equações de Einstein (2.59) foi obtida em 1915 pouco tempo antes de ser publicadas as equações de Einstein na sua forma final covariante em 1916 [32]. Karl Scwarzschild achou uma solução no vácuo estática e esfericamente simétrica conhecida hoje como solução de Schwarzschild. O elemento de linha de Schwarzschild pode ser expresso como um caso particular de métricas da forma

$$
d s^{2}=-f(r) d t^{2}+f(r)^{-1} d r^{2}+r^{2} d \Omega^{2}
$$

em que $d \Omega^{2}=d \theta^{2}+\sin ^{2} \theta d \phi^{2}$ (invariante sob rotações) é o elemento de linha da esfera $S^{2}$. Existem várias escolhas na função $f(r)$ que modelam diferentes situações físicas. No caso da métrica de Schwarzschild

$$
f(r)=1-\frac{2 m}{r}
$$

onde a constante $m$ é chamada de massa geométrica e lembrando que foi escolhido o sistema de unidades naturais em que $c=G=1$. Se interpretarmos a solução de Schwarzschild como sendo a métrica do espaço-tempo no exterior de um objeto compacto na origem de coordenadas $[33,34]$ (daqui para frente coordenadas de Schwarzschild), se encontra que a massa geométrica é a massa do objeto no sentido Newtoniano. A métrica de Schwarzschild é assintoticamente plana, o que significa que para valores da coordenada radial $r$ grandes o suficiente em comparação a $m$, o elemento de linha pode ser aproximado pela métrica de Minkowski. Obtém-se também a métrica de Minkowski quando $m=0$.

Observa-se de (3.1) que $\partial_{\phi} g_{\mu \nu}=0, \operatorname{logo} \boldsymbol{K}_{\phi}=\partial_{\phi}$ é um campo de Killing tipo espaço, já 
que $\boldsymbol{K}_{\phi} \cdot \boldsymbol{K}_{\phi}=r^{2} \sin ^{2} \phi$. Existem duas rotações independentes que podem ser feitas sobre o elemento de linha (3.1) definindo dois novos ângulos que substituem o papel de $\phi$ e que definem mais dois campos de Killing linearmente independentes $\boldsymbol{K}_{\bar{\phi}}=\partial_{\bar{\phi}}$ e $\boldsymbol{K}_{\hat{\phi}}=\partial_{\hat{\phi}}$. Esses campos satisfazem a relação de comutação da álgebra de Lie de $S O(3)$ e têm órbitas fechadas [34]

$$
\left[\boldsymbol{K}_{i}, \boldsymbol{K}_{j}\right]=\epsilon_{i j}{ }^{k} \boldsymbol{K}_{k}
$$

com $\{i, j, k\}=\{\phi, \bar{\phi}, \hat{\phi}\}$ e $\epsilon_{i j k}$ é o símbolo de permutação antissimétrico no espaço plano tridimensional tal que para uma permutação par de $i, j, k$ temos $\epsilon_{i j k}=1$, para uma permutação impar de $i, j, k$ temos $\epsilon_{i j k}=-1$, e é nulo em qualquer outro caso. Um espaço-tempo dotado de tais campos vetoriais tipo espaço é dito ser esfericamente simétrico. É o caso do espaço-tempo de Schwarzschild [34].

De (3.1) pode-se notar que $\partial_{t} g_{\mu \nu}=0, \operatorname{logo} \boldsymbol{K}_{t}=\partial_{t}$ é um campo de Killing tipo tempo na região $r>2 m$, já que $\boldsymbol{K}_{t} \cdot \boldsymbol{K}_{t}=-(1-2 m / r)$. Um espaço-tempo dotado de um campo vetorial de Killing tipo-tempo é dito ser estacionário. O espaço-tempo de Schwarzschild (com $r>2 m$ ) é estacionário [34].

Por outro lado, seja uma família de hipersuperfícies no espaço-tempo definidas por meio de alguma função das coordenadas igualada a uma constante $f(\boldsymbol{x})=c$. Um campo vetorial $\boldsymbol{X}$ é dito ser ortogonal à hipersuperfície $f(\boldsymbol{x})=c$ se for proporcional ao vetor normal à hipersuperfície $\boldsymbol{n}$, em cada ponto da variedade [22, 34],

$$
\boldsymbol{X}=\lambda(\boldsymbol{x}) \boldsymbol{n}
$$

O gradiente da função, $\boldsymbol{d} f=\partial_{\mu} f d x^{\mu}$ é a 1-forma ortogonal à hipersuperfície. O vetor $\boldsymbol{n}$ é definido como o dual do gradiente $\boldsymbol{n}=\boldsymbol{g}^{-1}(\boldsymbol{d} f)=g^{\alpha \beta} \partial_{\beta} f \partial_{\alpha}$.

Pode ser mostrado [34] que a condição de campo vetorial ortogonal à hipersuperfície se reduz em satisfazer a seguinte igualdade

$$
X_{[\sigma} \nabla_{\mu} X_{\nu]}=0
$$

Um espaço-tempo é dito ser estático se admitir um campo vetorial de Killing tipo tempo ortogonal à hipersuperfície [34]. Pode ser mostrado que o campo de Killing $\boldsymbol{K}_{t}=\partial_{t}$ satisfaz (3.5), logo o espaço-tempo de Schwarzschild é estático na região $r>2 m$. 


\subsection{Espaço-tempo de Schwarzschild e extensão maximal}

A única solução das equações de Einstein (2.59) que descreve o espaço-tempo vazio fora de um objeto esfericamente simétrico de massa $m$, é estática e está dada por (3.1) com a $f(r)$ de (3.2). Essa afirmação é conhecida como teorema de Birkhoff [24, 21, 22, 34].

Porém, existem cartas de coordenadas nas quais são acessíveis outras regiões das quais não se têm conhecimento desde o olhar das coordenadas de Schwarzschild e é considerada uma extensão do espaço-tempo que as inclua. A carta de coordenadas isotrópicas por exemplo, resulta de uma transformação na coordenada $r$ e consegue expressar as fatias de $t$ constante como hipersuperfícies isotrópicas conformes à métrica de Minkowski. A transformação de coordenadas pode ser construída partindo da fatorização seguinte,

$$
d s^{2}=-\left(1-\frac{2 m}{r}\right) d t^{2}+\left(1-\frac{2 m}{r}\right)^{-1}\left[d r^{2}+\left(1-\frac{2 m}{r}\right) r^{2} d \Omega^{2}\right]
$$

onde se faz a seguinte identificação

$$
\begin{aligned}
\lambda^{2}(r) d \rho^{2} & =d r^{2} \\
\lambda^{2}(r) \rho^{2} & =\left(1-\frac{2 m}{r}\right) r^{2} .
\end{aligned}
$$

O sistema pode ser resolvido para $\lambda(r)$ quando a condição $r \rightarrow \infty$ se $\rho \rightarrow \infty$ for imposta [34]. Com a escolha adequada de constantes de integração, em particular $r_{0}=2 m$ e $\rho_{0}=m / 2$, a transformação entre cartas é dada pela expressão

$$
r=\rho\left(1+\frac{m}{2 \rho}\right)^{2}
$$

Porém, não é necessária a construção anterior. A troca de variáveis dada pela equação (3.9) pode ser considerada um ansatz. O elemento de linha de Schwarzschild em coordenadas isotrópicas é dado por

$$
d s^{2}=-\left(\frac{1-\frac{m}{2 \rho}}{1+\frac{m}{2 \rho}}\right)^{2} d t^{2}+\left(1+\frac{m}{2 \rho}\right)^{4}\left(d \rho^{2}+\rho^{2} d \Omega^{2}\right)
$$

Na métrica expressa nas coordenadas de Schwarzschild, o raio $r$ é conhecido como raio areal, o que significa que superfícies definidas por $t$ e $r$ constantes têm área $A=4 \pi r^{2}$. Nota-se de (3.10) que a coordenada $\rho$ não é o raio areal. 
Segue da definição da coordenada $\rho$ em (3.9) que por cada valor da coordenada $r$ no intervalo $(2 m, \infty)$ existem dois valores da coordenada ${ }^{1} \rho$, ou seja, a carta com as novas coordenadas cobre a região exterior a $r=2 m$ duas vezes com intervalos na coordenada $\rho$ diferentes definindo duas regiões idênticas mas independentes do espaço-tempo [21].

Em $r=2 m$, conhecido como raio de Schwarzschild, a função $f(r)$ (3.2) é nula e existe uma divergência em um dos coeficientes métricos nas coordenadas $(t, r, \theta, \phi)$. A solução de Schwarzschild ainda é solução das equações de Einstein no interior da hipersuperfície $r=2 m$ (quando o raio do objeto compacto de massa $m$ for menor que $2 m$ ) mas a função $f(r)$ torna-se negativa, logo a coordenada $t$ é tipo espaço e a coordenada $r$ tipo tempo. Sendo o elemento de linha dependente de $r$, o espaço-tempo no interior de $r=2 m$ passa a ser dinâmico.

As geodésicas radiais ( $\operatorname{com} \theta=\theta_{0}, \phi=\phi_{0}$ ) tipo luz que se aproximam à origem de coordenadas atingem o valor $r=2 m$ apenas quando $t$ tender a infinito, isto acontece para observadores estáticos nas coordenadas de Schwarzschild. Por outro lado, um observador em queda livre consegue atingir a superfície $r=2 m$ em um tempo próprio finito [21]. O tensor de curvatura ainda está definido nesse valor da coordenada radial, as equações de Einstein estão definidas ali e não se considera que exista uma singularidade na variedade [24].

Uma transformação de coordenadas pode eliminar a divergência que tem o coeficiente métrico $1 / f(r)$ em $r=2 m$. O matemático Frances Paul Painlevé em 1921 e independentemente o oftalmologista Sueco Allvar Gullstrand em 1922, encontraram um sistema de coordenadas no qual o espaço-tempo de Schwarzschild não tinha a conhecida divergência em $r=2 m$ [32]. As coordenadas de Painlevé-Gullstrand resultam de uma mudança na coordenada temporal na métrica de Schwarzschild. As novas coordenadas estão adaptadas a observadores geodésicos aproximando-se ou afastando-se radialmente da inomogeneidade de massa $m$ com tempo próprio $\tau=T(t, r)$ [21]. A transformação de coordenadas, na região $r>2 m$ é dada por

$$
T_{ \pm}(t, r)=t \pm 2(2 m r)^{1 / 2} \pm 2 m \ln \left|\frac{(r / 2 m)^{1 / 2}-1}{(r / 2 m)^{1 / 2}+1}\right|
$$

onde o sinal escolhido depende do sinal de $\dot{r}$, sendo positivo quando $\dot{r}<0$ e sendo negativo

\footnotetext{
${ }^{1}$ Pode ser notado quando se escreve a coordenada $\rho$ em termos de $r$.
} 
quando $\dot{r}>0$. O elemento de linha no novo sistema de coordenadas é

$$
d s^{2}=-d T^{2}+\left(d r \pm \sqrt{\frac{2 m}{r}} d t\right)^{2}+r^{2} d \Omega^{2}
$$

o qual é regular em $r=2 m$. A métrica obtida (3.12) é a mesma no caso de fazer a mudança de variáveis na região $r<2 m$ apesar de ter uma coordenada $T(t, r)$ diferente. Por outro lado, o espaço-tempo de Schwarzschild possui uma singularidade em $r=0$ onde escalares derivados do tensor de curvatura não estão definidos [24]. Neste caso a divergência não pode ser removida com uma troca de sistema de coordenadas.

Uma outra forma de rotular o espaço-tempo é usando coordenadas adaptadas a feixes de luz geodésicos. Da métrica de Schwarzschild (3.1), a condição $d s^{2}=0 \operatorname{com} \theta$ e $\phi$ fixos, implica que geodésicas nulas radiais satisfazem a equação diferencial

$$
\frac{d r}{d t}= \pm\left(1-\frac{2 m}{r}\right)
$$

cuja solução é

$$
t_{l}(r)= \pm r \pm 2 m \ln \left|\frac{r}{2 m}-1\right|+t_{l}\left(r_{0}\right) \mp r_{0} \mp 2 m \ln \left|\frac{r_{0}}{2 m}-1\right|
$$

onde o sub-índice $l$ quer dizer "luz". Se observa que os últimos três termos em (3.14) dependem apenas de condições iniciais mas cada escolha corresponde com uma geodésica nula diferente. Por simplicidade se define

$$
r^{*}=r+2 m \ln \left|\frac{r}{2 m}-1\right|,
$$

conhecida como coordenada de Regge-Wheeler ou coordenada tartaruga [21]. É possível definir novas coordenadas para as quais geodésicas nulas se correspondem com valores constantes delas. A partir de (3.14), se tem

$$
\begin{aligned}
t_{l}^{(\text {out })}-r^{*}(r) & =t_{l}\left(r_{0}\right)-r_{0}-2 m \ln \left|\frac{r_{0}}{2 m}-1\right| \equiv u\left(t_{l}\left(r_{0}\right), r_{0}\right), \\
t_{l}^{(i n)}+r^{*}(r) & =t_{l}\left(r_{0}\right)+r_{0}+2 m \ln \left|\frac{r_{0}}{2 m}-1\right| \equiv v\left(t_{l}\left(r_{0}\right), r_{0}\right) .
\end{aligned}
$$

Ou seja, se definem as coordenadas de tempo retardado $u$ (adaptada a geodésicas nulas out- 
going) ou avançado $v$ (adaptada a geodésicas nulas ingoing) como

$$
\begin{aligned}
& u(t, r)=t-r^{*}(r), \\
& v(t, r)=t+r^{*}(r),
\end{aligned}
$$

também conhecidas como coordenadas nulas. Com a mudança de coordenadas (3.18) ou (3.19) no elemento de linha de Schwarzschild se obtém a versão retardada ou avançada da métrica de Eddington-Finkelstein

$$
\begin{aligned}
d s^{2} & =-\left(1-\frac{2 m}{r}\right) d u^{2}-2 d u d r+r^{2} d \Omega^{2} \\
d s^{2} & =-\left(1-\frac{2 m}{r}\right) d v^{2}+2 d v d r+r^{2} d \Omega^{2}
\end{aligned}
$$

Da mesma forma que na métrica de Painlevé-Gullstrand (3.12), as métricas (3.20) e (3.21) não têm a divergência no raio de Schwarzschild e a região interna a $r=2 m$ é agora explicitamente acessível (desde que o objeto de massa $m$ tenha um raio $R_{0}<2 m$ ). Porém, uma análise dos cones de luz de (3.21) mostra que para valores da coordenada radial $r<2 m$ os caminhos geodésicos dirigidos ao futuro avançam apenas na direção em que o $r$ diminui. Nem existe a possibilidade de ficar parado em valores fixos de $r$. De fato, a superfície $r=2 m$ é nula, e desta forma ficar na superfície não é possível para nenhuma partícula massiva.

Uma vez na região interior à superfície $r=2 m$ nada pode sair pela própria estrutura causal do espaço-tempo. A coordenada radial se torna tipo tempo e o avanço à singularidade em $r=0$ é inevitável. A superfície $r=2 m$ é chamada horizonte de eventos e separa a região interna do infinito. A região interna é chamada de buraco negro. Porém, uma análise detalhada das métricas (3.20) e (3.21) mostra que elas definem regiões diferentes do espaço-tempo [21, 24].

É possível usar simultaneamente as coordenadas nulas (3.18) e (3.19) com o qual a métrica de Schwarzschild pode ser expressa na forma

$$
d s^{2}=-\left(1-\frac{2 m}{r(u, v)}\right) d u d v+r^{2}(u, v) d \Omega^{2}
$$

onde $r=r(u, v)$ está definido implicitamente pela equação

$$
r^{*}=r+2 m \ln \left|\frac{r}{2 m}-1\right|=\frac{1}{2}(u-v) .
$$


Apesar de não ter divergências na métrica expressa nas coordenadas $(u, v, \theta, \phi)$, aparece de novo um problema em $r=2 m$ [33]. Por exemplo o determinante da métrica nessas coordenadas é nulo no raio de Schwarzschild. Se considera a seguinte transformação de coordenadas que elimina o problema

$$
\begin{aligned}
& U=-4 m e^{-u / 4 m}, \\
& V=4 m e^{v / 4 m} .
\end{aligned}
$$

Em termos das coordenadas $U$ e $V$, o elemento de linha (3.22) é escrito

$$
d s^{2}=-\frac{2 m}{r(U, V)} e^{-r(U, V) / 2 m} d U d V+r^{2}(U, V) d \Omega^{2}
$$

em que $r=r(U, V)$ é agora definido implicitamente pelo produto $U V$

$$
16 m^{2}\left(\frac{r}{2 m}-1\right) e^{r / 2 m}=-U V .
$$

A métrica (3.26) é continua e regular em $r=2 m$. O domínio das coordenadas $U, V$ é o intervalo $(-\infty, \infty)$. Mais uma troca pode ser feita com o propósito de descrever o espaço-tempo todo numa região limitada. São as coordenadas nulas retardada e avançada re-escaladas [33]

$$
\begin{aligned}
& \tilde{u}=2 \tan ^{-1}\left(\frac{U}{4 m}\right), \\
& \tilde{v}=2 \tan ^{-1}\left(\frac{V}{4 m}\right) .
\end{aligned}
$$

As novas variáveis descrevem a extensão maximal do espaço-tempo de Schwarzschild em termos de 4 regiões diferentes. O domínio das coordenadas $\tilde{u}, \tilde{v}$ satisfaz as desigualdades $-\pi<$ $\tilde{u}<\pi,-\pi<\tilde{v}<\pi \mathrm{e}-\pi<\tilde{u}+\tilde{v}<\pi$. Os diagramas de Carter-Penrose, também chamados de diagramas de Penrose, são ferramentas úteis na descrição do espaço-tempo [21, 24, 22].

Na figura 3.1 se observa o diagrama de Penrose da extensão maximal do espaço-tempo de Schwarzschild. Cada ponto no diagrama representa uma esfera de raio $r$. Cada linha é uma hipersuperfície de $r$ constante. Qualquer uma das regiões quadradas laterais se correspondem com o espaço-tempo descrito pela métrica de Schwarzschild, essas regiões estão delimitadas pelos horizontes passado e futuro em $r=2 m$, por um lado e do outro lado pelos infinitos nulos passado e futuro, denotados por $\mathcal{I}^{-}$e $\mathcal{I}^{+}$respectivamente, os quais se intersetam no 


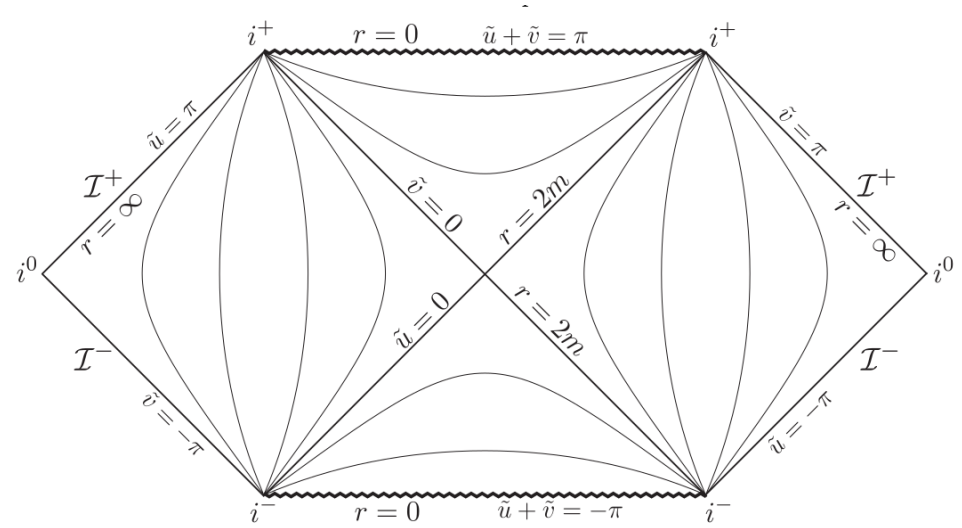

Figura 3.1: Diagrama de Penrose da extensão maximal do espaço-tempo de Schwarzschild. Com a transformação de coordenadas (3.28) e (3.29) os infinitos podem ser representados nesse diagrama. Todas as linhas no diagrama representam hipersuperfícies de $r$ constante. Figura obtida na referência [33].

infinito espacial $i^{0}$. O infinito futuro tipo tempo $i^{+}$pode ser alcançado quando $t \rightarrow \infty$ com $r$ fixo. O infinito passado tipo tempo $i^{-}$pode ser alcançado quando $t \rightarrow-\infty$ com $r$ fixo. As regiões triangulares superior e inferior representam um buraco negro e um buraco branco ${ }^{2}$ respectivamente cuja descrição pode ser obtida com as coordenadas de Eddington-Finkelstein (3.20) e (3.21).

O horizonte de eventos corresponde a $U=0(\tilde{u}=0)$ e/ou $V=0(\tilde{v}=0)$. E a singularidade à hipérbole de dois ramos $U V=1(\tilde{u}+\tilde{v}= \pm \pi)$ A singularidade em $r=0$ é tipo espaço pelo fato de ser $r$ uma coordenada tipo tempo quando $r<2 m$, da mesma forma em que hipersuperfícies de $t$ constante quando $r>2 m$ são tipo espaço.

Existem várias versões do diagrama de Penrose para a extensão maximal do espaço-tempo de Schwarzschild obtidas por meio de mudanças adicionais nas coordenadas [21, 24, 22]. Com a transformação para as coordenadas tipo espaço e tipo tempo $R, T$ [33] dada por $U=T+$ $R, V=T-R$ para $r>2 m$, e $U=R-T, V=R+T$ para $r<2 m$ o diagrama na figura 3.1 preserva a sua forma, mas as geodésicas tipo luz são representadas por meio de linhas retas a $45^{\circ}$, logo, qualquer raio de luz ingoing acaba sempre na singularidade do buraco negro. No caso do buraco branco qualquer feixe de luz sempre sai do buraco e termina em $\mathcal{I}^{+}$.

Seja $S$ um conjunto de eventos na variedade espaço-temporal $\mathcal{M}$, o conjunto $J^{+}(S)$ se define como o conjunto de eventos $q$ que são atingidos por curvas tipo tempo ou nulas apontando para o futuro que partiram de pontos $p \in S$ e é chamado futuro causal de $S$. O conjunto $J^{-}(S)$ se define como o conjunto de eventos $q$ dos quais partem curvas tipo tempo ou nulas apontando

\footnotetext{
${ }^{2}$ Entendido como sendo a reversão temporal do buraco negro, ver [21, 24] por exemplo.
} 
para o futuro que atingem pontos $p \in S$ e é chamado passado causal de $S$ [22, 24]. Logo, a definição matemática padrão de buraco negro é o conjunto $\mathcal{B}$ da variedade espaço-temporal $\mathcal{M}$ formado por todos os eventos $p$ que não pertencem ao passado causal do infinito nulo futuro [22], ou seja

$$
\mathcal{B}=\mathcal{M}-J^{-}\left(\mathcal{I}^{+}\right)
$$

O horizonte de eventos $\mathcal{H}$ é definido como sendo a fronteira da região do buraco negro, ou de forma equivalente, a fronteira do passado causal do infinito nulo futuro

$$
\mathcal{H}=\partial \mathcal{B}=\partial\left(J^{-}\left(\mathcal{I}^{+}\right)\right)
$$

As definições apresentadas descrevem o buraco negro como sendo uma propriedade global da estrutura causal de um espaço-tempo inteiro e não em termos de quantidades definidas apenas localmente. A localização do horizonte de eventos não é possível do ponto de vista real. Um observador precisaria esperar tempo infinito afastado do buraco negro uma distância infinita para ter certeza de qual a região da qual não é recebida sinal nenhuma. Não é possível identificar propriamente a região de buraco negro sem esse conhecimento global [23].

Levando em conta que as definições (3.30) e (3.31) descrevem uma situação ideal é razoável argumentar que em situações físicas reais aproximadamente estáticas (o que depende do contexto) pode ser substituído o $\mathcal{I}^{+}$com um conjunto de observadores muito afastados do buraco negro que esperam por tempos longes mas finitos [23]. Em situações dinâmicas já não será mais possível aquela substituição.

Dada essa dificuldade, várias outras caracterizações de buraco negro foram criadas, algumas delas quase locais. O que significa que a definição é construída em termos de quantidades não necessariamente definidas na variedade toda. Apresentaremos algumas delas para serem qualitativamente comparadas e poder justificar logo depois a nossa escolha no presente trabalho.

\subsection{Algumas caracterizações para horizontes}

Muitos autores que abordam a temática [24, 22, 35, 36, 37] por exemplo, incluem a definição de horizonte de Killing como parte importante na teoria desenvolvida até hoje sobre buracos negros. Seja $\left(\mathcal{M}, g_{\mu \nu}\right)$ um espaço-tempo. Uma 3-superfície $\mathcal{H}$ é dita ser um horizonte de 
Killing se for tangente em todo lugar a algum campo vetorial de Killing $\boldsymbol{\xi}$ definido sobre todo $\mathcal{M}$ o qual é nulo sobre o $\mathcal{H}[23]$.

Normalmente a normalização do campo vetorial é tal que $\boldsymbol{\xi} \cdot \boldsymbol{\xi}=-1$ no infinito. A definição precisa de um campo vetorial de Killing definido em todo o espaço-tempo. Existe uma definição alternativa que relaxa a condição da existência do campo vetorial restringindo-lo a uma vizinhança de $\mathcal{H}$ ficando aberta a possibilidade de descrever horizontes que talvez estão temporalmente em equilíbrio com o seu entorno. Em espaço-tempos estacionários e assintoticamente planos os horizontes de Killing coincidem com os horizontes de eventos [21, 22, 23]. Porém, existe o problema de que nem sempre os horizontes de Killing estão associados com espaço-tempos de buraco negro [21,23].

Outra caracterização é feita em termos das chamadas trapped surfaces, as quais formam a base de uma ampla gama de caracterizações mais recentes [23]. As trapped surfaces estão definidas em termos de expansões de campos vetoriais tangentes a uma família de linhas geodésicas nulas (congruência geodésica nula, [21, 22]) ortogonais a hipersuperfícies. As expansões são os divergentes dos campos nulos considerados quando a parametrização for afim [21].

Sejam dois campos vetoriais nulos linearmente independentes $n, \ell$ ortogonais à superfície fechada $S$ apontando para dentro e para fora respectivamente e dirigidos ao futuro, com a escolha $\boldsymbol{n} \cdot \boldsymbol{\ell}=-1$. A superfície $S$ é dita ser uma trapped surface se as expansões dos campos $\boldsymbol{n}$ e $\ell$ são ambas negativas [23]. Ou seja, se

$$
\begin{gathered}
\theta_{(n)}=h^{\alpha \beta} \nabla_{\alpha} n_{\beta}<0, \\
\theta_{(\ell)}=h^{\alpha \beta} \nabla_{\alpha} \ell_{\beta}<0,
\end{gathered}
$$

onde $h_{\alpha \beta}$ é a métrica transversa aos campos vetoriais $\boldsymbol{n}$ e $\ell$ e é dada por

$$
h_{\alpha \beta}=g_{\alpha \beta}+\ell_{\alpha} n_{\beta}+\ell_{\beta} n_{\alpha} .
$$

A métrica transversa $h_{\alpha \beta}$ tem a propriedade de ser puramente espacial e de agir como um tensor de projeção sobre a 2-superfície fechada $S$, de forma que $h_{\alpha \beta} \ell^{\alpha}=h_{\alpha \beta} n^{\alpha}=0$. Também se tem $h_{\beta}^{\alpha} h_{\gamma}^{\beta}=h_{\gamma}^{\alpha}$.

A condição de normalização $(\ell \cdot \boldsymbol{n}=-1)$ não é necessária e as vezes interfere com outras exigências que podemos pedir aos campos $\boldsymbol{\ell}$ e $\boldsymbol{n}$ [37]. Nesse caso a expressão para a métrica 
transversa pode ser modificada na forma seguinte

$$
h_{\alpha \beta}=g_{\alpha \beta}-\frac{\ell_{\alpha} n_{\beta}+\ell_{\beta} n_{\alpha}}{\ell^{\gamma} n_{\gamma}} .
$$

Considerando o fato que os campos $\boldsymbol{n}$ e $\ell$ são ortogonais à superfície $S$, a métrica transversa $h_{\alpha \beta}$ é a métrica induzida sobre $S$ e as expansões (3.32) e (3.33) podem ser expressas em termos do determinante da métrica induzida ou alternativamente em termos da mudança na área da superfície $S$ ao longo das curvas integrais dos campos vetoriais nulos. Vemos que devido à simetria da métrica induzida $h_{\alpha \beta}$, a expansão pode ser escrita como [21]

$$
\theta_{(V)}=h^{\alpha \beta} \nabla_{\alpha} V_{\beta}=\frac{1}{2} h^{\alpha \beta}\left(\nabla_{\alpha} V_{\beta}+\nabla_{\beta} V_{\alpha}\right)=\frac{1}{2} h^{\alpha \beta} \mathcal{L}_{V} g_{\alpha \beta},
$$

onde $\boldsymbol{V}$ é $\boldsymbol{n}$ ou $\boldsymbol{\ell}$. Substituindo a métrica $g_{\alpha \beta}$ em termos da métrica induzida (3.34) e com o uso da seguinte relação para o determinante da métrica [21], válida também para a métrica induzida,

$$
\delta \sqrt{|g|}=\frac{1}{2} \sqrt{|g|} g^{\alpha \beta} \delta g_{\alpha \beta},
$$

temos que a expansão (3.36) vai se tornar

$$
\theta_{(V)}=\frac{1}{2} h^{\alpha \beta} \mathcal{L}_{\boldsymbol{V}} g_{\alpha \beta}=\frac{1}{\sqrt{h}} \mathcal{L}_{\boldsymbol{V}} \sqrt{h}
$$

Existe ainda uma relação entre o elemento de área de $S$ e a raiz do determinante da métrica induzida, $\delta A=\sqrt{h} d^{2} x$. Obtém-se que

$$
\begin{aligned}
& \theta_{(n)}=\frac{1}{\sqrt{h}} \mathcal{L}_{n} \sqrt{h}=\frac{1}{\delta A} \frac{d}{d \lambda_{n}} \delta A, \\
& \theta_{(\ell)}=\frac{1}{\sqrt{h}} \mathcal{L}_{\ell} \sqrt{h}=\frac{1}{\delta A} \frac{d}{d \lambda_{\ell}} \delta A,
\end{aligned}
$$

onde $\lambda_{n}$ e $\lambda_{\ell}$ são os parâmetros das curvas integrais de $\boldsymbol{n}$ e $\ell$. Existem teoremas gerais que garantem que os fortes campos gravitacionais de buracos negros estão caracterizados por trapped surfaces [23]. Sob determinadas condições de energia, espaço-tempos com um $\mathcal{I}^{+}$definido, qualquer weakly trapped surface (onde as expansões são apenas não positivas) deve conter no interior um buraco negro [23]. Temos também que, sem ter o $\mathcal{I}^{+}$definido, quando se satisfaz a condição de energia fraca qualquer trapped surface pode ser estendida em uma 3-superfície 
tipo espaço de área constante que sela causalmente seu interior do resto do espaço-tempo [23]. Encontrar uma trapped surface é condição suficiente para que exista uma singularidade no espaço-tempo em algum lugar no futuro causal [23].

Associado com a região do espaço-tempo na qual existem trapped surfaces está a sua fronteira. Se um espaço-tempo pode ser foliado por superfícies tridimensionais assintoticamente planas tipo espaço $\Sigma_{t}$, um ponto $q \in \Sigma_{t}$ é dito estar atrapado se ele fica em uma trapped surface em $\Sigma_{t}$ [23]. O horizonte aparente em $\Sigma_{t}$ é a fronteira da união de todos os pontos atrapados e sob determinadas condições de suavidade, pode ser mostrado que vai ser uma superfície na qual $\theta_{(\ell)}=0[23]$

De acordo com a definição anterior se entende que nem sempre é possível identificar a superfície onde $\theta_{(\ell)}=0$ com o horizonte aparente. Mas do ponto de vista prático [23], a definição é deixada do lado e o termo é usado para superfícies com $\theta_{(\ell)}=0$.

A definição de horizonte aparente é menos restritiva para Faraoni [37], que não considera que o espaço-tempo deve ser assintoticamente plano. Além disso a condição de anulação da expansão de campos nulos tem maior importância. Antes de apresentar a sua definição mostramos alguns conceitos relevantes relativos a 2-superfícies fechadas (alguns autores exigem que sejam superfícies tipo espaço, por exemplo [22, 35, 38]) em relação a campos geodésicos nulos ingoing e outgoing (com a convenção que usamos $\ell$ e $\boldsymbol{n}$ respectivamente). Uma superfície normal se corresponde $\operatorname{com} \theta_{(\ell)}>0$ e $\theta_{(n)}<0$, por exemplo na ausência de gravitação. Uma trapped surface para Faraoni [37] corresponde a $\theta_{(\ell)}<0$ e $\theta_{(n)}<0$ (sem impor a condição de normalização $\ell^{\alpha} n_{\alpha}=-1$ ). Uma marginally outer trapped surface (MOTS) (ou marginal) corresponde a $\theta_{(\ell)}=0$, e $\theta_{(n)}<0$. Uma untrapped surface é uma na qual $\theta_{(\ell)} \theta_{(n)}<0$. Uma anti-trapping surface corresponde a $\theta_{(\ell)}>0$ e $\theta_{(n)}>0$. Um marginally outer trapped tube (MOTT) é uma 3-superfície que pode ser totalmente foliada por MOTS.

Para Faraoni [37], um horizonte aparente futuro é o fecho de uma superfície (usualmente uma 3-superfície) a qual pode ser foliada por superfícies marginais. É uma superfície definida pelas condições seguintes sobre as fatias no tempo

$$
\begin{gathered}
\theta_{(\ell)}=0, \\
\theta_{(n)}<0 .
\end{gathered}
$$

Os horizontes aparentes estão definidos quase-localmente, o que significa [37] que a sua 
existência pode ser confirmada por medições em tempos finitos sem prévio conhecimento da estrutura causal do espaço-tempo. A definição de horizonte aparente, apesar de ser expressa em termos de quantidades escalares (expansões), depende da foliação escolhida para o espaçotempo [39]. Os campos nulos são ortogonais às hipersuperfícies, e portanto as expansões diferem em geral com a escolha da foliação [37]. A definição de Hayward dos trapping horizons [38] usa uma foliação particular do espaço-tempo, uma dupla foliação nula (sempre possível de construir, ao menos localmente [40]) e além de impor condições sobre as expansões, impõe condições sobre a derivada das expansões.

No capítulo 5 abordamos o problema de achar os horizontes aparentes de uma métrica de tipo McVittie generalizada (apresentada no capítulo 4). Usamos como uma guia a definição por Faraoni apresentada. 



\section{Capítulo 4}

\section{Geometrias de McVittie}

Em 1933 George C. McVittie encontrou uma solução exata das equações de Einstein com constante cosmológica (2.64), para um sistema físico que ele considerou como sendo um objeto esfericamente simétrico fixo na origem das coordenadas, de massa $m$ constante, imerso em um fluido em expansão com um fator de escala $a(t)$ e curvatura global do espaço-tempo $k$ [11]. O elemento de linha da métrica de McVittie é dado por

$$
d s^{2}=-\left[\frac{1-\frac{m}{2 a r}\left(1+\frac{k}{4} r^{2}\right)^{1 / 2}}{1+\frac{m}{2 a r}\left(1+\frac{k}{4} r^{2}\right)^{1 / 2}}\right]^{2} d t^{2}+\frac{\left[1+\frac{m}{2 a r}\left(1+\frac{k}{4} r^{2}\right)^{1 / 2}\right]^{4}}{\left(1+\frac{k}{4} r^{2}\right)^{2}} a^{2}\left(d r^{2}+r^{2} d \Omega^{2}\right)
$$

McVittie obteve (4.1) usando apenas umas poucas suposições. Começou com a isotropia em cada ponto e simetria esférica do espaço-tempo, depois assumiu homogeneidade excetuando na singularidade correspondente ao objeto de massa $m$ (a palavra inomogeneidade é também usada para se referir ao objeto central considerado). Com isto, não existe fluxo líquido do fluido afastando-se ou aproximando-se do objeto central, o qual implica pressão isotrópica do fluido [11]. De fato, um fluido compatível com essas propriedades é um fluido perfeito. 


\subsection{Limite $a(t) \rightarrow a_{0}, k \rightarrow 0$ (Schwarzschild)}

Para ver porque $m$ pode ser interpretado como a massa, consideremos o caso limite em que $a(t) \rightarrow a_{0}, k \rightarrow 0$. Com a troca de variáveis $\bar{r}=a_{0} r$ o elemento de linha (4.1) se torna,

$$
d s^{2}=-\left(\frac{1-\frac{m}{2 \bar{r}}}{1+\frac{m}{2 \bar{r}}}\right)^{2} d t^{2}+\left(1+\frac{m}{2 \bar{r}}\right)^{4}\left(d \bar{r}^{2}+\bar{r}^{2} d \Omega^{2}\right)
$$

que é a métrica de Schwarzschild em coordenadas isotrópicas (3.10). Como já vimos no capítulo 3 , a constante $m$ é a massa do objeto central.

\subsection{Limite $m \rightarrow 0$ (FLRW)}

Por outro lado, no limite em que a massa é nula, $m \rightarrow 0$, o elemento de linha (4.1) se escreve como

$$
d s^{2}=-d t^{2}+\frac{a^{2}}{\left(1-\frac{k}{4} r^{2}\right)^{2}}\left(d r^{2}+r^{2} d \Omega^{2}\right)
$$

que é o elemento de linha de Friedmann-Lamaître-Robertson-Walker [34] expresso em coordenadas isotrópicas. Sendo $m=0$, a inomogeneidade central inicial desaparece, a métrica de FLRW descreve um espaço-tempo cujas fatias a $t$ constante são superfícies tipo espaço homogêneas e isotrópicas [21, 24, 34]. Logo, existem seis campos vetorias de Killing tipo espaço associados à invariância da métrica sob translações e rotações e o espaço descrito é maximalmente simétrico e tem curvatura constante [24, 33].

A homogeneidade e a isotropia do espaço são exigências impostas quando se quer modelar o universo em grande escala. Baseado na observação, o universo é o mesmo (aproximadamente) quando enxergado em qualquer direção [33]. Por outro lado, o princípio Copernicano diz que não estamos em um lugar especial do universo e a homogeneidade a grande escala é geralmente assumida [21]. A métrica de FLRW (4.3) é usada como base na descrição da geometria em grande escala do universo.

A função $a=a(t)$ é um fator de escala que pode ser incluído na construção da métrica (4.3) e em geral depende da coordenada $t$. A função $a$ está relacionada com a evolução da geometria, em especial com a medida de distâncias espaciais. O parâmetro $k$ é a curvatura do espaço e pode ser positiva, negativa ou nula. 
Uma das formas usuais de escrever o elemento de linha (4.3) resulta da mudança seguinte

$$
\begin{aligned}
& \frac{d r^{2}}{\left(1+\frac{k}{4} r^{2}\right)^{2}}=\frac{d \bar{r}^{2}}{\lambda(\bar{r})} \\
& \frac{r^{2}}{\left(1+\frac{k}{4} r^{2}\right)^{2}}=\bar{r}^{2}
\end{aligned}
$$

onde obtém-se que $\lambda(\bar{r})=1-k \bar{r}^{2}$ e com a qual (4.3) pode ser expresso nas coordenadas comóveis [21, 37] segundo

$$
d s^{2}=-d t^{2}+a^{2}\left(\frac{d \bar{r}^{2}}{1-k \bar{r}^{2}}+\bar{r}^{2} d \Omega^{2}\right)
$$

Uma transformação de escala sobre $k$ com uma constante positiva equivale a uma transformação de escala sobre a parte espacial da métrica. Isto pode ser usado para definir um novo parâmetro $K$ que apenas toma os valores $0,1,-1$ e representa em cada caso uma das geometrias maximalmente simétrica para os slides de $t$ constante, plana, esférica e hiperbólica respectivamente [21].

\subsubsection{Distâncias e parâmetro de Hubble}

Consideremos agora a equação geodésica ${ }^{1}$ para a coordenada $t$ da métrica (4.6)

$$
\frac{d^{2} t}{d \lambda^{2}}+a \frac{d a}{d t}\left[\frac{1}{1-k \bar{r}^{2}}\left(\frac{d \bar{r}}{d \lambda}\right)^{2}+\bar{r}^{2}\left(\frac{d \theta}{d \lambda}\right)^{2}+\bar{r}^{2} \sin ^{2} \theta\left(\frac{d \phi}{d \lambda}\right)\right]=0
$$

Um observador $A$ fixo no espaço tem coordenadas $\left(t, \bar{r}_{0}, \theta_{0}, \phi_{0}\right)$ e a sua equação geodésica parametrizada com o tempo próprio é portanto

$$
\frac{d^{2} t}{d \tau^{2}}=0
$$

Com isto, vemos que $t$ é um parâmetro afim $t=\alpha \tau+\beta$. Aliás qualquer observador que possua coordenadas espaciais constantes é descrito por uma geodésica com o mesmo $t$. Razão pela qual as coordenadas são chamadas de comóveis e o tempo $t$ de tempo universal [34]. O espaço-tempo pode ser fatiado em fatias de $t$ constante.

\footnotetext{
${ }^{1}$ As equações geodésicas podem ser obtidas por meio das equações de Euler-Lagrange usando a lagrangiana $L=\frac{1}{2} g_{\mu \nu} \frac{d x^{\mu}}{d \lambda} \frac{d x^{\nu}}{d \lambda}[21]$
} 
A separação espacial (no tempo $t_{0}$ ) entre o observador $A$ e um objeto $B$ com coordenadas $\left(t_{0}, \bar{r}, \theta, \phi\right)$ consegue-se fazendo $t=t_{0}$ na métrica (4.6) e integrando

$$
\bar{R}\left(t_{0}\right)=\int_{0}^{l\left(t_{0}\right)} a\left(t_{0}\right)\left(\frac{d \bar{r}^{2}}{1-k \bar{r}^{2}}+\bar{r}^{2} d \Omega^{2}\right)^{1 / 2}=\int_{0}^{l\left(t_{0}\right)} a\left(t_{0}\right) d l\left(t_{0}\right)=a\left(t_{0}\right) l\left(t_{0}\right) .
$$

Esta expressão é válida para qualquer valor do tempo $t_{0}$, logo, (4.9) pode ser promovido a uma função de $t$

$$
\bar{R}(t)=a(t) l(t)
$$

o observador $A$ calcula a rapidez do objeto $B$ derivando em relação ao seu tempo $t$ a distância $\bar{R}(t)$, ou seja,

$$
\frac{d \bar{R}(t)}{d t}=\frac{d a(t)}{d t} l(t)+a(t) \frac{d l(t)}{d t}=\frac{\dot{a}(t)}{a(t)} \bar{R}(t)+a(t) \frac{d l(t)}{d t}
$$

onde se fez uso na última igualdade da distância (4.10) para o primeiro termo. Se define o parâmetro de Hubble o qual se associa com a velocidade de expansão do universo [33] como

$$
H(t)=\frac{\dot{a}}{a}
$$

O primeiro termo de (4.11) é chamado de velocidade de recessão $V_{\text {res }}$ [21] e em termos do parâmetro de Hubble (4.12) se escreve

$$
V_{\text {res }}(t)=H(t) R(t)
$$

Se avaliarmos $t=t_{0}$ sendo $t_{0}$ o tempo correspondente a hoje, a equação (4.13) expressa que a velocidade com que se afasta hoje um objeto em relação a um observador é proporcional à distância que os separa hoje. Note-se que na observação de um objeto celeste, a informação recebida em $t_{0}$ foi emitida algum tempo atrás diferente de $t_{0}$.

Na equação (4.11), o último termo é chamado de velocidade peculiar $V_{\text {pec }}$ [21]. Se o objeto $B$ permanecer em coordenadas espaciais fixas $V_{p e c}=0$. Pode ser mostrado que $V_{p e c}$ é sempre menor que a velocidade da luz no entanto que $V_{\text {res }}$ não necessariamente [21].

O parâmetro de Hubble é uma função importante na descrição da dinâmica do universo assim como uma ferramenta útil para calcular distâncias e tempos cósmicos [41]. É possível obter analiticamente o parâmetro de Hubble e o fator de escala em situações especiais. 


\subsubsection{Fluidos perfeitos e as equações de Friedmann}

A homogeneidade e isotropia espacial presente na métrica de FLRW estende-se ao tensor de energia-momento $T_{\mu \nu}$ por meio das equações de Einstein. O tensor resultante pode ser entendido como descrevendo um fluido perfeito, definido como um fluido caracterizado por ter densidade de energia $\rho$, pressão $p$ e 4-velocidade $\boldsymbol{U}$ em cada ponto, tal que a pressão é isotrópica no referencial comóvel de cada elemento do fluido [42]. Tensão de cisalhamento, anisotropias na pressão, viscosidade ou transferências de calor estão ausentes na descrição de fluido perfeito. Uma consequência da isotropia é que o $T_{\mu \nu}$ é diagonal no referencial comóvel [24]. No sistema comóvel o tensor de energia-momento pode ser representado matricialmente como

$$
\tilde{T}^{\mu \nu}=\left(\begin{array}{cccc}
\rho & 0 & 0 & 0 \\
0 & p & 0 & 0 \\
0 & 0 & p & 0 \\
0 & 0 & 0 & p
\end{array}\right)
$$

e a forma covariante do tensor de energia-momento do fluido perfeito é dada por

$$
T^{\mu \nu}=(\rho+p) U^{\mu} U^{\nu}+p g^{\mu \nu}
$$

Adicionalmente, $\rho$ e $p$ estão relacionados por uma equação de estado que descreve o tipo de fluido perfeito particular sob consideração [34]. A equação de estado barotrópica da forma

$$
p=\omega \rho,
$$

descreve os principais tipos de fluido usados em cosmologia [41]. Por exemplo, $\omega=0$ descreve uma poeira de partículas, $\omega=1 / 3$ fótons, $\omega=-1$ energia escura.

A substituição direta da métrica (4.6) nas equações de Einstein com constante cosmológica (2.64) e tensor de energia-momento dado por (4.15) produz as duas primeiras equações do conjunto seguinte e a terceira é a equação de continuidade para a densidade de energia e resulta 
da conservação do tensor de energia-momento

$$
\begin{aligned}
H^{2} & =\left(\frac{\dot{a}}{a}\right)^{2}=\frac{8 \pi}{3} \rho-\frac{k}{a^{2}}+\frac{\Lambda}{3}, \\
\frac{\ddot{a}}{a} & =-\frac{4 \pi}{3}(\rho+3 p)+\frac{\Lambda}{3}, \\
\dot{\rho} & +3(\rho+p) H=0 .
\end{aligned}
$$

As equações (4.17), (4.18) e (4.19) são conhecidas como equações de Friedmann-Lamaître ou equações de Friedmann [21] e são usadas junto com a equação de estado (4.16) como a base dos modelos cosmológicos FLRW que têm por objetivo principal encontrar a forma do fator de escala $a$ ou o parâmetro de Hubble $H$ além da pressão e densidade de forma explícita.

Soluções em diversos ambientes cosmológicos e épocas do universo são encontradas na literatura [34, 42, 30, 21]. Temos interesse em soluções nas quais a curvatura $k$ é nula. Pode ser substituída a equação de estado (4.16) na equação de continuidade para obter uma expressão de $\rho$ em termos de $a$

$$
\rho=\rho_{0}\left(\frac{a_{0}}{a}\right)^{3(1+\omega)} .
$$

Se observa que a densidade diverge quando $a$ for zero o que é característico da singularidade de tipo big bang [41]. Se a densidade dada por (4.20) é substituída na equação de Friedmann (4.17) na qual $k=0$, e a integração da equação for feita com a condição que em $t=0$ o fator de escala $a(0)=0$ obtemos [33],

$$
a^{3(1+\omega)}=\frac{4 \pi \rho_{0}}{\Lambda} \cosh [(1+\omega) \sqrt{3 \Lambda} t]-\frac{4 \pi \rho_{0}}{\Lambda},
$$

onde $\omega \neq-1$. Por sua vez, (4.21) é usado para encontrar o parâmetro de Hubble (4.12) dando como resultado

$$
H_{\omega}(t)=\sqrt{\frac{\Lambda}{3}} \frac{\sinh [(1+\omega) \sqrt{3 \Lambda} t]}{\cosh [(1+\omega) \sqrt{3 \Lambda} t]-1} .
$$

Nota-se o comportamento assintótico seguinte

$$
H_{\omega}(t)= \begin{cases}\sqrt{\frac{\Lambda}{3}} \equiv H_{0} & \text { se } \quad t \gg \frac{1}{\sqrt{\Lambda}} \\ \frac{2}{3(1+\omega) t} & \text { se } t \ll \frac{1}{\sqrt{\Lambda}}\end{cases}
$$


Pode ser mostrado $[34,41]$ que $H_{\omega=-1}(t)=H_{0}$ e que se $\Lambda=H_{0}=0$ temos $H_{\omega \neq-1}=\frac{2}{3(1+\omega) t}$.

Vemos que o elemento de linha de McVittie parece ser uma boa descrição para um corpo esfericamente simétrico de massa $m$ em um universo em expansão. Porém, a consideração de (4.1) e a sua validade na representação de uma situação física real tem sido razão de debate durante décadas, começando pelo fato que o fluido preenchendo o universo tem densidade de energia uniforme mas pressão não uniforme [12].

\subsection{Métrica de McVittie generalizada}

No contexto do modelo cosmológico padrão $\Lambda$-CDM, recentes observações independentes da distribuição espectral das anisotropias da radiação cósmica de fundo $(C M B)$, medidas envolvendo lentes gravitacionais e $C M B$, e a análise das oscilações acústicas de bárions $(B A O)$ [43], sugerem que o universo é espacialmente plano $(k=0)$. Desta forma, temos interesse na generalização de métricas de tipo (4.1) $\operatorname{com} k=0$,

$$
d s^{2}=-\left[\frac{1-\frac{m}{2 a(t) r}}{1+\frac{m}{2 a(t) r}}\right]^{2} d t^{2}+\left[1+\frac{m}{2 a(t) r}\right]^{4} a(t)^{2}\left(d r^{2}+r^{2} d \Omega^{2}\right) .
$$

O elemento de linha (4.24) é denominado as vezes elemento de linha ou métrica de McVittie, apesar de ter a variante mais geral (4.1) [37, 20]. Existe uma singularidade em (4.24) sobre a 2-esfera $r=m / 2 a$, que se reduz ao horizonte de Schwarzschild no caso $a=1$. Isto pode ser comprovado com o cálculo do escalar de Ricci [44] nesse valor de $r$. Além disso, essa é uma singularidade tipo espaço [37] no passado causal se $\dot{a}>0$ [20]. Por outro lado, o espaço-tempo obtido a partir da métrica de McVittie (4.24) tem um futuro infinito nulo ou tipo espaço a $r$ e $t$ grandes de tipo FLRW [20].

Em [20] se propõe uma generalização de (4.24) que consiste em permitir que a massa do objeto central varie com o tempo. Isto significa considerar acreção no sistema. O elemento de linha vai ser dado por

$$
d s^{2}=-\left[\frac{1-\frac{m(t)}{2 a(t) r}}{1+\frac{m(t)}{2 a(t) r}}\right]^{2} d t^{2}+\left[1+\frac{m(t)}{2 a(t) r}\right]^{4} a(t)^{2}\left(d r^{2}+r^{2} d \Omega^{2}\right)
$$


A métrica (4.25) é uma solução das equações de Einstein (2.64) quando se abandona a ideia de ter um fluido perfeito como fonte de campo. No caso em que o fluido considerado é imperfeito, a solução é exata ficando algumas funções livres para serem escolhidas [20] (ver apêndice C).

O modelo de fluido imperfeito é útil quando existem variações importantes na pressão, densidade e velocidade localmente e não está bem definido um estado de equilíbrio térmico no fluido e a energia é dissipada na forma de calor [30]. O tensor de energia-momento associado pode ser expresso na forma

$$
\mathcal{T}^{\mu \nu}=T^{\mu \nu}-\eta H^{\mu \sigma} H^{\nu \lambda} W_{\sigma \lambda}-\chi\left(H^{\mu \sigma} U^{\nu}+H^{\nu \sigma} U^{\mu}\right) Q_{\sigma}-\zeta H^{\mu \nu} \nabla_{\sigma} U^{\sigma}
$$

onde $T^{\mu \nu}$ é o tensor de energia-momento para o fluido perfeito dado por (4.15). Se identificam $\chi, \eta$ e $\zeta$ como os coeficientes de condução de calor, viscosidade de cisalhamento e viscosidade volumar respectivamente. Por outro lado, o vetor $U^{\mu}$ é a velocidade do transporte de partículas [30]. E os tensores

$$
\begin{aligned}
W_{\mu \nu} & =\nabla_{\nu} U_{\mu}+\nabla_{\mu} U_{\nu}-\frac{2}{3} g_{\mu \nu} \nabla_{\sigma} U^{\sigma}, \\
Q_{\mu} & =\nabla_{\mu} T+T U^{\nu} \nabla_{\nu} U_{\mu}, \\
H_{\mu \nu} & =g_{\mu \nu}+U_{\mu} U_{\nu},
\end{aligned}
$$

onde $T$ é a temperatura, são o tensor de cisalhamento, vetor de fluxo de calor e por último um tensor de projeção sobre o plano ortogonal a $U^{\mu}$. A simetria esférica da métrica de McVittie generalizada implica que a viscosidade de cisalhamento é nula [20]. O tensor de energia-momento mais geral no nosso caso vai ser

$$
\mathcal{T}^{\mu \nu}=T^{\mu \nu}-\chi\left(H^{\mu \sigma} U^{\nu}+H^{\nu \sigma} U^{\mu}\right) Q_{\sigma}-\zeta H^{\mu \nu} \nabla_{\sigma} U^{\sigma}
$$

Será conveniente escrever o elemento de linha (4.25) em termos do raio areal $\hat{r}$ definido tal que superfícies tipo espaço a $t$ e $\hat{r}$ constantes têm área $4 \pi \hat{r}^{2}$. Para tal fim, fazemos uma mudança na coordenada radial que generaliza (3.9), ou seja,

$$
\hat{r}=a(t) r\left[1+\frac{m(t)}{2 a(t) r}\right] .
$$

A relação (4.31) define dois valores diferentes da nova coordenada $\hat{r}$ para cada valor de $r$ igual 
que no caso independente do tempo (discutido na seção 3.1). Existem assim dois ramos e escolhemos, acompanhando a [20], aquele que mapeia desde $r=m / 2 a$ em $\hat{r}=2 m$ até $r \rightarrow \infty$ em $\hat{r} \rightarrow \infty$. Com essa troca, escrevemos a 1-forma $d r$ em termos de $d \hat{r}$ e $d t$ como

$$
d r=\frac{d \hat{r}}{a\left(1+\frac{m}{2 a r}\right)\left(1-\frac{m}{2 a r}\right)}-\left[r a H+\frac{m M}{\left(1-\frac{m}{2 a r}\right)}\right] \frac{d t}{a},
$$

onde foi introduzido o parâmetro de Hubble (4.12) e a função $M$,

$$
M=M(t) \equiv \frac{\dot{m}}{m}
$$

Definimos também a função $R$, por meio da expressão

$$
R=R(t, \hat{r}) \equiv \sqrt{1-\frac{2 m(t)}{\hat{r}}}
$$

Por outro lado as relações a seguir, que podem ser obtidas por meio de manipulações algébricas de (4.31) serão úteis na escrita do elemento de linha em termos das novas coordenadas,

$$
\begin{aligned}
R^{2} & =\frac{\left(1-\frac{m}{2 a r}\right)^{2}}{\left(1+\frac{m}{2 a r}\right)^{2}}, \\
\frac{2}{1+R} & =\left(1+\frac{m}{2 a r}\right) .
\end{aligned}
$$

A métrica de McVittie generalizada (4.25) pode ser expresso em termos do raio areal $\hat{r}$ na forma

$$
d s^{2}=-R^{2} d t^{2}+\left[\frac{d \hat{r}}{R}-\tilde{H} \hat{r} d t\right]^{2}+\hat{r}^{2} d \Omega^{2}
$$

onde definimos uma nova função para simplificar a notação

$$
\tilde{H}=\tilde{H}(t, \hat{r}) \equiv H+\frac{2 m M}{\hat{r} R(1+R)}
$$

Ainda é possível fazer uma troca de variáveis fazendo com que o elemento de linha possa ser expresso na forma de Schwarzschild. Vemos que existe um termo isolado com $d t^{2}$ em (4.37). Podemos expandir o interior do colchete e rearranjá-lo para obter

$$
d s^{2}=-\left[\left(R^{2}-\tilde{H}^{2} \hat{r}^{2}\right) d t^{2}+\frac{2}{R} \tilde{H} \hat{r} d \hat{r} d t\right]+\frac{d \hat{r}^{2}}{R^{2}}+\hat{r}^{2} d \Omega^{2}
$$


Tirando o fator comum no termo que acompanha o $d t^{2}$ da expressão dentro dos colchetes, completando quadrados no interior e rearranjando obtém-se

$$
d s^{2}=-\left(R^{2}-\tilde{H}^{2} \hat{r}^{2}\right)\left[d t+\frac{\tilde{H} \hat{r} d \hat{r}}{R\left(R^{2}-\tilde{H}^{2} \hat{r}^{2}\right)}\right]^{2}+\frac{d \hat{r}^{2}}{\left(R^{2}-\tilde{H}^{2} \hat{r}^{2}\right)}+\hat{r}^{2} d \Omega^{2}
$$

Existe agora uma sugestão explícita para a nova coordenada. Definimos a coordenada temporal $T$ por meio da 1-forma (que em geral não é fechada)

$$
d T=d t+\frac{\tilde{H} \hat{r} d \hat{r}}{R\left(R^{2}-\tilde{H}^{2} \hat{r}^{2}\right)}
$$

e usando a definição (4.34) obtemos a forma de Schwarzschild para o elemento de linha de McVittie generalizado

$$
d s^{2}=-\left[1-\frac{2 m(t)}{\hat{r}}-\tilde{H}^{2}(t, \hat{r}) \hat{r}^{2}\right] d T^{2}+\frac{d \hat{r}^{2}}{\left[1-\frac{2 m(t)}{\hat{r}}-\tilde{H}^{2}(t, \hat{r}) \hat{r}^{2}\right]}+\hat{r}^{2} d \Omega^{2}
$$

A sugestão explícita para a troca de variáveis exposta em (4.40) pode ser mais geral do que a escolha (4.41), por exemplo poderíamos incluir uma função a priori desconhecida $F$ que poderia ser o fator integrante ${ }^{2}$ de (4.41), pode ser proposta a seguinte 1-forma que define a variável $\tilde{T}$

$$
d \tilde{T}=\frac{d T}{F} .
$$

Esta expressão gera um elemento de linha que não tem exatamente a forma de Schwarzschild (nas coordenadas usuais)

$$
d s^{2}=-\left[1-\frac{2 m(t)}{\hat{r}}-\tilde{H}^{2}(t, \hat{r}) \hat{r}^{2}\right] F^{2} d \tilde{T}^{2}+\frac{d \hat{r}^{2}}{\left[1-\frac{2 m(t)}{\hat{r}}-\tilde{H}^{2}(t, \hat{r}) \hat{r}^{2}\right]}+\hat{r}^{2} d \Omega^{2}
$$

Porém, nesse trabalho não temos razões para incluir a forma explícita de tais funções.

O elemento de linha (4.44) também pode ser pensado como um caso particular de generalização de métricas esfericamente simétricas de tipo (3.1) com uma função $f$ que agora depende também do tempo. No caso de (4.44), a dependência do $f$ com o tempo coordenado é implícita,

\footnotetext{
${ }^{2}$ A idéia foi tomada de [37] onde se faz exatamente mesma coisa para escrever o elemento de linha particular de McVittie na forma de Schwarzschild.
} 
e temos que

$$
f(t(T, \hat{r}), \hat{r})=f(t, \hat{r})=1-\frac{2 m(t)}{\hat{r}}-\tilde{H}^{2}(t, \hat{r}) \hat{r}^{2} .
$$

Substituindo a forma explícita da função $\tilde{H}$ (4.38), temos para o elemento de linha de McVittie generalizado

$$
f(t, \hat{r})=1-\frac{2 m(t)}{\hat{r}}-\left(H+\frac{2 m M}{\hat{r} R(1+R)}\right)^{2} \hat{r}^{2} .
$$

No caso em que não é considerada variação temporal da massa, a $f$ de McVittie $f_{M V}$ é dada por,

$$
f_{M V}(t, \hat{r})=1-\frac{2 m}{\hat{r}}-H^{2} \hat{r}^{2}
$$

onde $H$ ainda é uma função do tempo. A métrica de McVittie define horizontes aparentes dinâmicos no caso em que $H$ é uma função arbitrária do tempo [37], serão calculados numericamente no próximo capítulo.

O elemento de linha (4.42) quando $m$ não depende de $t$ (e portanto o elemento de linha (4.24)) no limite em que o fator de Hubble $H=\dot{a} / a \rightarrow H_{0}$ é uma constante, se torna o elemento de linha de Schwarzschild-de Sitter, caracterizado por uma $f$ da forma

$$
f_{S d S}(\hat{r})=1-\frac{2 m}{\hat{r}}-H_{0}^{2} \hat{r}^{2}
$$

sendo a mais simples das soluções das equações de Einstein com uma inomogeneidade central e constante cosmológica positiva. O espaço-tempo resultante é estático e assintoticamente de Sitter. De fato, tomar o limite $r \rightarrow \infty$ nos deixa com o elemento de linha (4.3) com $k=0$ e $a=a_{0} e^{H_{0} t}$ na forma de Schwarzschild. Interpreta-se fisicamente este caso como um corpo esfericamente simétrico em um fundo cosmológico de Sitter. Vemos então que a generalização da métrica de McVittie apresentada é também uma generalização da métrica de Schwarzschildde Sitter e inclusive dos espaços de Schwarzschild e de FLRW separadamente.

A métrica de McVittie generalizada na forma apresentada em (4.37) pode ser expressa na forma de Schwarzschild usando a 1-forma $d T$ dada por (4.41). Os casos limite dessa métrica incluem McVittie com curvatura espacial $k=0$, (4.24) (quando $m(t) \rightarrow m_{0}$ ), Schwarzschildde Sitter (quando $m(t) \rightarrow m_{0}$ e $H(t) \rightarrow H_{0}$ que por sua vez inclui Schwarzschild e de Sitter quando $H_{0} \rightarrow 0$ e $m_{0} \rightarrow 0$ respectivamente), FLRW (quando $m(t) \rightarrow 0$ ) e na forma de Schwarzschild é imediato verificar esses limites. 


\subsubsection{Expansões de congruências geodésicas nulas}

No capítulo 3 apresentamos várias formas em que as expansões de campos nulos geodésicos podem ser calculados. Usando (3.39) e (3.40), não precisamos da forma explícita dos campos vetoriais nulos mas precisamos do elemento de área da seção transversa às congruências de geodésicas nulas radiais. Vemos então a importância do raio areal no conjunto de coordenadas.

Vamos ver como seria o cálculo no caso de métricas tipo (4.44) as quais como vimos incluem McVittie generalizado, McVittie, Schwarszchild de Sitter, Schwarzschild, de Sitter, e inclusive outros casos como Reissner-Nordstrom. Consideremos a expressão da expansão de um campo nulo $\boldsymbol{V}(3.38)$,

$$
\theta_{(V)}=\frac{1}{\sqrt{h}} \mathcal{L}_{\boldsymbol{V}} \sqrt{h}=\frac{1}{\sqrt{h}} \frac{d}{d \lambda_{\boldsymbol{V}}} \sqrt{h},
$$

onde a métrica induzida tem a ver com a parte angular do elemento de linha (4.44),

$$
\begin{aligned}
d s^{2} & =-f(t, \hat{r}) F^{2} d \tilde{T}^{2}+f(t, \hat{r})^{-1} d \hat{r}^{2}+\hat{r}^{2} d \theta^{2}+\hat{r}^{2} \sin ^{2} \theta d \phi^{2} \\
& =-f(t, \hat{r}) F^{2} d \tilde{T}^{2}+f(t, \hat{r})^{-1} d \hat{r}^{2}+h_{\alpha \beta} d x^{\alpha} d x^{\beta}
\end{aligned}
$$

A derivada em relação ao parâmetro $\lambda_{V}$ significa derivação em relação aos parâmetros que definem geodésicas nulas ingoing ou outgoing, dependendo do qual que é o campo nulo $\boldsymbol{V}$ nesse sistema de coordenadas. Substituindo $h$ e com o uso da regra da cadeia podemos escrever a (4.49) como

$$
\theta_{(V)}=\frac{1}{\hat{r}^{2}} \frac{d \hat{r}^{2}}{d \lambda_{\boldsymbol{V}}}=\left.\frac{2}{\hat{r}} \frac{d \hat{r}}{d \tilde{T}}\right|_{ \pm} \frac{d \tilde{T}}{d \lambda_{\boldsymbol{V}}}
$$

em que foi enfatizado que a velocidade coordenada $d \hat{r} / d \tilde{T}$ deve ser avaliada sobre a geodésica nula ingoing (-) ou outgoing $(+)$. A expressão para essa velocidade coordenada pode ser obtida impondo-se que o elemento de linha (4.44) $\operatorname{com} \theta=\theta_{0}$ e $\phi=\phi_{0}$ seja nulo. Com isto,

$$
\left.\frac{d \hat{r}}{d \tilde{T}}\right|_{ \pm}= \pm f(t, \hat{r}) F
$$

Por outro lado, a derivada $d \tilde{T} / d \lambda_{\boldsymbol{V}}$ corresponde à componente zero $V^{0}$ do vetor tangente à geodésica nula considerada e que pode ser obtida integrando as equações geodésicas. A expansão de $\boldsymbol{V}$ pode ser expressa como

$$
\theta_{(V)}= \pm \frac{2}{\hat{r}} f(t, \hat{r}) F V^{0}
$$


É possível mostrar que $F V^{0}=F d \tilde{T} / d \lambda_{V}>0$. Com tais fins, consideremos a definição da 1-forma $d \tilde{T}$ dada por (4.43) da qual decorre que

$$
V^{0}=\frac{d \tilde{T}}{d \lambda_{V}}=\frac{1}{F} \frac{d T}{d \lambda_{V}}
$$

e por outro lado, da expressão (4.41) que define a 1-forma $d T$, segue para o campo geodésico nulo radial $\boldsymbol{V}$ que

$$
F V^{0}=\frac{d T}{d \lambda_{\boldsymbol{V}}}=\frac{d t}{d \lambda_{\boldsymbol{V}}}\left(1+\left.\frac{\tilde{H} \hat{r}}{R\left(R^{2}-\tilde{H}^{2} \hat{r}^{2}\right)} \frac{d \hat{r}}{d t}\right|_{ \pm}\right)
$$

onde aparece a velocidade coordenada $d \hat{r} /\left.d t\right|_{ \pm}$que pode ser obtida substituindo $d s^{2}=0$ (com $\theta$ e $\phi$ fixos) na métrica de McVittie generalizada (4.37),

$$
\left.\frac{d \hat{r}}{d t}\right|_{ \pm}=R(\tilde{H} \hat{r} \pm R)
$$

Agora usando a expressão (4.56) em (4.55), temos

$$
F V^{0}=\frac{d T}{d \lambda_{\boldsymbol{V}}}=\frac{d t}{d \lambda_{\boldsymbol{V}}}\left(1+\frac{\tilde{H} \hat{r}(\tilde{H} \hat{r} \pm R)}{(R+\tilde{H} \hat{r})(R-\tilde{H} \hat{r})}\right)
$$

O sinal positivo corresponde ao campo $V=\ell$ (geodésicas outgoing), então a expressão (4.57) torna-se

$$
F \ell^{0}=\frac{d T}{d \lambda_{\ell}}=\frac{d t}{d \lambda_{\ell}}\left(1+\frac{\tilde{H} \hat{r}}{(R-\tilde{H} \hat{r})}\right) .
$$

Se assumirmos que $d t / d \lambda_{\ell}>0$, também $F \ell^{0}>0$ desde que $(R-\tilde{H} \hat{r})>0$ o qual implica $f>0$.

O sinal negativo corresponde ao campo $\boldsymbol{V}=\boldsymbol{n}$ (geodésicas ingoing), então a expressão (4.57) torna-se

$$
F n^{0}=\frac{d T}{d \lambda_{n}}=\frac{d t}{d \lambda_{n}}\left(1-\frac{\tilde{H} \hat{r}}{(R+\tilde{H} \hat{r})}\right) .
$$

De forma semelhante, se assumirmos que $d t / d \lambda_{n}>0$, também $F n^{0}>0$ desde que $f=$ $(R-\tilde{H} \hat{r})(R+\tilde{H} \hat{r})>0$.

Isto significa, que se o tempo coordenado $t$ aumentar com o avanço dos feixes de luz (é 
possível escolher tal orientação), então o tempo coordenado $\tilde{T}$ também aumenta com o avanço dos feixes de luz. Aliás, as expansões serão nulas só se $f$ for nulo. A condição para a existência de horizontes aparentes se torna

$$
\theta_{(V)}= \pm \frac{2}{\hat{r}} f(t, \hat{r}) F V^{0}=0 \Rightarrow f(t, \hat{r})=0
$$

No caso Schwarzschild, a transformação de coordenadas (4.43) não envolve a coordenada radial e a função $F$ pode ser escolhida como a unidade. Aliás, $f$ não depende de $t$ e podemos obter explicitamente os campos vetoriais nulos $\boldsymbol{\ell}$ e $\boldsymbol{n}$. Para isto, considere-se a Lagrangiana para a métrica de Schwarzschild e o campo $\boldsymbol{V}=\left(V^{0}, V^{1}, 0,0\right)$,

$$
L=\frac{1}{2} g_{\mu \nu} V^{\mu} V^{\nu}
$$

a métrica de Schwarzschild é estática, por tanto a primeira das equações geodésicas é

$$
\frac{d}{d \lambda_{\boldsymbol{V}}} \frac{\partial L}{\partial \dot{t}}=-V^{1}\left(f \frac{d V^{0}}{d r}+\frac{d f}{d r} V^{0}\right)=0
$$

a condição de que $\boldsymbol{V}$ seja um campo nulo é suficiente para obter $V^{1}$ em termos de $V^{0}$,

$$
V^{1}= \pm f V^{0}
$$

substituindo em (4.62), obtemos

$$
f V^{0}\left(f \frac{d V^{0}}{d r}+\frac{d f}{d r} V^{0}\right)=0
$$

Se $f$ for diferente de zero, o campo $\boldsymbol{V}$ solução vai ser dado por

$$
\boldsymbol{V}=\left(\frac{A}{f}, \pm A\right)
$$

para alguma constante $A$. O sinal (-) correspondendo a geodésicas ingoing (campo $\boldsymbol{n}$ ) e o sinal $(+)$ às outgoing (campo $\ell$ ). A constante $A$ pode ser escolhida como a unidade. Nesse caso as 
expansões são

$$
\begin{aligned}
\theta_{(\ell)} & =\frac{2}{r} f \ell^{0}=\frac{2}{r}, \\
\theta_{(\boldsymbol{n})} & =-\frac{2}{r} f n^{0}=-\frac{2}{r} .
\end{aligned}
$$

Vemos que ambas as expansões não se anulam quando $f$ for diferente de zero. Então a única forma de anular a expansão de congruências geodésicas nulas nesse sistema de coordenadas é com a condição

$$
g_{00}=-f=0,
$$

que no caso Schwarzschild acontece quando $r=2 m$. Se a métrica for (4.44) a condição para ter horizontes aparentes vai ser também (4.68).

As expansões das congruências nulas também podem ser obtidas para a métrica de McVittie generalizada na forma (4.37). Nesse caso a expressão das expansões vai ser semelhante a (4.51),

$$
\theta_{(V)}=\left.\frac{2}{\hat{r}} \frac{d \hat{r}}{d t}\right|_{ \pm} \frac{d t}{d \lambda_{V}}
$$

A expressão para a velocidade coordenada de geodésicas radiais é dada por (4.56) e denotando $d t / d \lambda_{\boldsymbol{V}}=\tilde{V}^{0}$ a expressão das expansões se torna

$$
\theta_{(V)}=\frac{2}{\hat{r}} R(\tilde{H} \hat{r} \pm R) \tilde{V}^{0}
$$

Sendo $R$ (4.34) positivo na região de interesse, e sendo o parâmetro de Hubble $H$ positivo para um universo em expansão, temos que a condição para ter horizontes aparentes é dada pela condição $\theta_{(\boldsymbol{n})}=0$, ou seja com a escolha de sinal negativo em (4.70) e portanto temos

$$
\tilde{H} \hat{r}-R=0 .
$$

Podemos notar que a função $f$ pode ser fatorizada,

$$
f(t, \hat{r})=R^{2}-\tilde{H}^{2} \hat{r}^{2}=(R+\tilde{H} \hat{r})(R-\tilde{H} \hat{r})
$$

de forma que a condição para ter horizontes aparentes é igual em ambos sistemas de coordena- 
das. Usando a definição (4.34), podemos expressar $\tilde{H}$ como

$$
\tilde{H}=H-M+\frac{M}{R} .
$$

A condição (4.71) pode ser escrita na forma

$$
R(\hat{r} H-R)+\hat{r} M(1-R)=0 .
$$

Quando não se tem acreção de massa, $M=0$ e a equação (4.74) se reduz a

$$
1-\frac{2 m}{\hat{r}}-H^{2} \hat{r}^{2}=f_{M V}(t, \hat{r})=0
$$

que é a condição para ter horizontes no caso McVittie. A equação (4.74) pode ser expressa na forma de um polinômio de grau 4 na variável $\hat{r}$. No próximo capítulo apresentamos as soluções das equações (4.74) e (4.75). 


\section{Capítulo 5}

\section{Buracos negros cosmológicos}

Procuramos nesse capítulo horizontes aparentes em uma geometria determinada por funções $H$ e $m$ que podem estar associadas com idealizações de sistemas físicos reais. Primeiro, a condição que determina a existência dos horizontes aparentes (4.74) pode ser expressa em termos do raio areal $\hat{r}$ ao substituir $R$ pela definição (4.34). Rearranjando, obtemos

$$
H(H-2 M) \hat{r}^{4}-2\left[m(H-M)^{2}-M\right] \hat{r}^{3}-(4 m M+1) \hat{r}^{2}+4 m \hat{r}-4 m^{2}=0,
$$

em que cada um dos termos $\hat{r}, m, M$ e $H$ são funções de $t$. Para cada valor fixo de $t$, essa equação pode ser considerada um polinômio de grau quatro na variável $\hat{r}=\hat{r}(t)$. Dado um intervalo na variável $t$ e um conjunto de pontos no intervalo em que os parâmetros estejam bem definidos, podemos obter as raízes $\hat{r}$ para cada valor de $t$. São então escolhidas raízes específicas usando o critério que aquelas válidas fossem reais e consistentes com o intervalo em que $\hat{r}$ está definido.

Se considera uma família de funções de massa baseada na escolha em [20]. Se inclui um conjunto de parâmetros que permite modificar escalas, conforme a expressão

$$
m^{*}\left(t, t_{1}, t_{2}, m_{0}, s\right)=\left\{\begin{array}{lcc}
m_{0} & \text { se } & 0<t \leq t_{1}, \\
m_{0}+s\left[1-\cos \left(\frac{\pi\left(t-t_{1}\right)}{t_{2}-t_{1}}\right)\right] & \text { se } t_{1}<t \leq t_{2}, \\
m_{0}+2 s & \text { se } \quad t_{2}<t .
\end{array}\right.
$$

Em (5.2) o parâmetro $s$ representa um gap do valor de massa inicial $m_{0}$ e a conexão é feita por meio de uma função senoidal que suaviza a mudança. Os parâmetros $t_{1}$ e $t_{2}$ são usados 


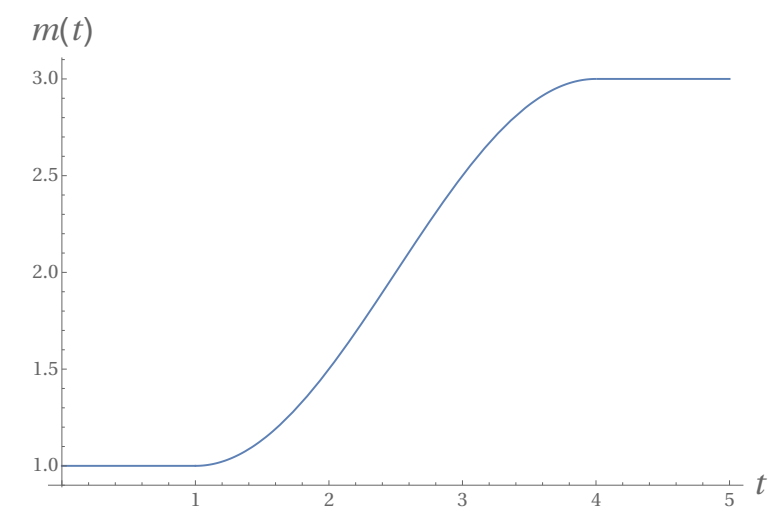

Figura 5.1: Uma função $m(t)$ particular resulta da escolha de valores particulares nos parâmetros $m_{0}, s, t_{1}$ e $t_{2}$ em (5.2). Neste gráfico temos $m(t)=m^{*}(t, 1,4,1,1)$.

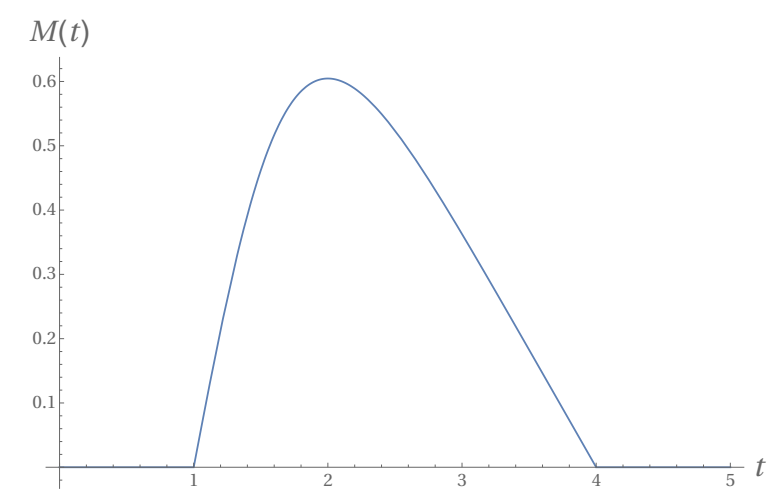

Figura 5.2: A correspondente função $M(t)$ associada à $m(t)$ da figura 5.1 resulta da escolha de valores particulares nos parâmetros $m_{0}, s, t_{1}$ e $t_{2}$ em (5.3). Neste gráfico temos $M(t)=M^{*}(t, 1,4,1,1)$.

também para controlar a mudança de massa e inclusive para localizar a acreção de massa em algum intervalo específico de $t$. As grandezas mencionadas se assumem terem as dimensões adequadas para serem consistentes. O gráfico de uma possível função massa é apresentado na figura 5.1. Lembrando que a função $M(t)=\dot{m}(t) / m(t)$ depende da função de massa, podemos construir uma família

$$
M^{*}\left(t, t_{1}, t_{2}, m_{0}, s\right)=\frac{1}{m^{*}} \frac{\partial m^{*}}{\partial t} .
$$

O correspondente $M(t)$ da $m(t)$ na figura 5.1 é mostrado no gráfico 5.2. Igualmente se considera uma família de parâmetros de Hubble baseado na escolha em [20], que pode-se justificar em base à análise assintótica apresentada na seção 4.2.2. São assim considerados modelos diferentes da evolução do universo na nossa descrição,

$$
H^{*}\left(t, \sigma, H_{0}\right)=\frac{2 \sigma}{3 t}+H_{0}
$$


Com isto justificamos a terminologia doravante usada para referirmos a elementos particulares das famílias $M^{*}$ e $H^{*}$ em que faremos referência apenas aos parâmetros que os definem.

\subsection{Caso $s=0, \sigma=0$ (Schwarszchild-de Sitter)}

A equação (5.1) se reduz em uma equação mais simples no caso em que $s=0$ (que define a massa constante) e $\sigma=0$ (que define o parâmetro de Hubble constante),

$$
-H_{0}^{2} \hat{r}^{4}+2 m_{0} H_{0}^{2} \hat{r}^{3}+\hat{r}^{2}-4 m_{0} \hat{r}+4 m_{0}^{2}=0
$$

Agora (5.5) pode ser reduzida a um polinômio de terceiro grau usando a condição implícita na definição da coordenada $\hat{r}$ que $\hat{r}(t)>2 m_{0}$. Aliás, como vimos no capítulo 4 , quando $m$ e $H$ forem independentes do tempo o espaço-tempo é Schwarzschild-de Sitter, $f(t, \hat{r})=f_{S d S}(\hat{r})$. As soluções da equação (5.5) são bem conhecidas, mas nem sempre existem soluções reais e positivas. Em particular, em [45] é apresentada a condição para ter horizontes

$$
m_{0} H_{0} \leq \frac{1}{3 \sqrt{3}}
$$

sendo $m_{0}$ e $H_{0}$ positivos. As soluções foram encontradas numericamente e são apresentadas na figura 5.3. Um dos horizontes aparentes resultantes coincide com o horizonte de Schwarzschild $\hat{r}=2 m_{0}$ no caso que $H_{0} \rightarrow 0$. O outro coincide com o horizonte cosmológico da métrica de Sitter $\hat{r}=1 / H_{0}$ no caso que $m_{0} \rightarrow 0$. No espaço-tempo de Schwarzschild-de Sitter os horizontes aparentes coincidem com os horizontes de eventos. Embora isso não seja sempre verdade, neste caso isso acontece porque o espaço-tempo é estático [37].

A negação da desigualdade (5.6) leva a soluções complexas de (5.5) e pode ser interpretada como a impossibilidade de que o horizonte de buraco negro seja maior do que horizonte cosmológico [37]. No caso extremo, em que $m_{0} H_{0}=1 / 3 \sqrt{3}$, os horizontes coincidem conforme à figura $5.3(a)$. Se observa também que, na medida que o produto $m_{0} H_{0}$ diminui (figura 5.3 $(b, c, d))$, os horizontes se afastam um de outro e regiões maiores do espaço-tempo são acessíveis a observadores localizados na região limitada por ambos os horizontes.

Nos gráficos da figura 5.3 se manteve fixo o valor de $H_{0}$ e se modificou o valor de massa $m_{0}$. Se percebe que ambos os horizontes mudam com a mudança de $m_{0}$. Maiores valores de $m_{0}$ 
(a)

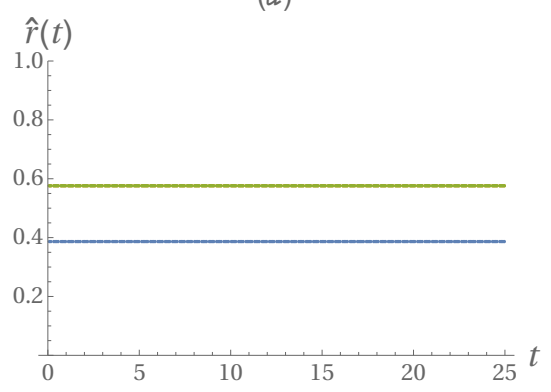

(c)

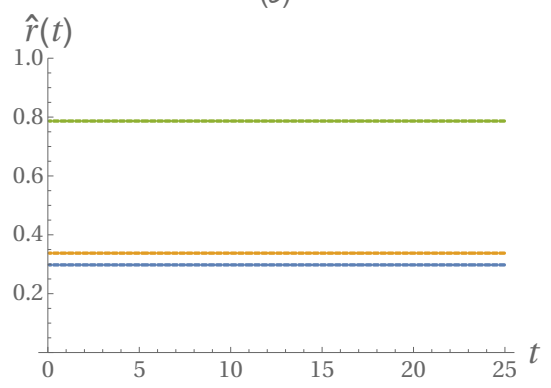

(b)

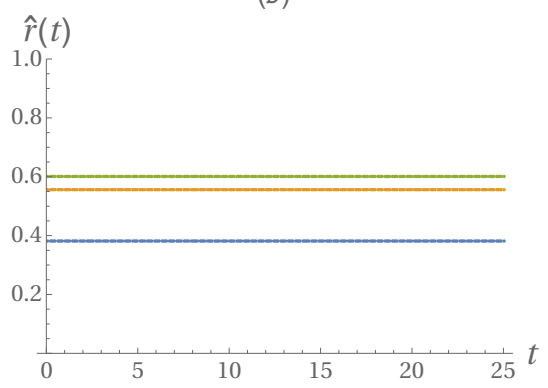

(d)

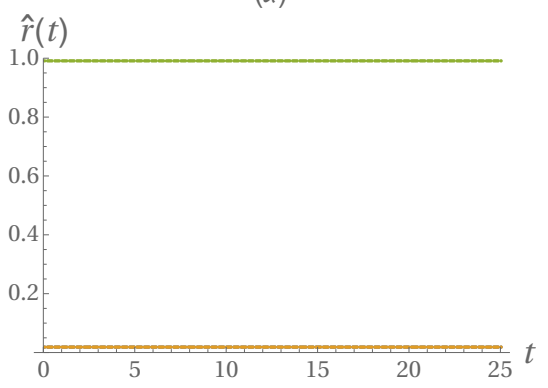

Figura 5.3: Horizontes aparentes da métrica de Schwarzschild-de Sitter em laranja e verde. Em azul se inclui como referência $\hat{r}=2 m_{0}$. Foi usado $H_{0}=1$, e: $(a) m_{0}=0.19245\left(\operatorname{logo}, m_{0} H_{0} \sim 1 / 3 \sqrt{3}\right)$. (b) $m_{0}=0.192(\operatorname{logo}$, $\left.m_{0} H_{0} \lesssim 1 / 3 \sqrt{3}\right)$. (c) $m_{0}=0.15\left(\operatorname{logo}, m_{0} H_{0}<1 / 3 \sqrt{3}\right)$. (d) $m_{0}=0.01\left(\operatorname{logo}, m_{0} H_{0} \ll 1 / 3 \sqrt{3}\right)$.

aproximam o horizonte cosmológico da origem, além de aumentar o tamanho do buraco negro. Na figura 5.4 se observa que a variação do parâmetro $H_{0}$ com $m_{0}$ fixo leva também à mudança de ambos os horizontes. Em termos físicos, quanto maior for a constante cosmológica, maior será a expansão e mais próximo da origem ficará o horizonte cosmológico, tornando maior o buraco negro. Os horizontes aparentes no caso Schwarzschild-de Sitter são representados por linhas retas horizontais em gráficos de $\hat{r}$ em função de $t$, uma característica de um espaço-tempo estático.

\subsection{Casos dinâmicos}

O conjunto de sub-casos que podem ser considerados deste ponto em diante é grande. $\mathrm{Na}$ busca de soluções reais da equação (5.1) a liberdade na escolha das funções $m(t)$ e $H(t)$ está fortemente limitada. Por um lado, tomamos como guia o comportamento das soluções em que a massa do objeto central e o parâmetro de Hubble são constantes (tipo Schwarzschild-de Sitter). Por outro lado, achar comportamentos especiais no nosso polinômio darão condições adicionais que restringem os parâmetros a serem usados.

Um fato importante a ser considerado é que em cada ponto $t_{0}$ em que a equação (5.1) 
(a)

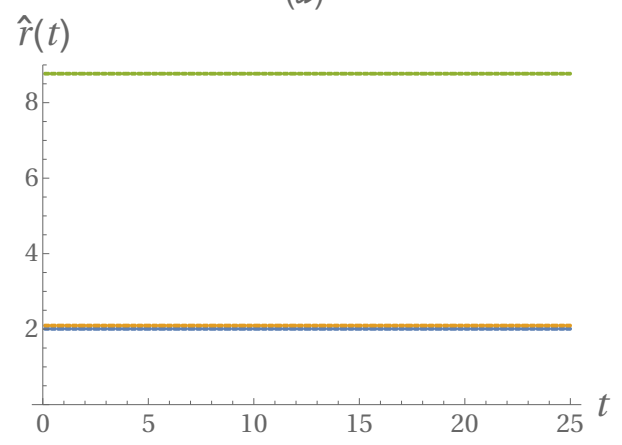

(b)

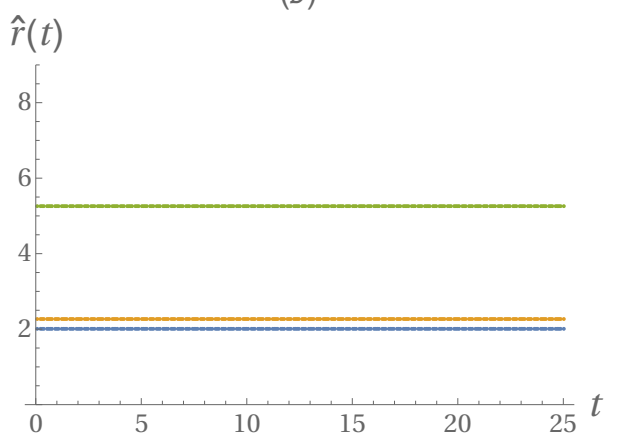

Figura 5.4: Horizontes aparentes da métrica de Schwarzschild-de Sitter em laranja e verde. Foi usado $m_{0}=1$, e: $(a) H_{0}=0.1\left(\operatorname{logo}, m_{0} H_{0}<1 / 3 \sqrt{3}\right)$. (b) $H_{0}=0.15\left(\operatorname{logo}, m_{0} H_{0} \lesssim 1 / 3 \sqrt{3}\right)$. O horizonte cosmológico se aproxima da origem com o aumento de $H_{0}$, enquanto que o horizonte do buraco se afasta dela.

está definida não existe dependência dos coeficientes polinomiais com valores de $t$ fora de um entorno local ao ponto $t_{0}$ (a dependência é no máximo causada por termos com derivadas em $t$ por meio de $M(t)$ ). Logo, soluções em um ponto $t_{0}$ dependem só do que acontece com os coeficientes do polinômio em um entorno de $t_{0}$. Decorre que a condição (5.6) para ter horizontes deve-se satisfazer no caso $s \neq 0, \sigma \neq 0$ ao menos na região em que a massa for constante.

Em regiões em que $M(t)$ for diferente de zero existe a possibilidade de ter uma mudança de sinal no monômio $H(H-2 M) \hat{r}^{4}$ em (5.1). A escolha de parâmetros estará limitada por esse fato como veremos nos análises de gráficos. O caso limite é quando

$$
H=2 M
$$

\subsubsection{Caso $s=0, \sigma \neq 0$ (McVittie)}

Neste caso se adiciona uma variação no parâmetro de Hubble (5.4) considerando em particular as situações $\sigma=1$ e $\sigma=3 / 4$ (correspondentes com as situações $\omega=0$ e $\omega=1 / 3$ na notação usada na seção 4.2.2). Pensando em termos do background ${ }^{1}$ cosmológico FLRW [41], $\sigma=1$ com $H_{0} \neq 0$ representa uma transição entre um universo dominado por matéria em forma de poeira e dominado por constante cosmológica. No entanto que $\sigma=3 / 4$ e $H_{0} \neq 0$ representa uma transição entre um universo dominado por radiação e um dominado por constante cosmológica. Também se tem os casos puros poeira ou radiação com $H_{0}=0$. Existem formas

\footnotetext{
${ }^{1}$ Usamos o termo para denotar a estrutura assintótica do espaço-tempo.
} 


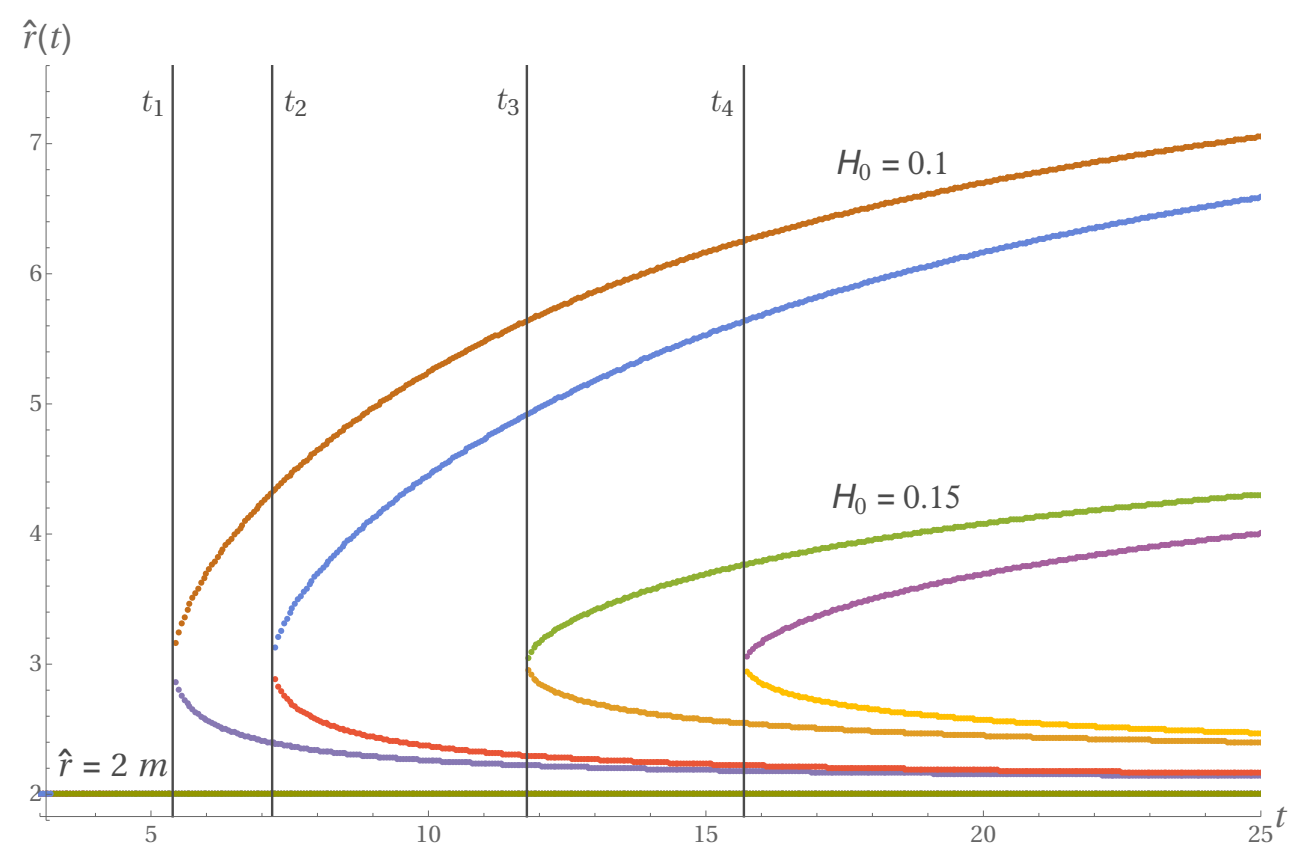

Figura 5.5: Horizontes aparentes nos casos $\sigma=1$ e $\sigma=3 / 4$ para dois valores de $H_{0}$. Ambos os parâmetros definem valores de $t$ para os quais emergem os horizontes. Em marrom e roxo escuro, $H_{0}=0.1, \sigma=3 / 4$. Em azul e vermelho, $H_{0}=0.1, \sigma=1$. Em verde e laranja, $H_{0}=0.15, \sigma=3 / 4$. Em roxo e amarelo, $H_{0}=0.15$, $\sigma=1$. Em verde escuro, $\hat{r}=2 m$ como referência. A diferença entre os casos $\sigma=3 / 4$ e $\sigma=1$ é mínima. Foi usado $m_{0}=1$.

do parâmetro de Hubble mais refinadas (ver por exemplo [12]) que não consideraremos nesse trabalho.

Da expressão (5.4) vemos que a condição (5.6) não será satisfeita em valores arbitrariamente pequenos da coordenada $t$. Podemos escolher os parâmetros $m_{0}$ e $H_{0}$ tais que existe um $t_{0}$ que satisfaz

$$
m_{0} H\left(t_{0}\right)=\frac{1}{3 \sqrt{3}}
$$

e sendo $H(t)$ uma função decrescente, para valores maiores que $t_{0}$ se tem $m_{0} H(t)<1 / 3 \sqrt{3}$ e os horizontes afastarão-se um de outro. Quando $t \rightarrow \infty$ a função $H(t) \rightarrow H_{0}$, e os horizontes se tornam os de Schwarzschild-de Sitter. O polinômio a ser resolvido é dado por

$$
-H^{2} \hat{r}^{4}+2 m_{0} H^{2} \hat{r}^{3}+\hat{r}^{2}-4 m_{0} \hat{r}+4 m_{0}^{2}=0
$$

que de novo se reduz a um polinômio de terceiro grau na região em que o $\hat{r}$ está definido. A figura 5.5 mostra os horizontes quando $\sigma=1$ e $\sigma=3 / 4$ para dois valores de $H_{0}$. O surgimento dos horizontes na coordenada $t$ depende de ambos os parâmetros. As linhas negras verticais marcam os valores em $t$ para os quais surgem os horizontes nos diferentes casos. $\mathrm{O}$ valor da 
massa $m_{0}$ foi fixado, as curvas maiores representam um mesmo valor de $H_{0}$ da mesma forma que as curvas menores. Ressaltamos que a diferença nas curvas para um mesmo valor de $H_{0}$ e $m_{0}$ não é importante para o análise qualitativo feito neste trabalho.

Para $t<t_{0}$ não existem horizontes aparentes, temos apenas uma singularidade nua em $\hat{r}=$ $2 m_{0}$ [37] onde o escalar de Ricci e a pressão divergem. Em $t=t_{0}$ aparecem simultaneamente os horizontes aparentes no mesmo valor de $\hat{r}$ e com o aumento de $t$ eles se bifurcam. O horizonte de buraco negro aproxima-se assintoticamente da singularidade $\hat{r}=2 m_{0}$.

Quanto maior for a constante $H_{0}$ escolhida mais afastado da origem em $\hat{r}$ estará o horizonte do buraco e mais próximo o cosmológico. O limite estará dado pela violação da condição (5.6). Quanto menor for o $H_{0}$ mais próximo estará o horizonte do buraco da origem tendo como limite o caso em que $H_{0}=0$ (estamos considerando $H_{0} \geqslant 0$ ). Substituindo a expressão da família de parâmetros de Hubble (5.4) na condição que define a $t_{0}$ (5.8), é possível mostrar que a seguinte expressão dá o valor de $t_{0}$ para o qual emergem os horizontes em termos da massa inicial do objeto, da constante cosmológica e do valor de $\sigma$

$$
t_{0}=\frac{2 \sqrt{3} m_{0} \sigma}{1-3 \sqrt{3} m_{0} H_{0}},
$$

em que se observa que o valor da massa $m_{0}$ tem um peso grande no numerador da fração. $\mathrm{O}$ produto $m_{0} H_{0}$ tem que ser no máximo $1 / 3 \sqrt{3}$ se quisermos garantir a existência dos horizontes. A expressão é válida quando o parâmetro de Hubble tem a forma dada em (5.4) e quando $m_{0}$ seja tal que o correspondente $M_{0}$ for nulo no ponto em que $m_{0}$ é definido. No caso limite em que a constante cosmológica for zero, o valor mínimo em $t$ para o qual os horizontes emergem será nesse caso

$$
t_{\text {min }}=2 \sqrt{3} m_{0} \sigma
$$

Na figura 5.6 se observa o caso limite dos horizontes correspondente com um fundo cosmológico dominado por matéria ou radiação $(\sigma=1$ ou $\sigma=3 / 4)$. Conforme tem lugar a expansão, o horizonte cosmológico se afasta cada vez mais da origem. Mais rápido no caso do universo dominado por radiação. Por outro lado os horizontes tipo buraco negro se aproximam ao limite $\hat{r}=2 m=2 m_{0}$. 


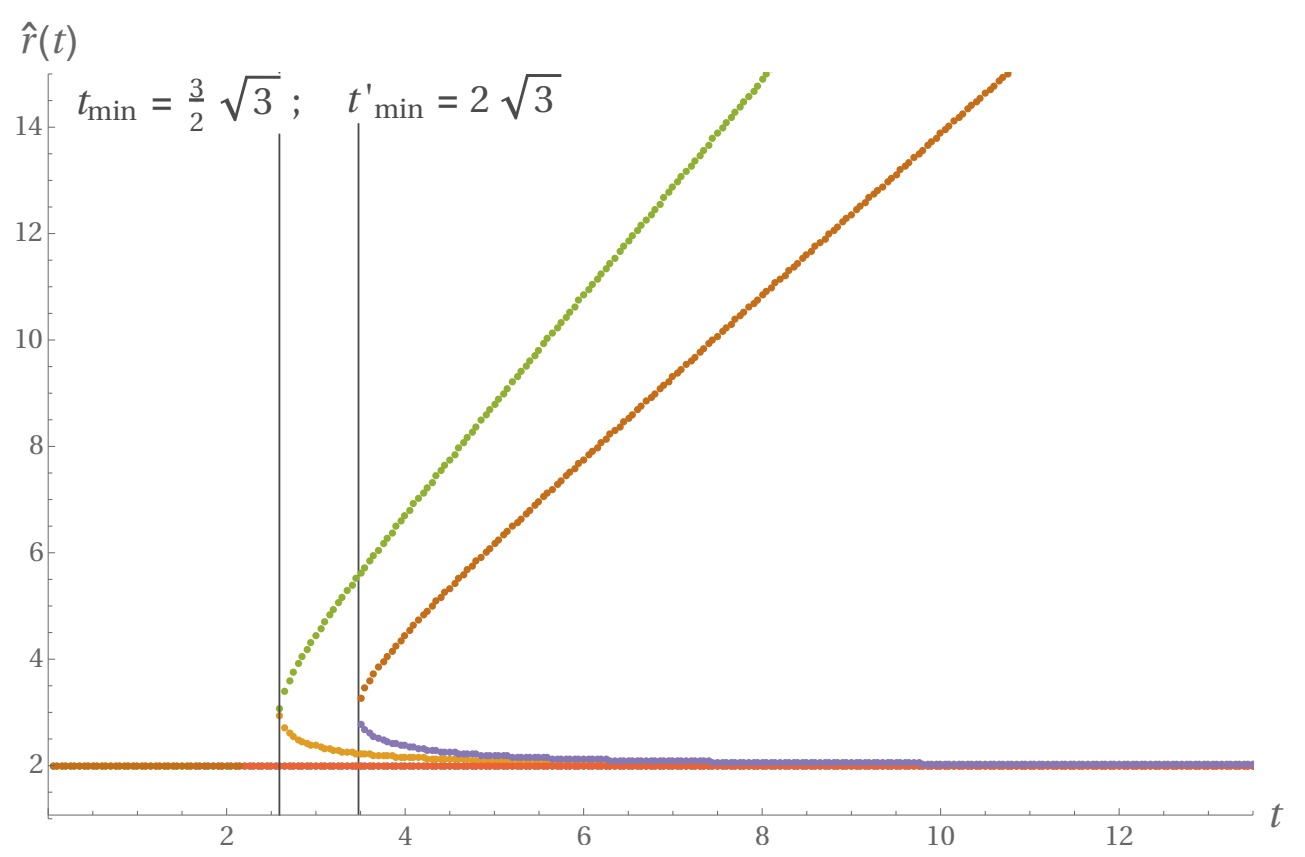

Figura 5.6: Horizontes aparentes no caso $\sigma=1$ e $\sigma=3 / 4$ com $H_{0}=0$. Como referência, $\hat{r}=2 m$ está incluído. Foi usado $m_{0}=1$.

\subsubsection{Caso $s \neq 0, \sigma=0$ (McVittie generalizado em fundo de Sitter)}

Tendo acreção de massa no objeto central $(s \neq 0)$, consideramos primeiro o caso que segundo o modelo cosmológico $\Lambda$-CDM corresponde com uma etapa de expansão acelerada do universo [41] desde que $H_{0}$ seja maior que zero.

Pode-se interpretar o sistema físico considerado como sendo um objeto esférico submerso em um fluido imperfeito, em expansão acelerada e acretando massa. Um caso especial de buraco negro cosmológico. Consideramos um bom cenário no qual buracos negros primordiais poderiam ter surgido. Sendo o parâmetro $s$ que controla o gap na massa inicial. O polinômio neste caso é

$$
H_{0}\left(H_{0}-2 M\right) \hat{r}^{4}-2\left(m\left(H_{0}-M\right)^{2}-M\right) \hat{r}^{3}-(4 m M+1) \hat{r}^{2}+4 m \hat{r}-4 m^{2}=0 .
$$

Soluções em um ponto $t_{0} \in\left(0, t_{1}\right) \cup\left(t_{2}, \infty\right)$ são independentes da acreção em $t_{1}<t<t_{2}$ graças ao argumento da seção anterior. No intervalo $\left(0, t_{1}\right) \cup\left(t_{2}, \infty\right)$ em que a massa é constante pode-se esperar que as soluções sejam as mesmas obtidas no caso Schwarzschild-de Sitter.

A condição (5.6) será usada como guia principal na escolha de parâmetros na busca de soluções de (5.12). A tripla particular $\left\{m_{0}, H_{0}, s\right\}=\{1.0,0.1,0.4\}$ define um estado inicial do sistema em que $m_{0} H_{0}=0.1<1 / 3 \sqrt{3}$ e um estado final em que (usando a definição da função 
massa (5.2)) $\left(m_{0}+2 s\right) H_{0}=0.18<1 / 3 \sqrt{3}$. Ou seja, onde existem dois horizontes aparentes diferentes ao menos na região de massa constante.

A figura 5.7 mostra o comportamento das soluções de (5.12) sob mudanças no valor do gap $s$. Observa-se que maiores valores do gap $s$ correspondem com estados finais em que os horizontes ficam mais próximos sendo o buraco negro maior que no início. Na região de acreção os horizontes se curvam um para o outro na medida que o gap for maior. Chegando no ponto em que os horizontes se juntam e dividem o espaço-tempo numa forma peculiar em que qualquer curva dirigida ao futuro acaba inevitavelmente travessando algum dos horizontes os quais emergem novamente em tempos posteriores.

(a)

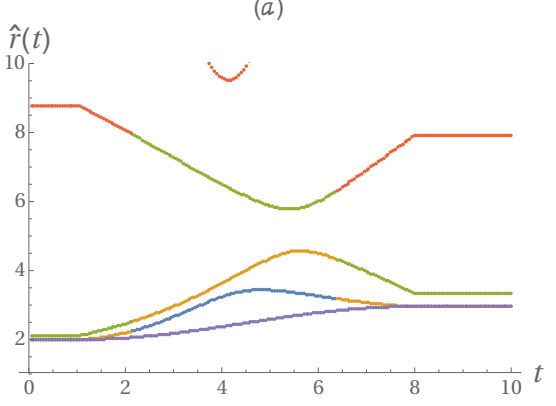

(c)

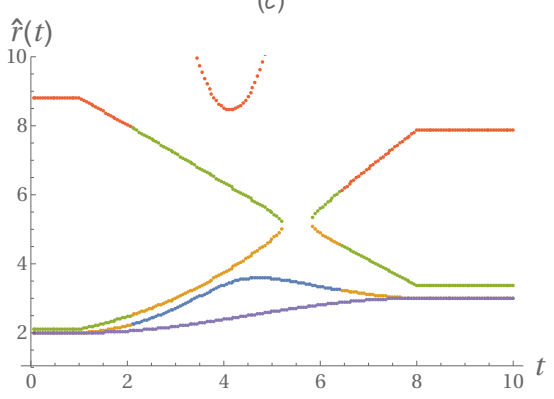

(b)

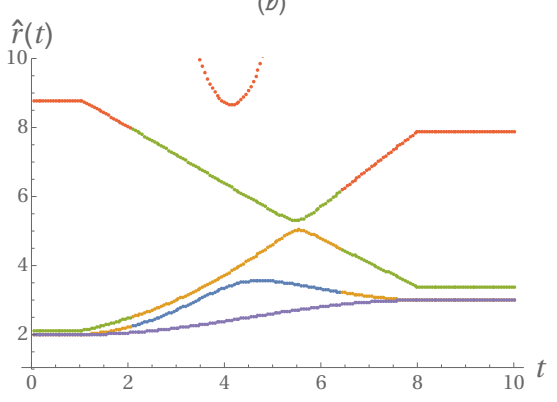

(d)

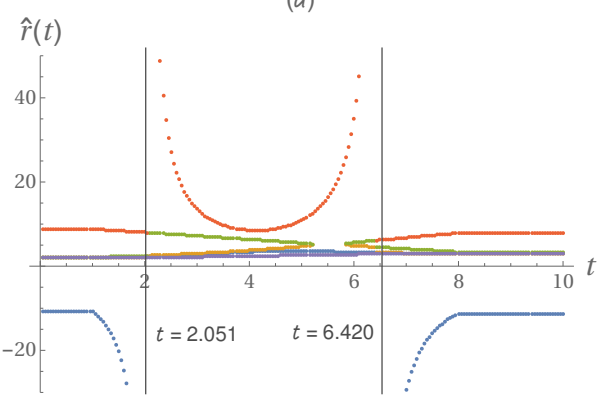

Figura 5.7: Horizontes aparentes inicialmente bem comportados mas com o incremento do gap terminam se juntando e criando um estrutura mais complexa. Em roxo, $\hat{r}=2 m$ como referencia. Foi usado $m_{0}=1, t_{1}=1, t_{2}=$ $8, H_{0}=0.1$. Também $(a) s=0.24,(b) s=0.248,(c)$ e $(d) s=0.25$.

Se observa também uma curva solução que coincide com $\hat{r}=2 m$ quando a massa é constante a qual corresponde com a singularidade. Uma outra curva é só visualizada quando se aumentar o range do plot a valores negativos de $\hat{r}$, figura 5.7(d). Resulta interessante considerála porque é parte da outra curva superior em laranja que se aproxima com o incremento do gap até ela travessar os horizontes como se mostra na figura $(5.8)(a, b, c)$. 
$(a)$

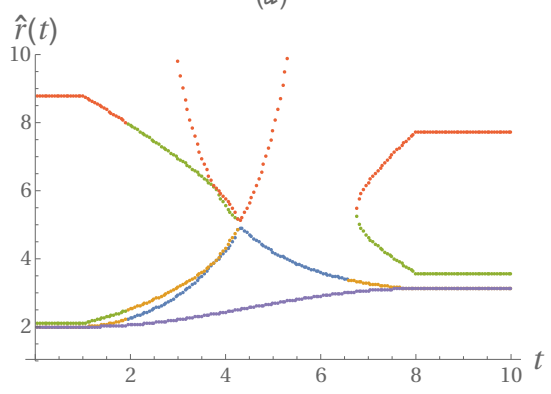

(c)

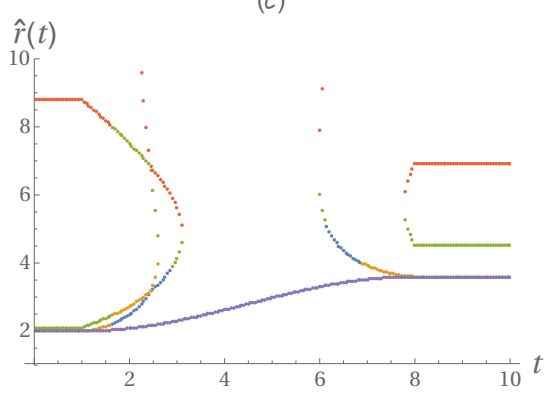

(b)

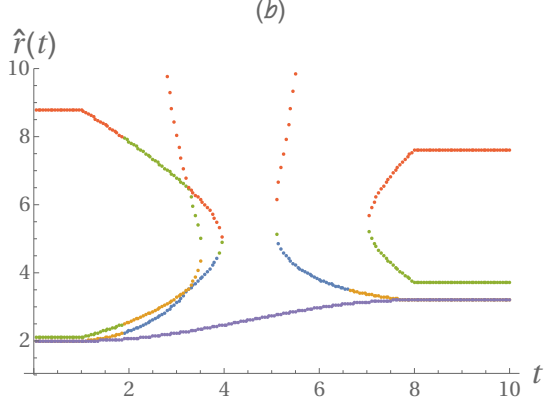

(d)

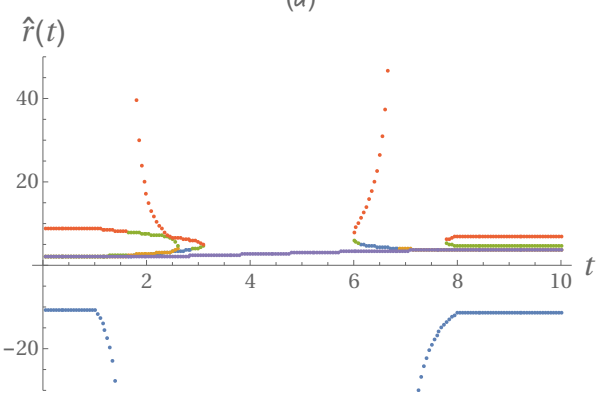

Figura 5.8: Vários valores de $s$ mostram como pode-se complicar a estrutura de horizontes no espaço-tempo. Em roxo, $\hat{r}=2 m$. Foi usado $m_{0}=1, t_{1}=1, t_{2}=8, H_{0}=0.1$. Também $(a) s=0.28,(b) s=0.3,(c)$ e $(d)$ $s=0.4$.

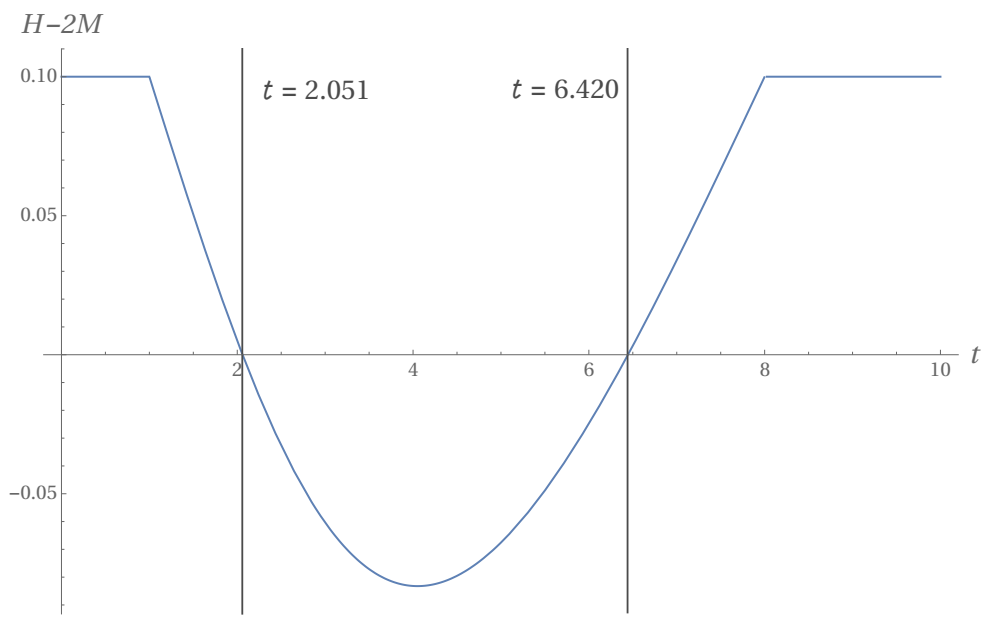

Figura 5.9: Os valores em que a curva é nula são os mesmos em que as descontinuidades em 5.7(d) aparecem. Foi usado $m_{0}=1, t_{1}=1, t_{2}=8, H_{0}=0.1, s=0.25$.

Porém, uma escolha adequada de parâmetros evita tais comportamentos. A presença da curva superior em laranja nas figuras 5.7-5.8 está associada com a condição (5.7). Correspondente com os parâmetros usados no gráfico $5.7(d)$, na figura 5.9 se vê a coincidência nos valores de $t$ em que aparecem as descontinuidades com os valores em que se satisfaz $H=2 M$.

Levando em conta a restrição imposta por (5.7) se consegue uma escolha dos parâmetros em que os horizontes solução de (5.12) não apresentam comportamentos atípicos, conforme 


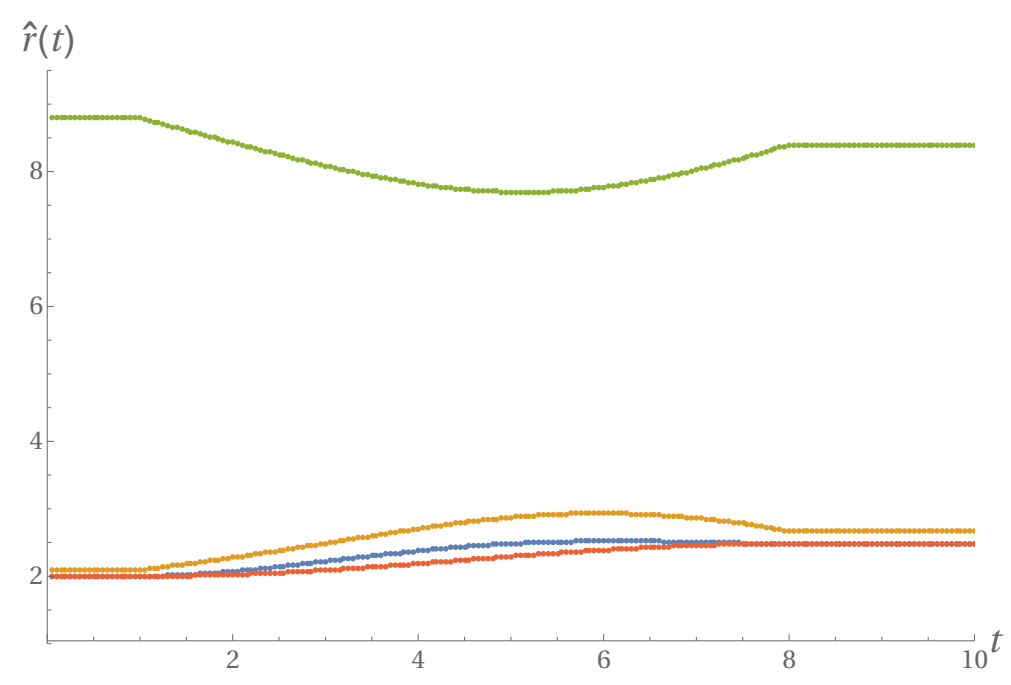

Figura 5.10: As descontinuidades e os comportamentos estranhos são evitados com a escolha certa de parâmetros. Em verde o horizonte cosmológico, em laranja o horizonte do buraco negro, em azul a solução associada à singularidade, em vermelho $\hat{r}=2 m$ como referência. Foi usado $m_{0}=1, t_{1}=1, t_{2}=8, H_{0}=0.1, s=0.12$.

ilustrado na figura 5.10.

\subsubsection{Caso $s \neq 0, \sigma \neq 0$ (McVittie generalizado em fundo FLRW)}

Passamos agora a estudar algumas situações mais complexas. Consideramos um background de tipo FLRW (em particular $\sigma=1$ por simplicidade e em vista de que não existem maiores diferenças com o caso $\sigma=3 / 4$ ) em um estado de transição entre um universo dominado por matéria e dominado por energia escura. Consideramos um bom lugar para estudar buracos negros cosmológicos e pensá-los como possíveis buracos negros primordiais. Existe flexibilidade a comparação com o caso anterior em que só havia constante cosmológica e o background era de Sitter. O polinômio a ser considerado é (5.1) na sua forma original com funções dependentes do tempo.

A figura 5.11 mostra como é a formação dos horizontes para vários dos valores de $H_{0}$. Linhas negras verticais longas limitam a região na abscisa na qual ocorre acreção de massa do objeto central. As setas indicam valores de $t$ para os quais é previsível com (5.10) o surgimento dos horizontes. A criação dos horizontes pode o não estar dentro do intervalo de acreção. Quando estiver fora, a condição (5.8) é satisfeita. Adicionalmente se mostra a curva $\hat{r}=2 \mathrm{~m}$ como referência.

Se observa que no processo de acreção de massa os horizontes se curvam um para o outro aproximando-se mais do que finalmente estarão. Uma combinação adequada de parâmetros 


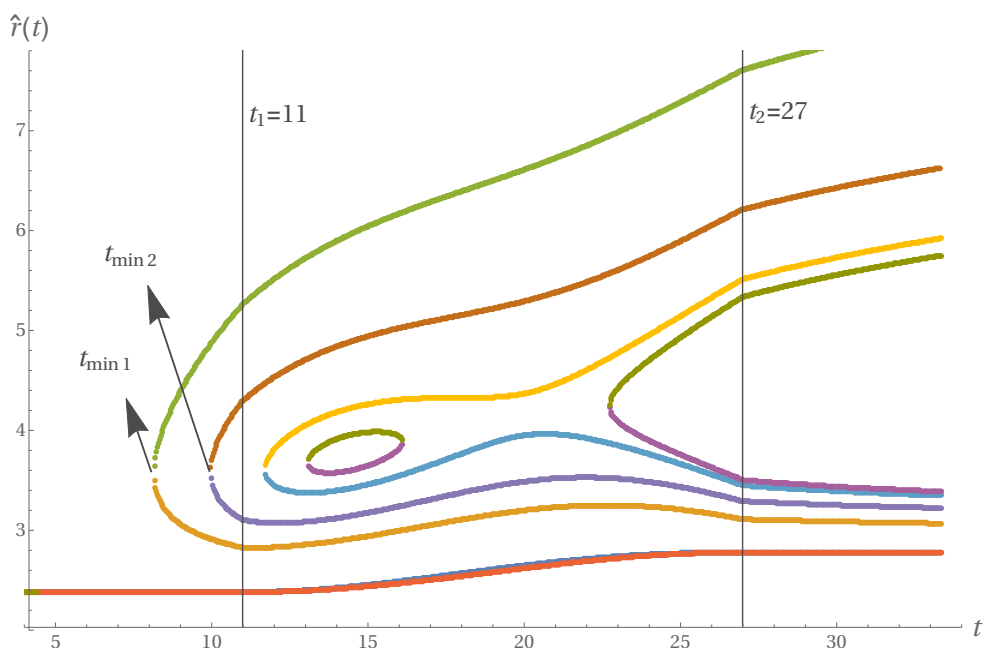

Figura 5.11: Horizontes aparentes no caso dinâmico $\sigma=1$ com acreção de massa e vários valores de $H_{0}$. Foi usado $m_{0}=1.19, t_{1}=11, t_{2}=27, s=0.1$, em verde e laranja $H_{0}=0.08$, em marrom e roxo escuro $H_{0}=0.095$, em amarelo e azul $H_{0}=0.103$, em verde escuro e roxo $H_{0}=0.105$. Em vermelho $\hat{r}=2 m$ como referência.

pode gerar momentaneamente a existência do par de horizontes até que um colapsa com o outro nesse processo de aproximação entre eles. Existe a tentativa dos horizontes manter sua existência mas não conseguem por causa de ter um mecanismo de acreção muito violento nas condições colocadas. Mas podendo surgir de novo quando a acreção a comparação com a expansão for fraca o suficiente para compensar, conforme as curvas plotadas em verde e laranja na figura 5.11. Para apreciar melhor os detalhes dos horizontes de buraco foi plotado o gráfico da figura 5.12.

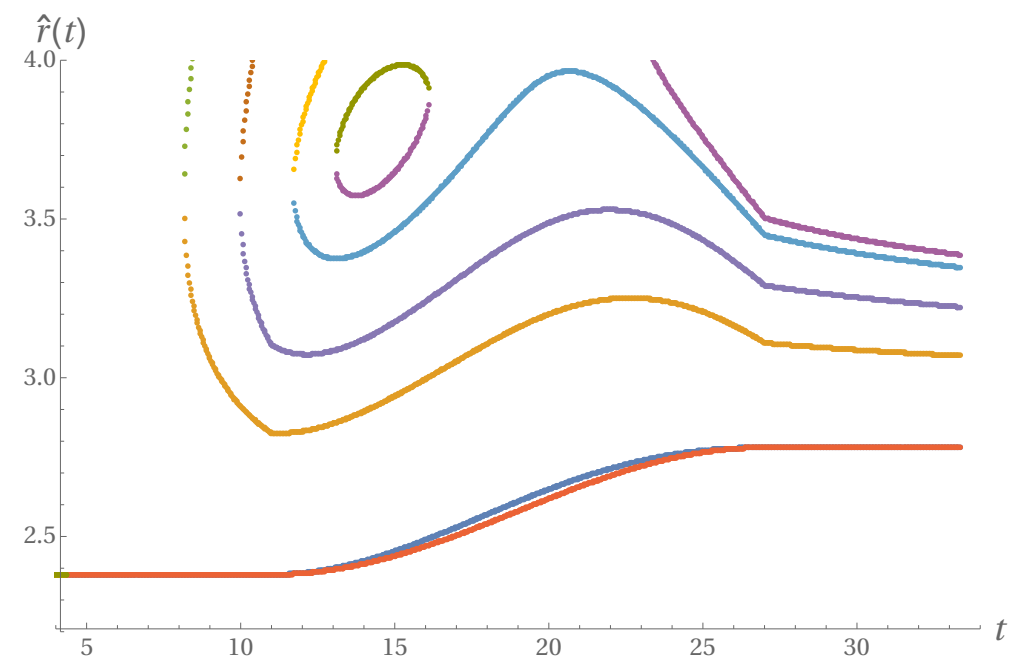

Figura 5.12: Horizontes aparentes do buraco negro da figura 5.11 em detalhe, onde se observa a mudança no tamanho de buraco negro com o aumento da massa. Em vermelho $\hat{r}=2 m$, como referência.

Porém, essa interpretação implicaria que existe acreção em momentos em que não exis- 


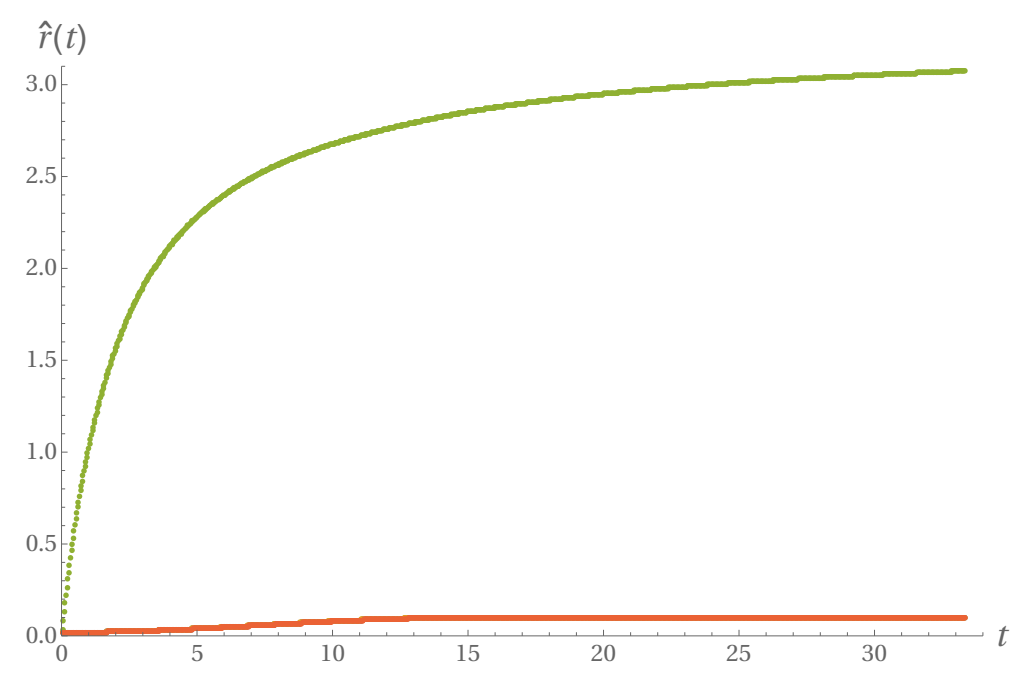

Figura 5.13: Surgimento dos horizontes aparentes a valores de $t$ pequenos. Se considera uma inomogeneidade inicial bastante pequena. Foi usado $m_{0}=0.0093868, t_{1}=0.033, t_{2}=15, s=0.02, H_{0}=0.3$. A acreção considerada não é grande o suficiente para afastar o horizonte aparente da curva $\hat{r}=2 \mathrm{~m}$.

tem os horizontes, o que seria uma possibilidade se existir geodésicas tipo tempo que acabam na singularidade nesses instantes em que não existem horizontes. Deixamos em aberto essa questão.

Por outro lado, pode ser interessante considerar acreção apenas a partir do momento em que os horizontes emergem. Estaríamos considerando uma partícula de massa inicial $m_{0}$ que começa a ganhar massa do fluido no qual está submersa no momento em que os horizontes começaram a existir, o que pode ser interpretado como um buraco negro primordial. Junto com isto, podemos considerar a massa inicial como sendo a mínima possível compatível com o surgimento dos horizontes em $t>0$.

Consideramos a expressão (5.10) para construir a solução quando a inomogeneidade for bastante pequena e o surgimento dos horizontes ficarem perto da origem no $t$ (valores enormes do parâmetro de Hubble). A figura 5.13 mostra essa situação em que se observa que o horizonte de tipo buraco acompanha o comportamento de $\hat{r}=2 m$ devido ao mecanismo de acreção que não é forte o suficiente para afastar o horizonte novo do caso do McVittie original [20]. Se espera que com o aumento de valores de tempo $t$ os horizontes se aproximem aos horizontes do caso Schwarzschild-de Sitter chegando num ponto estável da existência do buraco negro em um background dominado pela constante cosmológica. A forma da curva na figura 5.13 se mantém apenas com um limitada escolha de parâmetros. Pequenas mudanças fazem com que apareçam descontinuidades, devido a condição (5.7). 



\section{Capítulo 6}

\section{Conclusões}

Neste trabalho se estudaram espaço-tempos dinâmicos de tipo buraco negro em um ambiente cosmológico no contexto da teoria da relatividade geral de Einstein. Em particular, considerouse a generalização de massa variável do espaço-tempo de McVittie. Foram apresentados os espaço-tempos limites da solução de McVittie generalizada os quais incluem Schwarzschild, FLRW, Schwarzschild-de Sitter e McVittie. Discutimos a definição padrão de buraco negro e horizonte de eventos assim como as dificuldades de aplicá-las em situações dinâmicas. Outras definições para horizontes foram exploradas e usamos o conceito de horizonte aparente para localizar buracos negros e horizontes cosmológicos. Foram encontradas soluções para o problema da existência dos horizontes aparentes nas geometrias de Schwarzschild-de Sitter, McVittie e McVittie generalizado.

Um ponto relevante do desenvolvimento feito deve ser ressaltado. Os horizontes aparentes de buraco negro e cosmológico encontrados no capítulo 5 partem da solução da mesma equação $\tilde{H} \hat{r}-R=0$, que corresponde com a anulação de $\theta_{\boldsymbol{n}}$ (expansão de geodésicas ingoing) no sistema de coordenadas em que a métrica de McVittie generalizada se expressa na forma (4.37). No entanto que $\theta_{\ell}$ não se anula (4.70), sempre é positivo na região de interesse $(\hat{r}>2 m)$. Por outro lado, ambas as expansões são nulas nas coordenadas de Schwarzschild levando à equação $f(t, \hat{r})=0$ equivalente a (4.71). A definição de horizontes aparentes segundo Faraoni [37] precisa que $\theta_{\ell}=0, \theta_{n}<0$ para ser um horizonte aparente futuro (horizonte tipo buraco negro) e $\theta_{n}=0, \theta_{\ell}>0$ para ser um horizonte aparente passado (horizonte cosmológico). Foi usado a definição como guia e no caso da métrica de McVittie generalizada, as soluções obtidas foram identificadas com os horizontes de buraco negro e cosmológico porque nos casos limite 
correspondem com horizontes desses tipos em uma métrica bem conhecida (Schwarzschildde Sitter) mas não por satisfazer as condições que definem os horizontes aparentes segundo o Faraoni.

Para esclarecer a questão, está sendo explorada a possibilidade de ter outras regiões da geometria inacessíveis nas coordenadas usadas neste trabalho. Também poderiam ser consideradas outras definições para horizontes. Por exemplo o formalismo de Hayward [38] precisa que a foliação do espaço-tempo seja em coordenadas nulas e além de pedir anulação da expansão de uma das congruências e não nulidade da outra das congruências, impõe condições sobre as derivadas das expansões. A formulação é muito mais do que aqui ilustramos como resumo. Com isto, se classificam os trapping horizons em past ou future e inner ou outer. As geometrias generalizadas de McVittie podem ser estudadas usando o formalismo de Hayward, embora algumas dificuldades possam ser previstas antecipadamente como é o caso da não linearidade na transformação de coordenadas nulas relacionadas ao cálculo das expansões e as suas derivadas.

A família de parâmetros de Hubble escolhida modelando backgrounds cosmológicos impõe um valor mínimo no tempo para o qual os horizontes emergem nos casos de tipo McVittie para determinado valor de massa $m_{0}$ da partícula considerada. O cálculo foi feito com a condição que a formação dos horizontes deve estar fora do intervalo em que a massa é variável, ou seja, onde a existência dos horizontes aparentes está determinada por um polinômio de terceiro grau com condição para a existência de soluções reais bem conhecidas. Valores menores no parâmetro de massa permitem a formação cada vez mais antecipada dos horizontes. A ideia de existir acreção antes dos horizontes se formarem, como um mecanismo que ajudaria a explicar a formação dos mesmos não pode ser confirmada ou descartada com a nossa análise. A resposta daquela questão está associada com a forma das geodésicas tipo tempo, ou pela região tipo tempo limitada por geodésicas tipo luz que podem existir para $t<t_{0}$. Em [20] são apresentadas soluções de geodésicas ingoing formadas antes dos horizontes. Estas curvas nunca atingem a singularidade, mas sobrepassam o horizonte do buraco negro. Isso indica que existe a possibilidade de acrescentar a massa do buraco negro com eventos que acontecem antes da formação dos horizontes. Não sugerimos uma resposta definitiva para esta questão, e o trabalho nesta linha de pesquisa está em andamento.

Quando se consideram os casos dinâmicos, o tamanho dos horizontes muda em formas diferentes em cada caso. No caso McVittie, a área do horizonte do buraco negro sempre diminui e o horizonte cosmológico sempre aumenta. No caso McVittie generalizado com background 
de Sitter, os horizontes mudam na forma esperada entre os estados estacionários ( $m$ constante) inicial e final (figura 5.10). A área do buraco negro aumenta logo depois do processo dinâmico de acreção. Porém, o tamanho dos horizontes atinge um valor extremo durante a acreção que supera as vezes o valor final. A derivada da área do buraco negro em relação ao tempo $d A / d t=$ $8 \pi \hat{r} d \hat{r} / d t$ pode ser negativa em um conjunto contido no intervalo $\left(t_{1}, t_{2}\right)$, como é o caso no McVittie generalizado em background FLRW (figuras 5.11 ou 5.12). Por outro lado, com uma escolha de parâmetros adequada o processo de acreção pode acontecer devagar o suficiente para ter derivada da área do buraco negro sempre positiva como mostrado na figura 5.13. Será interessante estudar as implicações termodinâmicas disto.

A flexibilidade que existe em mudar os parâmetros de forma de localizar o surgimento dos horizontes em alguma época na evolução do fator de escala e inclusive de considerar outras formas de acreção por meio da função massa $m(t)$, dá a possibilidade de usar modelos de McVittie generalizados para descrever buracos negros primordiais. Se tem por exemplo [46] onde se propõe uma forma da função de massa no processo de evaporação dos buracos negros. Investigaremos como tais modelos se ajustam no contexto cosmológico atual. 



\section{Apêndice A}

\section{Variedades diferenciais}

No que segue fazemos um resumo com um enfoque não tão rigoroso das ideias matemáticas que constituem a base do que são as variedades diferenciais. Para maiores detalhes, a referência guia na elaboração desse material foi [47].

Seja $X$ um conjunto qualquer e $\mathcal{P}(X)$ o conjunto de todos os subconjuntos de $X$. Uma topologia $\tau$ em $X$ é uma coleção de subconjuntos em $\mathcal{P}(X)$ que satisfaz as seguintes propriedades:

1. $\emptyset \in \tau, X \in \tau$;

2. se $A, B \in \tau \Rightarrow A \cap B \in \tau$;

3. se $A_{\lambda} \in \tau, \lambda \in \Lambda \Rightarrow \bigcup_{\lambda \in \Lambda} A_{\lambda} \in \tau$.

Um espaço topológico é um conjunto $X$ dotado de una topologia $\tau$ e denotado como $(X, \tau)$. Se diz que um conjunto $A \in \tau$ é $\tau$-aberto. A topologia $\tau$ está constituída por uma coleção de conjuntos definidos como abertos. O complemento dessa coleção como fechado. Um conjunto $F \subset X$ se diz $\tau$-fechado se o seu complemento $F^{c}=X \backslash F$ for $\tau$-aberto.

Uma topologia dá uma noção de continuidade de funccões que mapeiam espaços topológicos. Sejam $\left(X, \tau_{X}\right)$ e $\left(Y, \tau_{Y}\right)$ espaços topológicos, e seja uma função $f: X \rightarrow Y$. Seja o conjunto $B \in \tau_{Y}$ a imagem de algum conjunto em $X$ que chamamos pré-imagem de $B$ por meio de $f$ (ver figura A.1), ou seja, ao conjunto

$$
f^{-1}(B)=\{x \in X \mid f(x) \in B\} .
$$


Se diz que $f$ é $\left(\tau_{X}, \tau_{Y}\right)$-contínua se $f^{-1}(B) \in \tau_{X} \forall B \in \tau_{Y}$. Se existir $f^{-1}: Y \rightarrow X$. Se

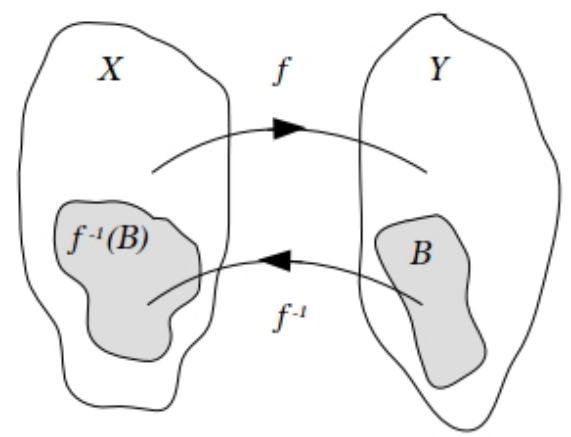

Figura A.1: A função $f$ mapeia elementos de $X$ em $Y$. Se representa por $f^{-1}$ ao mapa que leva elementos de $Y$ a suas pre-imágens en $X$.

diz que $f$ é um homeomorfismo de $\left(X, \tau_{X}\right)$ em $\left(Y, \tau_{Y}\right)$ se for $\left(\tau_{X}, \tau_{Y}\right)$-contÃnua e $f^{-1}$ for $\left(\tau_{Y}, \tau_{X}\right)$-contínua.

Para introduzir posteriormente o conceito de variedade topológica, vai ser importante introduzir o conceito de espaço Hausdorff. Um espaço topológico $(X, \tau)$ é dito ser Hausdorff se existir abertos $A_{x}, A_{y} \in \tau$ para todos $x, y \in X \operatorname{com} x \neq y$ tais que $x \in A_{x}, y \in A_{y}$ e $A_{x} \cap A_{y}=\emptyset$. Um resultado a propósito disso é que todo espaço métrico é Hausdorff.

Seja um conjunto $X$ e sejam $\tau_{1}, \tau_{2} \in \mathcal{P}(X)$ duas possíveis topologias em $X . \tau_{1} \cap \tau_{2}$ também é uma topologia em $X$. Em geral uma coleção de topologias $\mathcal{T}=\left\{\tau_{\mu}, \mu \in \Omega\right\}$ (sendo $\Omega$ algum conjunto indexado) forma de novo uma topologia dada pela coleção $\tau_{I}=\bigcap_{\mu \in \Omega} \tau_{\mu}$.

Sejam os conjuntos $X$ e $A \subset X$. Sempre existem as seguintes topologias:

$$
\begin{aligned}
\tau & =\mathcal{P}(X), \\
\tau_{A} & =\{B \subset X \mid A \subset B\} \cup\{\emptyset\} .
\end{aligned}
$$

Chamemos de $\mathcal{T}_{A}$ à coleção de topologias que contém o conjunto $A, \mathcal{T}_{A}=\left\{\tau_{\mu}, \mu \in \Omega \mid\right.$ $\tau_{\mu}$ é uma topologia, $\left.A \in \tau_{\mu}\right\}$ (com $\Omega$ um conjunto indexado). A topologia gerada pelo conjunto $A$ denotada $\tau[A]$ sendo a menor das topologias que contém o conjunto $A$ se define como

$$
\tau[A]=\bigcap_{\tau_{\mu} \in \mathcal{T}_{A}} \tau_{\mu}
$$

Sendo $\mathcal{A} \subset P(X)$ uma coleção de subconjuntos de $X$. A coleção de topologias que contém a 
coleção $\mathcal{A}, \mathcal{T}_{\mathcal{A}}=\left\{\tau_{\mu}, \mu \in \Omega \mid \tau_{\mu}\right.$ é uma topologia, $\left.\mathcal{A} \subset \tau_{\mu}\right\}$. Então a topologia gerada pela coleção $\mathcal{A}$ está definida como sendo

$$
\tau[\mathcal{A}]=\bigcap_{\tau_{\mu} \in \mathcal{T}_{\mathcal{A}}} \tau_{\mu}
$$

Seja um espaço topológico $(X, \tau)$. A coleção $\mathcal{A}$ é dita ser uma sub-base de $\tau$ se $\tau=\tau[\mathcal{A}]$, ou seja, se for a menor topologia que contém a coleção $\mathcal{A}$.

Em um espaço topologico $(X, \tau)$, uma coleção $\mathcal{B} \subset \tau$ é dita ser uma base de $\tau$ se para todo $A \in \tau$ existir $\left\{B_{\lambda} \in \mathcal{B}, \lambda \in \Lambda\right\}$ ( $\Lambda$ um conjunto indexado) tal que $A=\bigcup_{\lambda \in \Lambda} B_{\lambda}$. Toda base é também uma sub-base. Um espaço topológico $(X, \tau)$ é dito ser segundo-contável se possuir uma base contável.

Em $\mathbb{R}^{n}$ com métrica usual $\|x-y\|=\sqrt{\left(x^{1}-y^{1}\right)^{2}+\ldots+\left(x^{n}-y^{n}\right)^{2}}$ uma bola aberta centrada em $x \in \mathbb{R}^{n}$ de radio $r>0$ está formada pelo conjunto

$$
D_{n}(r, x)=\left\{y \in \mathbb{R}^{n} \mid\|x-y\|<r\right\} .
$$

Todas as bolas abertas em $\mathbb{R}^{n}$ (com $n$ fixo) $D_{n}(r, x)$ são homeomorfas entre si. Ou seja, existe uma $f: D_{n}(r, x) \rightarrow D_{n}\left(r^{\prime}, x^{\prime}\right)$ bijetora, contínua e com inversa contínua.

Agora seja um espaço topológico $(X, \tau)$ e seja $V$ um aberto $V \subset X$. Se diz que $V$ é um aberto euclidiano de dimensão $n$ se for homeomorfo a $D_{n}\left(r_{v}, 0\right)$ para algúm $r_{v}>0$. Um comentário importante é que a bola centrada em cero é homeomorfa a qualquer bola em $\mathbb{R}^{n}$. Logo, existe a correspondência entre um aberto de $X$ e uma bola centrada em cero em $\mathbb{R}^{n}$ (figura A.2). A correspondência é unÃvoca.

Dado um espaço topológico $(X, \tau)$, denotaremos por $\varepsilon(X, \tau, n)$ a coleção de todos os abertos euclidianos de $(X, \tau)$ que é de novo um aberto $(\varepsilon(X, \tau, n) \subset \tau)$. Se diz que $(X, \tau)$ é localmente euclidiano de dimensão $n$ se $\varepsilon(X, \tau, n)$ for um recobrimento de $X$, ou seja,

$$
X \subset \bigcup_{V \in \varepsilon} V
$$

Uma variedade topológica segundo-contável é um espaço topológico $(X, \tau)$ Hausdorff, localmente euclidiano e segundo-contável.

Seja $(X, \tau)$ localmente euclidiano de dimensão $n$. A dupla $(V, h), \operatorname{com} V \in \varepsilon(X, \tau, n)$ um 


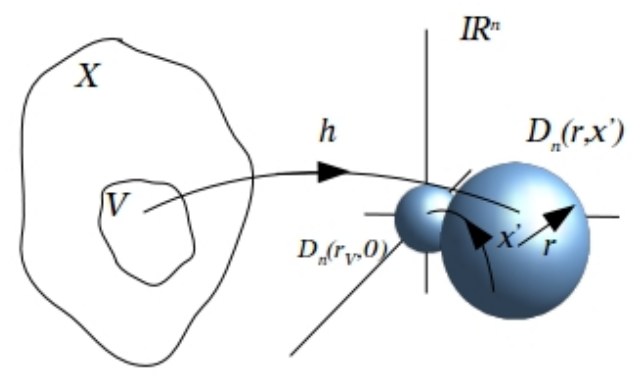

Figura A.2: Um aberto $V \subset X$ homeomorfo a uma bola aberta em $\mathbb{R}^{n}$ por sua vez essa bola é homeomorfa com uma centrada em cero. $V$ é um aberto euclidiano de dimensão $n$.

aberto euclidiano de $X$ e $h$ um homeomorfismo de $V$ para algum $D_{n}(r, 0) \subset \mathbb{R}^{n}$ é chamado carta local de coordenadas do aberto $V$. Uma coleção $\mathcal{A}=\left\{\left(V_{\lambda}, h_{\lambda}\right), \lambda \in \Lambda\right\}$ (com $\Lambda$ um conjunto indexado) é dito ser um ( $n$-atlas) em $(X, \tau)$ se $\left(V_{\lambda}, h_{\lambda}\right)$ foram cartas locais de coordenadas e constituam um recobrimento para $X$.

Seja $(X, \tau)$ um espaço topológico e sejam os pares $\left(U, h_{U}\right)$ y $\left(V, h_{V}\right)$ duas cartas locais de coordenadas onde $U, V$ têm interseção não vazia. Pode-se construir a função $H_{U, V}: h_{V}(U \cap$ $V) \rightarrow h_{U}(U \cap V)$ da forma seguinte

$$
H_{U, V}=h_{U} \circ h_{V}^{-1}
$$

a qual resulta ser um homeomorfismo de $h_{V}$ em $h_{U}$ e é uma das denominadas funções de transição que define uma mudança de coordenadas na interseção dos abertos. Figura A.3. Sejam

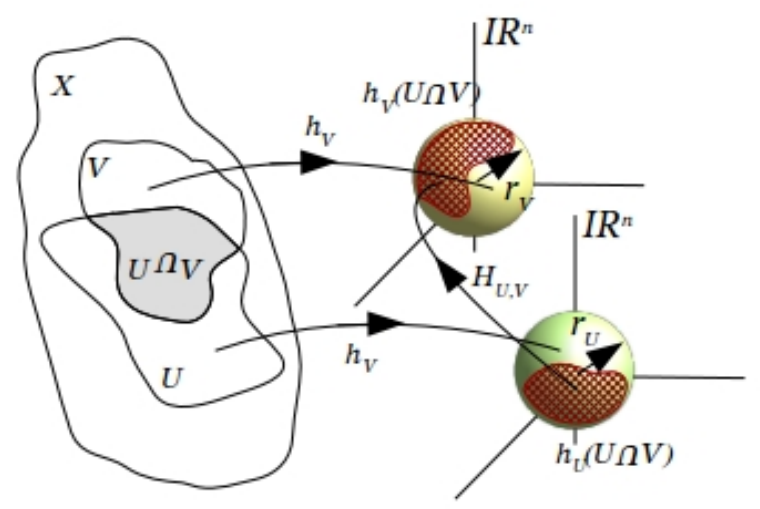

Figura A.3: Dois abertos euclidianos com interseção não vazia em $X$ mapeados por homeomorfismos em $\mathbb{R}^{n}$ permitem definir a função de transição $H_{U, V}$ homeomorfa. 
$A$ e $B$ dois conjuntos abertos homeomorfos em $\mathbb{R}^{n}$ com a métrica usual. Um homeomorfismo $f: A \rightarrow B$ é dito ser um difeomorfismo se $f$ e $f^{-1}$ forem diferenciáveis.

Seja uma variedade topológica segundo-contável $(X, \tau)$ de dimensão $n$. E sejam $\left(V, h_{V}\right)$ e $\left(U, h_{U}\right)$ cartas locais de coordenadas. Se diz que $\left(V, h_{V}\right)$ e $\left(U, h_{U}\right)$ são cartas compatíveis se

1. $V \cap U=\emptyset$, ou

2. $V \cap U \neq \emptyset$ mas a função de transição $H_{V, U}=h_{U} \circ h_{V}^{-1}$ for um difeomorfismo infinitamente diferenciável.

Seja uma variedade topológica segundo-contável $(X, \tau)$ de dimensão $n$. Um atlas $\mathcal{A}=\left\{\left(V_{\lambda}, h_{\lambda}\right)\right.$, $\left.\lambda \in \Lambda \mid X \subset \bigcup_{\lambda \in \Lambda} V_{\lambda}\right\}$ (com $\Lambda$ um conjunto indexado) é dito ser um atlas infinitamente diferenciável se todas suas cartas locais de coordenadas forem compatíveis.

Sejam $\mathcal{A}_{1}$ e $\mathcal{A}_{2}$ dois atlas infinitamente diferenciáveis. Se diz que $\mathcal{A}_{1}$ e $\mathcal{A}_{2}$ são compatíveis se $\mathcal{A}_{1} \cup \mathcal{A}_{2}$ for também um atlas infinitamente diferenciável. Decorre que existe uma relação de equivalência entre os atlas $\mathcal{A}_{1}$ e $\mathcal{A}_{2}$. Uma estrutura diferenciável $\mathcal{J}$ é uma classe de equivalência de atlas infinitamente diferenciáveis.

Uma variedade topológica segundo-contável de dimensão $n$ junto com uma estrutura diferenciável, ou seja, a tripla $(X, \tau, \mathcal{J})$ é dita ser uma variedade infinitamente diferenciável e as vezes denominada variedade diferencial. 



\section{Apêndice B}

\section{Mapas entre variedades e derivadas de Lie}

\section{B.1 Pullbacks e pushforwards}

Na seção 2.2.3, a ideia de recorrer ao conceito de espaço tangente em cada ponto $p$ de uma variedade $\mathcal{M}$ foi apresentada, em que os seus elementos chamados vetores podem ser entendidos como operadores diferenciais que agem sobre mapas $f \in \mathcal{C}^{\infty}$ de um espaço $\mathcal{F}(\mathcal{M})$ e definidos sobre a variedade, $f: \mathcal{M} \rightarrow \mathbb{R}$. Introduzimos agora os conceitos de pullback e pushforward, extraídos em boa parte de [25].

Sejam $\mathcal{M}$ e $\mathcal{N}$ duas variedades $(\operatorname{dim} \mathcal{M}=m, \operatorname{dim} \mathcal{N}=n), g: \mathcal{N} \rightarrow \mathbb{R}$ uma função, e seja $\phi: \mathcal{M} \rightarrow \mathcal{N}$ um mapa $\mathcal{C}^{\infty}$.

1. Se chama pullback da função $g$ por meio do mapa $\phi$ à função $\phi_{*} g: \mathcal{M} \rightarrow \mathbb{R}$ definida por meio da composição

$$
\phi_{*} g=g \circ \phi
$$

2. Se chama pushforward do vetor $\boldsymbol{V} \in T_{p} \mathcal{M}$ por meio do mapa $\phi$ ao vetor $\phi^{*} \boldsymbol{V} \in T_{\phi(p)} \mathcal{N}$ definido por meio da sua ação sobre funções de $\mathcal{F}(\mathcal{N})$,

$$
\phi^{*} \boldsymbol{V}(g)=\boldsymbol{V}\left(\phi_{*} g\right)=\boldsymbol{V}(g \circ \phi) .
$$

3. Se chama pullback do covetor $\boldsymbol{w} \in T_{\phi(p)}^{*} \mathcal{N}$ por meio do mapa $\phi$ ao covetor $\phi_{*} \boldsymbol{w} \in T_{p}^{*} \mathcal{M}$ definido por meio da sua ação sobre um vetor de $T_{p} \mathcal{M}$,

$$
\phi_{*} \boldsymbol{w}(\boldsymbol{V})=\boldsymbol{w}\left(\phi^{*} \boldsymbol{V}\right)
$$


4. Se chama pushforward do tensor tipo $(k, 0), \boldsymbol{K} \in T_{p} \mathcal{M} \otimes \underset{k-v e z e s}{ } \underset{\mathcal{M}}{ } \otimes T_{p} \mathcal{M}$ por meio do mapa $\phi$ ao tensor tipo $(k, 0), \phi^{*} \boldsymbol{K} \in T_{\phi(p)} \mathcal{N} \otimes T_{\phi(p)} \mathcal{N} \otimes \ldots \otimes T_{\phi(p)} \mathcal{N}$ definido por meio da sua ação sobre $k 1$-formas de $T_{\phi(p)}^{*} \mathcal{N}$

$$
\phi^{*} \boldsymbol{K}\left(\boldsymbol{w}_{1}, \boldsymbol{w}_{\mathbf{2}}, \ldots, \boldsymbol{w}_{\boldsymbol{k}}\right)=\boldsymbol{K}\left(\phi_{*} \boldsymbol{w}_{1}, \phi_{*} \boldsymbol{w}_{\mathbf{2}}, \ldots, \phi_{*} \boldsymbol{w}_{\boldsymbol{k}}\right)
$$

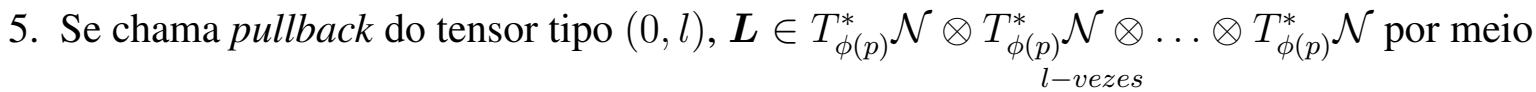
do mapa $\phi$ ao tensor tipo $(0, l), \phi_{*} \boldsymbol{L} \in T_{p}^{*} \mathcal{M} \otimes T_{p}^{*} \mathcal{M} \otimes \ldots \otimes T_{p}^{*} \mathcal{M}$ definido por meio da sua ação sobre $l$ vetores de $T_{p} \mathcal{M}$

$$
\phi_{*} \boldsymbol{L}\left(\boldsymbol{V}_{\mathbf{1}}, \boldsymbol{V}_{\mathbf{2}}, \ldots, \boldsymbol{V}_{\boldsymbol{l}}\right)=\boldsymbol{L}\left(\phi^{*} \boldsymbol{V}_{\mathbf{1}}, \phi^{*} \boldsymbol{V}_{\mathbf{2}}, \ldots, \phi^{*} \boldsymbol{V}_{\boldsymbol{l}}\right)
$$

Se o mapa $\phi$ for um difeomorfismo, ou seja, $\phi \in \mathcal{C}^{\infty}$ bijetor e sua inversa $\phi^{-1} \in \mathcal{C}^{\infty}$ [25] (cujo caso restringe a afirmação a variedades da mesma dimensão) pode-se estender a ação de $\phi^{*}$ para tensores gerais de tipo $(k, l)$.

6. A ação de $\phi^{*}$ sobre o campo tensorial ${ }^{1} \boldsymbol{T}$ tipo $(k, l)$, por meio do difeomorfismo $\phi$, é o tensor $^{2} \phi^{*} \boldsymbol{T}$ tipo $(k, l)$, definido por sua ação sobre $k 1$-formas de $T_{\phi(p)}^{*} \mathcal{N}$ e $l$ vetores de $T_{\phi(p)} \mathcal{N}$

$$
\phi^{*} \boldsymbol{T}\left(\boldsymbol{w}_{1}, \ldots, \boldsymbol{w}_{\boldsymbol{k}}, \boldsymbol{W}_{\mathbf{1}}, \ldots, \boldsymbol{W}_{\boldsymbol{l}}\right)=\boldsymbol{T}\left(\phi_{*} \boldsymbol{w}_{1}, \ldots, \phi_{*} \boldsymbol{w}_{\boldsymbol{k}}, \phi^{-1 *} \boldsymbol{W}_{\mathbf{1}}, \ldots, \phi^{-1 *} \boldsymbol{W}_{l}\right)
$$

Uma construção similar pode ser feita em termos de $\phi_{*}$, porém $\phi_{*}=\phi^{-1 *}$.

Se considerarmos agora o caso em que além de ser um difeomorfismo, $\phi: \mathcal{M} \rightarrow \mathcal{M}$, então existe a possibilidade de comparar o tensor original $\boldsymbol{T} \operatorname{com} \phi^{*} \boldsymbol{T}$. Se $\phi^{*} \boldsymbol{T}=\boldsymbol{T}, \phi$ é dito ser uma transformação de simetria para o campo tensorial $\boldsymbol{T}$. Se o campo tensorial $\boldsymbol{T}$ for o tensor métrico $\boldsymbol{g}$ a transformação de simetria $\phi$ é dita ser uma isometria.

\footnotetext{
${ }^{1} \boldsymbol{T} \in T_{p} \mathcal{M} \otimes \underset{k \text {-vezes }}{\otimes} T_{p} \mathcal{M} \otimes T_{p}^{*} \mathcal{M} \otimes \ldots \otimes T_{p}^{*} \mathcal{M}$

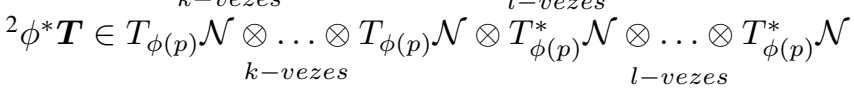




\section{B.2 Derivadas de Lie}

Seja o mapa $\phi_{t}: \mathbb{R} \times \mathcal{M} \rightarrow \mathcal{M}$ de forma que para cada valor de $t \in \mathbb{R}, \phi_{t}: \mathcal{M} \rightarrow \mathcal{M}$ é um difeomorfismo. Se define o produto entre os difeomorfismos $\phi_{t}$ e $\phi_{s}$ por meio da composição

$$
\phi_{t+s}=\phi_{t} \circ \phi_{s}, \forall t, s \in \mathbb{R}
$$

Segue que se $\phi_{t=0}=\phi_{0}, \log 0 \phi_{s}=\phi_{0} \circ \phi_{s}$ e $\phi_{0}$ seria o mapa identidade sob o produto (B.7). Pode-se mostrar que com essa operação, o conjunto dos $\phi_{t}$ é um grupo, chamado grupo de difeomorfismos de um parâmetro.

Por outro lado, fixando um ponto na variedade $\mathcal{M}$, por exemplo o ponto $p$, o mapa $\phi_{t}(p)$ : $\mathbb{R} \rightarrow \mathcal{M}$, define uma curva que passa no ponto $p$ em $t=0$. Seja $\boldsymbol{V}_{\boldsymbol{p}}$ o vetor tangente à curva em $p$ (ou em $t=0$ ), então associado com o grupo de um parâmetro está o campo vetorial $\boldsymbol{V}$ de vetores tangentes. Mesmo acontece no sentido contrário. Seja $\boldsymbol{V}$ um campo vetorial suave definido sobre $\mathcal{M}$, com $\boldsymbol{V} \neq 0 \forall p \in \mathcal{M}$, podemos achar curvas integrais do campo de forma que uma e só uma curva passe no ponto $p$. Logo, para cada ponto $p \in \mathcal{M}$ se define $\phi_{t}(p)$, o ponto com parâmetro $t$ ao longo da curva integral de $\boldsymbol{V}$ que começa em $p$. O campo vetorial $\boldsymbol{V}$ é conhecido como gerador do difeomorfismo.

Se define a derivada de Lie do campo tensorial $\boldsymbol{T}$ ao longo do campo vetorial $\boldsymbol{V}$ como sendo [25]

$$
\mathcal{L}_{\boldsymbol{V}} \boldsymbol{T}=\lim _{t \rightarrow 0} \frac{\left(\phi_{-t}^{*} \boldsymbol{T}\right)(p)-\boldsymbol{T}(p)}{t}
$$

o que significa pegar o tensor $\boldsymbol{T}$ no ponto $p$ e compará-lo com o mesmo tensor no ponto $\phi_{t}(p)$ trazido de volta no ponto $p$ por meio do pushforward $\phi_{-t}^{*}$. A derivada de Lie é um mapa linear que cumpre com a regra de Leibnitz do produto. Da própria definição e fazendo uso de uma carta local, é possível mostrar que

1. A derivada de Lie de uma função $f \in \mathcal{F}$ é

$$
\mathcal{L}_{V} f=V^{\mu} \partial_{\mu}(f) .
$$

2. A derivada de Lie de um vetor pode-se expressar em termos de um comutador (2.21),

$$
\mathcal{L}_{\boldsymbol{V}} U^{\mu}=[\boldsymbol{V}, \boldsymbol{U}]^{\mu}=V^{\nu} \nabla_{\nu} U^{\mu}-U^{\nu} \nabla_{\nu} V^{\mu}
$$


3. A derivada de Lie de um covetor pode ser construida a partir da relação anterior

$$
\mathcal{L}_{V} w_{\mu}=w_{\nu} \nabla_{\mu} V^{\nu}+V^{\nu} \nabla_{\nu} w_{\mu}
$$

4. A derivada de Lie de um campo tensorial geral pode ser construída, mas a dos tensores tipo $(0,2)$ é de nosso particular interesse no capítulo 3 , e se expressa como

$$
\mathcal{L}_{V} S_{\mu \nu}=V^{\sigma} \nabla_{\sigma} S_{\mu \nu}+S_{\sigma \nu} \nabla_{\mu} V^{\sigma}+S_{\mu \sigma} \nabla_{\nu} V^{\sigma}
$$




\section{Apêndice C}

\section{McVittie generalizado e as equações de campo de Einstein}

As equações de campo de Einstein com constante cosmológica dadas pela expressão (2.64), podem ser expressas também como

$$
G_{\mu \nu}=8 \pi T_{\mu \nu}^{\prime}
$$

onde foi usado o tensor de Einstein $G_{\mu \nu}=R_{\mu \nu}-R g_{\mu \nu} / 2$, e onde a parte com constante cosmológica foi absorbida pelo tensor de energia-momento denominado agora $T_{\mu \nu}^{\prime}$. A substituição do elemento de linha de McVittie generalizado na forma (4.25) no lado esquerdo de (C.1) e a substituição do tensor de energia-momento de fluido imperfeito do lado direito produz as seguintes equações diferentes de zero [20]

$$
\begin{gathered}
G^{t}{ }_{t}=-3\left[\frac{\dot{a}}{a}+\frac{2 \dot{m}}{2 a r-m}\right]^{2}=-8 \pi \rho=8 \pi T^{\prime t}{ }_{t} \\
G_{r}^{t}=-8 a \dot{m} \frac{2 a r+m}{(2 a r-m)^{3}}=-8 \pi \chi\left[\left(\frac{2 a r+m}{2 a r-m}\right) \partial_{r} T+\frac{4 m a T}{(2 a r-m)^{2}}\right]=8 \pi T^{\prime t}{ }_{r}, \\
G^{i}{ }_{i}=G^{t}{ }_{t}-2 \frac{2 a r+m}{2 a r-m} \frac{d}{d t} \sqrt{-\frac{G^{t} t}{3}}=8 \pi p-24 \pi \zeta\left(\frac{\dot{a}}{a}+\frac{2 \dot{m}}{2 a r-m}\right)=8 \pi T^{\prime i}{ }_{i}
\end{gathered}
$$

Na equação (C.4) não está sendo usada a convenção de índices repetidos, a notação $G_{i}^{i}$ e $T^{\prime i}{ }_{i}$ apenas representam elementos na diagonal no tensor de Einstein e no tensor de energia momento, e os valores de $i$ são $i=r, \theta, \phi$. O conjunto de expressões anteriores representam 
vínculos na escolha das funções $a(t), m(t)$, assim como da pressão e densidade do fluido e os coeficientes associados com o fluido imperfeito (4.26). 


\section{Referências Bibliográficas}

[1] F. W. Dyson, A. S. Eddington, and C. Davidson. A Determination of the Deflection of Light by the Sun's Gravitational Field, from Observations Made at the Total Eclipse of May 29, 1919. Philosophical Transactions of the Royal Society of London Series A, 220:291-333, 1920.

[2] Abbott, B. P. et al. Observation of gravitational waves from a binary black hole merger. Phys. Rev. Lett., 116:061102, Feb 2016.

[3] GRAVITY Collaboration et al. Detection of the gravitational redshift in the orbit of the star s2 near the galactic centre massive black hole. A\&A, 615:L15, 2018.

[4] S. Dodelson. Modern Cosmology. Elsevier Science, 2003.

[5] L. Amendola and S. Tsujikawa. Dark Energy: Theory and Observations. Cambridge University Press, 2010.

[6] Sébastien Clesse and Juan García-Bellido. Massive primordial black holes from hybrid inflation as dark matter and the seeds of galaxies. Phys. Rev. D, 92:023524, Jul 2015.

[7] A. Kashlinsky. Ligo gravitational wave detection, primordial black holes, and the near-ir cosmic infrared background anisotropies. The Astrophysical Journal Letters, 823(2):L25, 2016.

[8] Bernard Carr, Florian Kühnel, and Marit Sandstad. Primordial black holes as dark matter. Phys. Rev. D, 94:083504, Oct 2016.

[9] A. D. Dolgov. Primordial Black Holes and Cosmological Problems. In 18th Lomonosov Conference on Elementary Particle Physics Moscow, Russia, August 24-30, 2017, 2017.

[10] Friedrich Kottler. über die physikalischen grundlagen der einsteinschen gravitationstheorie. Annalen der Physik, 361(14):401-462.

[11] G. C. McVittie. The mass-particle in an expanding universe. Mon. Not. Roy. Astron. Soc., 93:325-339, 1933.

[12] Kayll Lake and Majd Abdelqader. More on McVittie's Legacy: A Schwarzschild - de Sitter black and white hole embedded in an asymptotically $\Lambda$ CDM cosmology. Phys. Rev., D84:044045, 2011.

[13] Brien C. Nolan. A Point mass in an isotropic universe: Existence, uniqueness and basic properties. Phys. Rev., D58:064006, 1998. 
[14] B. C. Nolan. A Point mass in an isotropic universe. 2. Global properties. Class. Quant. Grav., 16:1227-1254, 1999.

[15] Brien C. Nolan. A Point mass in an isotropic universe. 3. The region R less than or $=$ to 2m. Class. Quant. Grav., 16:3183-3191, 1999.

[16] Valerio Faraoni, Changjun Gao, Xuelei Chen, and You-Gen Shen. What is the fate of a black hole embedded in an expanding universe? Phys. Lett., B671:7-9, 2009.

[17] Nemanja Kaloper, Matthew Kleban, and Damien Martin. McVittie's Legacy: Black Holes in an Expanding Universe. Phys. Rev., D81:104044, 2010.

[18] Valerio Faraoni, Andres F. Zambrano Moreno, and Angus Prain. The charged McVittie spacetime. Phys. Rev., D89(10):103514, 2014.

[19] Valerio Faraoni and Audrey Jacques. Cosmological expansion and local physics. Phys. Rev., D76:063510, 2007.

[20] Daniel C. Guariento, Michele Fontanini, A. M. da Silva, and Elcio Abdalla. Realistic fluids as source for dynamically accreting black holes in a cosmological background. Phys. Rev., D86:124020, 2012.

[21] Matthias Blau. Lecture Notes on General Relativity. Albert Einstein Center for fundamental physics, Institut für Theoretische Physik, Universität Bern, 2016. Disponível em: http://www.blau.itp.unibe.ch/GRLecturenotes.html.

[22] E. Poisson. A Relativist's Toolkit: The Mathematics of Black-Hole Mechanics. Cambridge University Press, 2004.

[23] Ivan Booth. Black-hole boundaries. Canadian Journal of Physics, 83(11):1073-1099, 2005.

[24] S.M. Carroll. Spacetime and Geometry: An Introduction to General Relativity. Addison Wesley, 2004.

[25] R.M. Wald. General Relativity. University of Chicago Press, 1984.

[26] M. Capek. The Concepts of Space and Time: Their Structure and Their Development. Boston Studies in the Philosophy and History of Science. Springer Netherlands, 2014.

[27] R. Penrose and J.G. Sanz. El camino a la realidad: una guía completa de las leyes del universo. Random House Mondadori, 2006.

[28] Albert Einstein. On the electrodynamics of moving bodies. Annalen Phys., 17:891-921, 1905. [Annalen Phys.14,194(2005)].

[29] D. DeWitt and B. DeWitt. Lectures delivered at Les Houches during the 1963 session of the summer school of theoretical physics, University of Grenoble. Relativity, groups and topology. New York, 1964.

[30] S. Weinberg. Gravitation and cosmology: principles and applications of the general theory of relativity. Wiley, 1972. 
[31] J. G. Pereira R. Aldrovandi. Notes for a course on classical fields. Instituto de Física Teórica, Universidade Estadual Paulista, 2008.

[32] W. Israel. DARK STARS: THE EVOLUTION OF AN IDEA. 1987.

[33] J.B. Griffiths and J. Podolskỳ. Exact Space-Times in Einstein's General Relativity. Cambridge Monographs on Mathematical Physics. Cambridge University Press, 2009.

[34] R. D’Inverno. Introducing Einstein's Relativity. Clarendon Press, 1992.

[35] S.W. Hawking and G.F.R. Ellis. The Large Scale Structure of Space-Time. Cambridge Monographs on Mathem. Cambridge University Press, 1973.

[36] S.W.H. W. Israel, S.W. Hawking, W. Israel, W.I. Israel, and A. Einstein. General Relativity; an Einstein Centenary Survey. Cambridge University Press, 1979.

[37] Valerio Faraoni. Cosmological and Black Hole Apparent Horizons. Lect. Notes Phys., 907:pp.1-199, 2015.

[38] S.A. Hayward. General laws of black hole dynamics. Phys.Rev., D49:6467-6474, 1994.

[39] Pau Figueras, Veronika E. Hubeny, Mukund Rangamani, and Simon F. Ross. Dynamical black holes and expanding plasmas. Journal of High Energy Physics, 2009(04):137, 2009.

[40] María del Prado Martín Moruno. Cosmologá no estándar: presente y futuro del Universo. PhD thesis, Universidad Autónoma de Madrid. Departamento de Física Teórica, 2010.

[41] Jorge L. Cervantes-Cota and George Smoot. Cosmology today-A brief review. AIP Conf. Proc., 1396:28-52, 2011.

[42] C.W. Misner, K.S. Thorne, and J.A. Wheeler. Gravitation. Number pt. 3 in Gravitation. W. H. Freeman, 1973.

[43] Planck Collaboration et al. Planck 2013 results. xvi. cosmological parameters. A\&A, 571:A16, 2014.

[44] Matteo Carrera and Domenico Giulini. Influence of global cosmological expansion on local dynamics and kinematics. Rev. Mod. Phys., 82:169-208, Jan 2010.

[45] Valerio Faraoni. Evolving black hole horizons in General Relativity and alternative gravity. Galaxies, 1(3):114-179, 2013.

[46] Olivier Lennon, John March-Russell, Rudin Petrossian-Byrne, and Hannah Tillim. Black hole genesis of dark matter. Journal of Cosmology and Astroparticle Physics, 2018(04):009, 2018.

[47] João C. A. Barata. Notas para um curso de física-matemática. Departamento de física matemática, Instituto de Física, Universidade de São Paulo, 2018. Disponível em: http: // denebola.if.usp.br/ jbarata/Notas_de_aula/capitulos.html. 